\title{
Pauli-Jordan function and scalar field quantization in $\kappa$-Minkowski noncommutative spacetime
}

\author{
Flavio Mercati ${ }^{*}$ and Matteo Sergola ${ }^{\dagger}$ \\ Dipartimento di Fisica, Sapienza Università di Roma, P.le A. Moro 2, 00185 Roma, Italy
}

(Received 21 June 2018; published 22 August 2018)

\begin{abstract}
We study a complex free scalar field theory on a noncommutative background spacetime called $\kappa$-Minkowski. We ensure the Lorentz invariance of the theory by assuming an "elliptic de Sitter" topology for momentum space. To define covariant quantization rules, we introduce a noncommutative Pauli-Jordan function, which is invariant under $\kappa$-deformed Poincaré transformations, as well as diffeomorphisms of momentum space. We derive the algebra of creation and annihilation operators implied by our Pauli-Jordan function. Finally, we use our construction to study the structure of the light cone in $\kappa$-Minkowski spacetime, and to derive its physical consequences.
\end{abstract}

DOI: 10.1103/PhysRevD.98.045017

\section{INTRODUCTION}

Quantum field theory (QFT) on Minkowski spacetime is arguably the most successful paradigm in physics, both for the precision with which some of its predictions have been tested, and for the variety of phenomena it is capable to describe. As a description of Nature, flat-space QFT is clearly an effective theory, as it ignores the dynamics of the gravitational field, for which we currently have no satisfying quantum formulation. The unspoken underlying assumption is that, whatever the correct quantum theory of gravity is, it will admit a "ground state" which looks like Minkowski spacetime. Then flat-space QFT would be a good description of matter as long as graviton production can be ignored. This last condition is a safe assumption in the experimental regimes we have access to. This is because the coupling between matter and gravity is controlled by the Planck scale $E_{p} \sim 10^{28} \mathrm{eV}$, which is an enormous energy scale that makes for an extremely small coupling constant. Our current understanding of quantum gravity suggests that the aforementioned assumption might not be correct. The ground state of general relativity might be something different from Minkowski space, which only looks like it in the low-energy limit. The strongest indications come from $2+1$ dimensional quantum gravity, which, because it lacks local propagating degrees of freedom (gravitons), can be quantized with topological QFT

\footnotetext{
flavio.mercati@gmail.com

†matteo.sergola@gmail.com
}

Published by the American Physical Society under the terms of the Creative Commons Attribution 4.0 International license. Further distribution of this work must maintain attribution to the author(s) and the published article's title, journal citation, and DOI. Funded by SCOAP ${ }^{3}$. methods. Coupling this theory to matter and integrating away the gravitational degrees of freedom, one ends up with a nonlocal effective theory $[1,2]$. This theory admits a description as a QFT on a noncommutative background, in the sense that the ordinary algebra of functions on spacetime [which is an Abelian algebra when endowed with the pointwise product $(f \cdot g)(x)=f(x) g(x)]$ is replaced with a noncommutative algebra. The Planck scale (or rather its inverse, which in $\hbar=c=1$ units is the Planck length $L_{p} \sim 10^{-35} \mathrm{~m}$ ) plays the role of noncommutativity parameter, similar to that played by $\hbar$ in ordinary quantum mechanics. In particular it appears on the right-hand side of uncertainty relations between coordinate functions $x^{\mu}$, and therefore there is a sense in which the noncommutative geometry described by this algebra should look like the commutative geometry of Minkowski spacetime in the large-scale/infrared limit. Let us restate this important point: the "background state" of $2+1 \mathrm{D}$ quantum gravity coupled with matter is not QFT on Minkowski spacetime. It is rather a QFT on a noncommutative geometry which reduces to Minkowski space only in the low-energy limit.

In light of the lesson of $2+1 \mathrm{D}$ quantum gravity, studying noncommutative geometries in $3+1 \mathrm{D}$, and the QFTs that can be built upon them, acquires a great interest $[3,4]$. Because of our lack of understanding of $3+1 \mathrm{D}$ quantum gravity, we presently have no way to repeat the exercise done in $2+1 \mathrm{D}$ of integrating away the gravitational field to uncover the correct effective theory of matter on a quantum-gravity background. For this reason, we are compelled to study all the possible $3+1 \mathrm{D}$ noncommutative geometries whose noncommutativity parameter depends on the Planck length. If we are able to develop consistent QFTs on such backgrounds, there is a chance that their phenomenological implications could 
be experimentally tested, giving us precious hints towards the correct quantum theory of gravity in $3+1 \mathrm{D}$.

A powerful way to describe a geometry is through its symmetries. Minkowski spacetime, e.g., is completely characterized by the fact that it is a maximally symmetric space which is flat. The first condition (maximal symmetry) reduces the possible choices to only three: de Sitter (for positive curvature), anti-de Sitter (negative curvature), and Minkowski (flat). Then the spacetime can be understood as the quotient of a 10-dimensional Lie group [(A)-dS or Poincaré, depending on the curvature] by a 6-dimensional isotropy subgroup (the Lorentz group). Interestingly, these structures generalize to noncommutative spaces. Lie groups are generalized to something called quantum groups $[5,6]$ : essentially, the algebra of functions on the group manifold becomes noncommutative (see next section).

Remarkably, there is only a limited number of quantum groups that reduce to the Poincare group when the noncommutativity parameter vanishes [7]. In particular, if we require the noncommutativity parameter to depend linearly on the Planck scale, and to admit a regular limit as the cosmological constant is sent to zero, we are left with (almost) only one choice [8]. This is the $\kappa$-Poincaré quantum group $[9,10]$, where $\kappa$ refers to the (inverse of the) noncommutativity parameter, which has the dimensions of an energy. Field theories that are invariant under $\kappa$ Poincaré symmetries have been studied extensively in the past (see for instance [11-30]), especially at the classical level (i.e., in the limit $\hbar \rightarrow 0$ while keeping $\kappa$ finite). However, the understanding of $\kappa$-deformed field theories is still far from complete, especially for what regards their second quantization.

In the present work we discuss a new strategy for the construction and quantization of a complex scalar field on $\kappa$-Minkowski. Our approach is explicitly $\kappa$-Poincaré covariant, and independent on the choice of basis of the spacetime symmetry algebra (an important feature which most previous approaches lacked; see below). A key element of our analysis is the observation that, in order to preserve Lorentz invariance, the momentum space of fields on $\kappa$-Minkowski must have the topology of elliptic de Sitter space. It has in fact been observed long ago [31] that the $\kappa$-deformed momentum space has a de Sitter geometry. However, at the global level the momentum space covers only half of the de Sitter hyperboloid, and that half is not closed under the action of Lorentz transformations. The Lorentz orbits become complete (i.e., do not terminate at a finite boundary) only if we assume the elliptic topology $d S / \mathbb{Z}^{2}$ for the de Sitter hyperboloid (see Sec. II).

Our approach will allow us to write the algebra of creation and annihilation operators for a scalar filed, which in turn defines the Fock space of the theory. An important feature of our analysis is that we define the quantization rules of the theory in a covariant way, using the PauliJordan function. This affords us to avoid using canonical quantization and therefore Hamiltonian formulations. Such formulations are problematic, because $\kappa$-Minkowski (the noncommutative spacetime whose symmetries are described by the $\kappa$-Poincare group) does not admit the notion of constant-time slices. ${ }^{1}$

Our covariant approach has an additional bonus: it gives us a generalization of the notion of light cone. In fact the commutative Pauli-Jordan function vanishes outside the light cone: it measures the commutator between quantum fields at different points, and such a commutator must vanish on spacelike-separated points in order for causality to be respected. In the noncommutative case the PauliJordan function turns into an element of a noncommutative algebra, and therefore some more work is necessary in order to extract a notion of light cone from it. Our proposal is to interpret the Pauli-Jordan function as an operator on a Hilbert space of "geometrical" states, i.e., states of the background quantum geometry. By calculating the expectation value of said operator on a state that is peaked around a classical pair of points (what we call semiclassical state) we are able to extract the dependence of the Pauli-Jordan function on the coordinates of the classical points around which our state is peaked. It turns out that this dependence is not confined inside the classical light cone, but it rather "spills out" within a region whose size is the geometric mean between the Planck length and the distance from the origin of the light cone. The derivation of these results is the subject of Sec. V.

Our calculation of the Pauli-Jordan function allows us to intervene in a debate that has been ongoing since the early days of $\kappa$-Poincaré. This is whether the deformations of relativistic kinematics predicted by $\kappa$-Poincaré imply anomalous in-vacuum dispersion and whether such dispersion can be detected with present-day technology. At the turn of the century it was proposed that the Planck scale might enter new physics in a fashion similar to that of the speed of light. $c$ in fact is a speed constant that takes the same value for all inertial observers, and in order to accommodate the relativistic invariance of $c$ one needs to deform Galilean relativity in a $c$-dependent way. Similarly, as suggested in [32-34], $E_{p}$ could be a new energy scale that appears the same to all inertial observers, but this requires a deformation of special relativity into a new relativistic theory with two invariant scales (this proposal was dubbed doubly special relativity [32-37]). Soon after this idea was proposed, $\kappa$-Poincaré was identified as a candidate model to realize such two-scale generalization of special relativity [38]. Indeed, as we show in Eq. (28) below, one finds deformed Lorentz transformations laws for energy and momentum which depend on both $c$ and the Planck scale. $\kappa$-Poincare is still

\footnotetext{
${ }^{1}$ The commutation relations of $\kappa$-Minkowski coordinates are such that a sharply defined time coordinate implies infinite uncertainty on the spatial coordinates (see Sec. IV).
} 
today the best candidate for a consistent model realizing the idea of doubly special relativity. Unfortunately introducing the Planck scale as an observer-independent constant makes it harder to detect its effects. In fact, most meaningful constraints for a possible role of $E_{p}$ in physics come from testing some conjectured Lorentz-breaking effects, whereupon Michelson-Morley-like experiments putting constraints on a "quantum-gravity aether" are easy to devise and extremely powerful [39-42]. If the Planck scale enters as a deformation of some laws of physics in a way that does not depend on the inertial frame, it becomes much harder to detect. One of the best proposals for such a test is in-vacuum dispersion: if the relativistic kinematics is deformed in a Planck-scale-dependent way, it is conceivable that the energy-momentum dispersion relation acquires Planck-scale corrections. Then the group velocity of particles propagating in a vacuum should get Planckscale corrections, and it is just a matter of dimensional analysis to show which kind of dependences on energy and momentum such a deformation could acquire [43]. One possibility is that the dispersion is such that distant pointlike sources arrive on Earth with a time delay of the form

$$
\delta t \propto L_{p} E L,
$$

where $\delta t$ might be a systematic delay of all particles or a random uncertainty on the time of arrival, different for each particle. $E$ is the energy of the particle and $L$ is the distance of the source (in units $\hbar=c=1$ ). Such a law can be meaningfully tested with present-day observations: in particular gamma ray bursts provide bright sources of photons of energies up to $1 \mathrm{TeV}$ at distances of the order of a billion light years. With such numbers the law (1) predicts time delays of the order of $1 \mathrm{sec}$. Indeed the FermiLAT experiment has been testing such a hypothesis for years, putting constraint on the energy scale of the proposed quantum-gravity effect [44] of the order of $E_{p}$ or more. More recently, a proposal to test the hypothesis that the high-energy neutrinos observed by the IceCube observatory are originated in gamma ray bursts, and are subject to the same proposed quantum-gravity effects gained significant attention [45]. Similarly, the coincidence between LIGO/VIRGO detections of gravitational waves with electromagnetic counterparts can represent powerful independent tests of Planck-scale-originated time delays of different particles [46]. Other tests have been proposed, e.g., based on primordial cosmology [47]. So, soon after a law like (1) was first proposed, it was conjectured that it would be a prediction of $\kappa$-Poincaré, ${ }^{2}$ and then gamma ray

\footnotetext{
${ }^{2}$ However, $\kappa$-Poincaré is not the only theoretical scenario that was proposed to be relevant for this kind of phenomenology: models inspired by string theory [48-52], loop quantum gravity $[53,54]$, and in general, Lorentz-violating extensions of known physics $[39,41]$ are constrained by it.
}

bursts would be the perfect arena to test the predictions of this model. In the present paper we study the constraints imposed by $\kappa$-Poincaré-invariant QFT on the causal structure of $\kappa$-Minkowski. This will allow us to establish with a higher level of confidence whether gamma ray burst phenomenology is able to put meaningful bounds on our model.

In the Sec. II we briefly review the mathematical tools needed for our analysis, and give a brief recap of the things we already know about scalar fields and the geometry of momentum space. In Sec. III we generalize the KleinGordon equation to $\kappa$-Minkowski and in Sec. IV we introduce the " $\kappa$-deformed" Pauli-Jordan function. In Sec. V we use the Pauli-Jordan function to study the noncommutative light cone of our theory and in Sec. VI we present future perspectives and conclusions.

\section{THE $\kappa$-POINCARÉ QUANTUM GROUP AND THE $\kappa$-MINKOWSKI SPACETIME}

\section{A. The standard Poincaré group as a Hopf algebra}

The structure of the Poincaré group $\mathcal{G}$ can be described in algebraic terms by considering the algebra of complexvalued functions on the group $\mathbb{C}[\mathcal{G}]$, and by introducing three maps which are dual ${ }^{3}$ to the three defining axioms of Lie groups. First one needs to introduce coordinate functions on the group $\Lambda^{\mu}{ }_{\nu}, a^{\mu}: \mathcal{G} \rightarrow \mathbb{C}$, i.e., elements of $\mathbb{C}[\mathcal{G}]$ which associate with each group element $g \in \mathcal{G}$ its coordinates in the standard representation of the group.

The group product can be described by a coproduct map $\Delta: \mathbb{C}[\mathcal{G}] \rightarrow \mathbb{C}[\mathcal{G}] \otimes \mathbb{C}[\mathcal{G}]:$

$\Delta\left[\Lambda^{\mu}{ }_{\nu}\right]=\Lambda_{\rho}^{\mu} \otimes \Lambda^{\rho}{ }_{\nu}, \quad \Delta\left[a^{\mu}\right]=a^{\mu} \otimes \mathbb{1}+\Lambda^{\mu}{ }_{\nu} \otimes a^{\nu}$.

$\Delta\left[\Lambda^{\mu}{ }_{\nu}\right]$ is now a function that associates with two group elements $g, h \in \mathcal{G}$ the coordinates, in the coordinate system $\Lambda^{\mu}{ }_{\nu}, a^{\mu}$, of the product element $g \cdot h: \Delta\left[\Lambda^{\mu}{ }_{\nu}\right](g, h)=$ $\Lambda^{\mu}{ }_{\nu}(g) \Lambda^{\nu}{ }_{\mu}(h)=\Lambda^{\mu}{ }_{\nu}(g \cdot h)$, and similarly for $\Delta\left[a^{\mu}\right]$ : $\Delta\left[a^{\mu}\right](g, h)=a^{\mu}(g)+\Lambda_{\nu}^{\mu}(g) a^{\nu}(h)=a^{\mu}(g \cdot h)$.

The group inverse can be encoded into an antipode map $S: \mathbb{C}[\mathcal{G}] \rightarrow \mathbb{C}[\mathcal{G}]:$

$$
S\left[\Lambda^{\mu}{ }_{\nu}\right]=\left(\Lambda^{-1}\right)^{\mu}{ }_{\nu}, \quad S\left[a^{\mu}\right]=-\left(\Lambda^{-1}\right)^{\mu}{ }_{\nu} a^{\nu},
$$

which, when calculated on the coordinate functions give two functions whose value on a group element $g$ is the coordinates of the inverse element $g^{-1}: S\left[\Lambda^{\mu}{ }_{\nu}\right](g)=$ $\left(\Lambda^{-1}\right)^{\mu}{ }_{\nu}(g)=\Lambda_{\nu}^{\mu}{ }_{\nu}\left(g^{-1}\right), S\left[a^{\mu}\right](g)=-\Lambda^{\mu}{ }_{\nu} a^{\nu}(g)=a^{\mu}\left(g^{-1}\right)$.

Finally, the map that stands in for the unit is called counit $\varepsilon: \mathbb{C}[\mathcal{G}] \rightarrow \mathbb{C}:$

$$
\varepsilon\left[\Lambda^{\mu}{ }_{\nu}\right]=\delta_{\nu}^{\mu}, \quad \varepsilon\left[a^{\mu}\right]=0
$$

\footnotetext{
${ }^{3}$ In the category-theory sense of inverting all the arrows.
} 
where $\varepsilon\left[\Lambda^{\mu}{ }_{\nu}\right]=\Lambda_{\nu}^{\mu}(e)$ and $\varepsilon\left[a^{\mu}\right]=a^{\mu}(e)$ give the coordinates of the identity element $e \in \mathcal{G}$ in the coordinate system $\Lambda_{\nu}^{\mu}, a^{\mu}$.

In relation to the structures of the commutative algebra $\mathbb{C}[\mathcal{G}]$ (product and linear combination), the maps $\Delta$ and $\varepsilon$ are algebra homomorphisms: $\Delta[f g]=\Delta[f] \Delta[g], \Delta[f+g]=$ $\Delta[f]+\Delta[g]$, while $S$ is an antihomomorphism.

With the three maps $\Delta, S$, and $\varepsilon$ we can completely describe the group, provided that they satisfy a compatibility axiom:

$$
\mu \circ(S \otimes \mathrm{id}) \circ \Delta=\mu \circ(\mathrm{id} \otimes S) \circ \Delta=\mathbb{1} \varepsilon,
$$

where $\mu: \mathbb{C}[\mathcal{G}] \otimes \mathbb{C}[\mathcal{G}] \rightarrow \mathbb{C}[\mathcal{G}]$ is the product of $\mathbb{C}[\mathcal{G}]$ (the pointwise product between functions) and $i d$ is the identity map. This axiom coincides with the definition of the inverse $g^{-1} \cdot g=g \cdot g^{-1}=e$.

\section{B. Generalization to quantum groups}

Having achieved this unusual description of the wellknown Poincaré group, we can disclose now the reason for going through all this trouble. The commutative algebra of functions $\mathbb{C}[\mathcal{G}]$ can be replaced with a non-Abelian algebra $\mathbb{C}_{\kappa}[\mathcal{G}]$, whose product now does not admit anymore the interpretation of pointwise product between functions, nor its elements the interpretation of functions on $\mathcal{G}$. We are now dealing with a quantum group [6,55], whose algebra of functions is noncommutative. In the case of the Poincare group in $3+1$ dimensions, there appears to be essentially only a seven-parameter space of candidates which admit the interpretation of the flat-space limit of a Planck-scale deformation (first order in the Planck length) of the Poincaré group [8]. If three of these parameters (those associated with a "Reshetikhin twist" [8]) are set to zero, we obtain the $\kappa$-Poincaré group, defined by the commutation relations [9]

$$
\begin{aligned}
{\left[a^{\mu}, a^{\nu}\right] } & =i\left(v^{\mu} a^{\nu}-v^{\nu} a^{\mu}\right), \quad\left[\Lambda_{\nu}^{\mu}, \Lambda^{\rho}{ }_{\sigma}\right]=0, \\
{\left[\Lambda_{\nu}^{\mu}, a^{\rho}\right] } & =-i\left[\left(\Lambda^{\mu}{ }_{\sigma} v^{\sigma}-v^{\mu}\right) \Lambda_{\nu}^{\rho}+\left(\Lambda_{\nu}{ }_{\nu} v_{\sigma}-v_{\nu}\right) \eta^{\mu \rho}\right],
\end{aligned}
$$

where $\eta_{\mu \nu}=\operatorname{diag}(-,+,+,+)$ and $v^{\mu}=\left(v^{0}, v^{1}, v^{2}, v^{3}\right)$ are four deformation parameters, which should be of the order of the Planck length. In a mathematical sense, all choices of $v^{\mu}$ with the same sign of the norm, $v_{\mu} v^{\mu}$, are equivalent (they are related by algebra automorphisms). Then the mathematically inequivalent cases are only three: when $v^{\mu}$ is spacelike, lightlike, or timelike [13]. Mathematical equivalence aside, the question whether different choices of $v^{\mu}$ lead to different physics remains open, and we do not intend to dwell on these issues in the present paper. For now, it is sufficient to study one particular case, and we choose the most-studied one, which is $v^{\mu}$ timelike and of the form

$$
v^{\mu}=\frac{1}{\kappa} \delta_{0}^{\mu}
$$

where $\kappa$ is the eponymous parameter of the $\kappa$-Poincare group. $\kappa$ has the dimensions of an energy, and it is expected to be close to the Planck energy, if the $\kappa$-Poincare group is to describe deformations originated in a presently unknown quantum theory of gravity.

Notice that the commutation relations (6) admit two subalgebras, one generated by the four translation generators $a^{\mu}$ and another one generated by the Lorentz matrices $\Lambda^{\mu}{ }_{\nu}$ (moreover the latter algebra is commutative; therefore, the Lorentz subgroup $S O(3,1)$ is classical). The fact that the translation generators close a subalgebra is a consequence of the fact that the $\kappa$-Poincaré algebra is coisotropic with respect to Lorentz transformations [56]. This is required to be able to talk about a quantum homogeneous space, generated by quotienting $\mathbb{C}_{K}[\mathcal{G}]$ by the Lorentz subgroup $\mathcal{A}=\mathbb{C}_{\kappa}[\mathcal{G}] / S O(3,1)$. This is a noncommutative algebra generated by

$$
\left[x^{\mu}, x^{\nu}\right]=i\left(v^{\mu} x^{\nu}-v^{\nu} x^{\mu}\right)
$$

or, in the timelike case we are concerned with in the present paper,

$$
\left[x^{0}, x^{i}\right]=\frac{i}{\kappa} x^{i}, \quad\left[x^{i}, x^{j}\right]=0
$$

The $x^{\mu}$ generators should be interpreted as coordinate functions on a noncommutative spacetime, which we call $\kappa$ Minkowski. The algebra $\mathcal{A}$ is to be interpreted as the algebra of functions on $\kappa$-Minkowski, or, which is the same thing, the algebra of scalar fields. $\mathcal{A}$ is obtained by taking all possible (finite or infinite) products and sums of $x^{\mu}$, i.e., $\sum_{n=0}^{\infty} c_{\mu_{1} \ldots \mu_{n}} x^{\mu_{1}} \ldots x^{\mu_{n}}$ with $c_{\mu_{1} \ldots \mu_{n}} \in \mathbb{C}$.

Just like commutative homogeneous spaces, $\kappa$-Minkowski comes equipped with an action of the $\kappa$-Poincaré group which leaves it invariant. In the Hopf algebra language, as could be expected, this is expressed by means of a (left-) coaction map, $\Delta_{L}: \mathcal{A} \rightarrow \mathbb{C}[\mathcal{G}] \otimes \mathcal{A}$,

$$
\Delta_{L}\left[x^{\mu}\right]=\Lambda_{\nu}^{\mu} \otimes x^{\nu}+a^{\mu} \otimes \mathbb{1} .
$$

The map above is an $\mathcal{A}$-homomorphism, as can be explicitly seen by calculating the commutator between two transformed coordinates:

$$
\begin{aligned}
{\left[\Delta_{L}\left[x^{\mu}\right], \Delta_{L}\left[x^{\nu}\right]\right] } & =\Delta_{L}\left[i\left(v^{\mu} x^{\nu}-v^{\nu} x^{\mu}\right)\right] \\
& =i\left(v^{\mu} \Delta_{L}\left[x^{\nu}\right]-v^{\nu} \Delta_{L}\left[x^{\mu}\right]\right)
\end{aligned}
$$

The above relation is left invariant only if $\Lambda^{\mu}{ }_{\nu}$ and $a^{\mu}$ satisfy the commutation relations (6). 


\section{Scalar fields on $\boldsymbol{\kappa}$-Minkowski and the $\kappa$-Poincaré algebra}

As we remarked above, scalar fields on $\kappa$-Minkowski are simply generic elements of $\mathcal{A}$ (spacetime coordinates are scalar fields too: in the commutative case they are four scalar fields whose values at a spacetime point give the values of the four coordinates of that point in a certain coordinate system). One can define on $\kappa$-Minkowski a notion of Fourier transform, by expanding a generic scalar field $\phi(x)$ in ordered plane waves [16]:

$$
\begin{aligned}
\phi(x) & =\int_{\mathbb{R}^{4}} d^{4} k \tilde{\phi}_{r}(k) e^{i k_{i} x^{i}} e^{i k_{0} x^{0}}=\int_{\mathbb{R}^{4}} d^{4} k \tilde{\phi}_{l}(k) e^{i k_{0} x^{0}} e^{i k_{i} x^{i}} \\
& =\int_{\mathbb{R}^{4}} d^{4} k \tilde{\phi}_{w}(k) e^{i k_{0} x^{0}+i k_{i} x^{i}}
\end{aligned}
$$

Using the commutation relations (9) we can prove the following relationships between waves written with different ordering conventions [57]:

$e^{i k_{0} x^{0}} e^{i k_{i} x^{i}}=e^{i e^{\frac{k_{0}}{\kappa}} k_{i} x^{i}} e^{i k_{0} x^{0}}, \quad e^{i k_{0} x^{0}+i k_{i} x^{i}}=e^{i\left(\frac{k_{0} / k_{0}}{k_{0} / k}\right) k_{i} x^{i}} e^{i k_{0} x^{0}}$,

and therefore the Fourier transform coefficients corresponding to differently ordered waves are related to each other by coordinate transformations of momentum space:

$$
\begin{aligned}
\tilde{\phi}_{r}\left(q_{0}, \mathbf{q}\right) & =e^{-3 q_{0} / \kappa} \tilde{\phi}_{l}\left(q_{0}, e^{-q_{0} / \kappa} \mathbf{q}\right) \\
& =\frac{\left|q_{0} / \kappa\right|^{3}}{\left|e^{q_{0} / \kappa}-1\right|^{3}} \tilde{\phi}_{w}\left(q_{0}, \frac{q_{0} / \kappa}{\left(e^{q_{0} / \kappa}-1\right)} \mathbf{q}\right) .
\end{aligned}
$$

We can find out how the $\kappa$-Poincaré group acts on a scalar field by using the homomorphism property (10) of the left coaction:
$\Delta_{L}[\phi(x)]=\phi\left(\Delta_{L}[x]\right)=\int_{\mathbb{R}^{4}} d^{4} k \tilde{\phi}_{r}(k) e^{i k_{i} \Delta_{L}\left[x^{i}\right]} e^{i k_{0} \Delta_{L}\left[x^{0}\right]}$.

The right-ordered plane waves transform in the following way:

$$
\begin{aligned}
& e^{i k_{i}\left(\Lambda_{\nu}^{i} \otimes x^{\nu}+a^{i} \otimes \mathbb{1}\right)} e^{i k_{0}\left(\Lambda_{\nu}^{0} \otimes x^{\nu}+a^{0} \otimes \mathbb{1}\right)} \\
& \quad=e^{i \lambda_{i}[k, \Lambda] \otimes x^{i}} e^{i \lambda_{0}[k, \Lambda] \otimes x^{0}} e^{i k_{i} a^{i} \otimes \mathbb{1}} e^{i k_{0} a^{0} \otimes \mathbb{1}}
\end{aligned}
$$

where $\lambda_{\mu}[k, \Lambda]$ are four complicated, nonlinear function of $k_{\mu}$ and $\Lambda_{\nu}^{\mu}$, which we calculate explicitly (in the $1+1$ dimensional case) in Appendix A.

At first order in $\omega^{\mu}{ }_{\nu}=\log \Lambda^{\mu}{ }_{\nu}$ and $a^{\mu}$ :

$$
\begin{aligned}
e^{i k_{i} \Delta_{L}\left[x^{i}\right]} e^{i k_{0} \Delta_{L}\left[x^{0}\right]}= & \mathbb{1} \otimes e^{i k_{i} x^{i}} e^{i k_{0} x^{0}}+\omega^{\mu}{ }_{\nu} \otimes M^{\nu}{ }_{\mu} \triangleright e^{i k_{i} x^{i}} e^{i k_{0} x^{0}} \\
& +a^{\mu} \otimes P_{\mu} \triangleright e^{i k_{i} x^{i}} e^{i k_{0} x^{0}}+\ldots
\end{aligned}
$$

The operators $M^{\mu}{ }_{\nu}$ and $P_{\mu}$ are the Lorentz and translation generators of the $\kappa$-Poincaré algebra, which is dual to the quantum group. The symbol $\triangleright$ refers to an action of the $\kappa$-Poincaré algebra on $\mathcal{A}$. From the last formula we deduce that, at first order in the transformation parameters, a scalar field transforms as

$\Delta_{L}[\phi(x)]=\mathbb{1} \otimes \phi+\omega^{\mu}{ }_{\nu} \otimes M^{\nu}{ }_{\mu} \triangleright \phi+a^{\mu} \otimes P_{\mu} \triangleright \phi+\ldots$

The operators $M_{\mu \nu}$ and $P_{\mu}$ close a Hopf algebra $U_{\kappa}[\mathfrak{g}]$, which is dual to $\mathbb{C}_{\kappa}[\mathcal{G}]$. Its commutators are ${ }^{4}$

$$
\begin{aligned}
& {\left[P_{\mu}, P_{\nu}\right]=0, \quad\left[R_{j}, P_{0}\right]=0, \quad\left[R_{j}, P_{k}\right]=i \varepsilon_{j k l} P_{l}, \quad\left[R_{j}, N_{k}\right]=i \varepsilon_{j k l} N_{l}, \quad\left[R_{j}, R_{k}\right]=i \varepsilon_{j k l} R_{l},} \\
& {\left[N_{j}, P_{k}\right]=i P_{j}, \quad\left[N_{j}, P_{k}\right]=\frac{i}{2} \delta_{j k}\left(\kappa\left(1-e^{-2 P_{0} / \kappa}\right)+\frac{1}{\kappa}|\mathbf{P}|^{2}\right)-\frac{i}{\kappa} P_{j} P_{k}, \quad\left[N_{j}, N_{k}\right]=-i \varepsilon_{j k l} R_{l} .}
\end{aligned}
$$

Notice that $\left.\frac{\partial \lambda_{\rho}[P, \Lambda]}{\partial \Lambda^{\mu}{ }_{\nu}}\right|_{\Lambda^{\mu}{ }_{\nu}=\delta^{\mu}{ }_{\nu}}=i\left[M^{\mu}{ }_{\nu}, P_{\rho}\right]$, and therefore the nonlinear commutators $\left[M^{\mu}{ }_{\nu}, P_{\rho}\right]$ encode the action of infinitesimal Lorentz transformations on momentum space. The coproducts, antipodes, and counits of $U_{\kappa}[\mathfrak{g}] \operatorname{are}^{5}$

$$
\begin{gathered}
\Delta\left[P_{j}\right]=P_{j} \otimes \mathbb{1}+e^{-P_{0} / \kappa} \otimes P_{j}, \quad \Delta\left[P_{0}\right]=P_{0} \otimes \mathbb{1}+\mathbb{1} \otimes P_{0}, \\
\Delta\left[R_{j}\right]=R_{j} \otimes \mathbb{1}+\mathbb{1} \otimes R_{j}, \quad \Delta\left[N_{k}\right]=N_{k} \otimes \mathbb{1}+e^{-P_{0} / \kappa} \otimes N_{k}+\frac{i}{\kappa} \varepsilon_{k l m} P_{l} \otimes R_{m}, \\
S\left[P_{0}\right]=-P_{0}, \quad S\left[P_{j}\right]=-e^{P_{0} / \kappa} P_{j}, \quad S\left[R_{j}\right]=-R_{j}, \quad S\left[N_{j}\right]=-e^{P_{0} / \kappa} N_{j}+\frac{i}{\kappa} \varepsilon_{j k l} e^{P_{0} / \kappa} P_{k} R_{l}, \\
\varepsilon\left[P_{0}\right]=0, \quad \varepsilon\left[P_{j}\right]=0, \quad \varepsilon\left[R_{j}\right]=0, \quad \varepsilon\left[N_{j}\right]=0 .
\end{gathered}
$$

${ }^{4} N_{i}=M_{0 i}, R_{i}=\frac{1}{2} \epsilon_{i j k} M_{j k}$

${ }^{5}$ For a nice introduction to $\kappa$-Minkowski and $\kappa$-Poincaré see, for instance, [58,57] for a more formal exposition. 


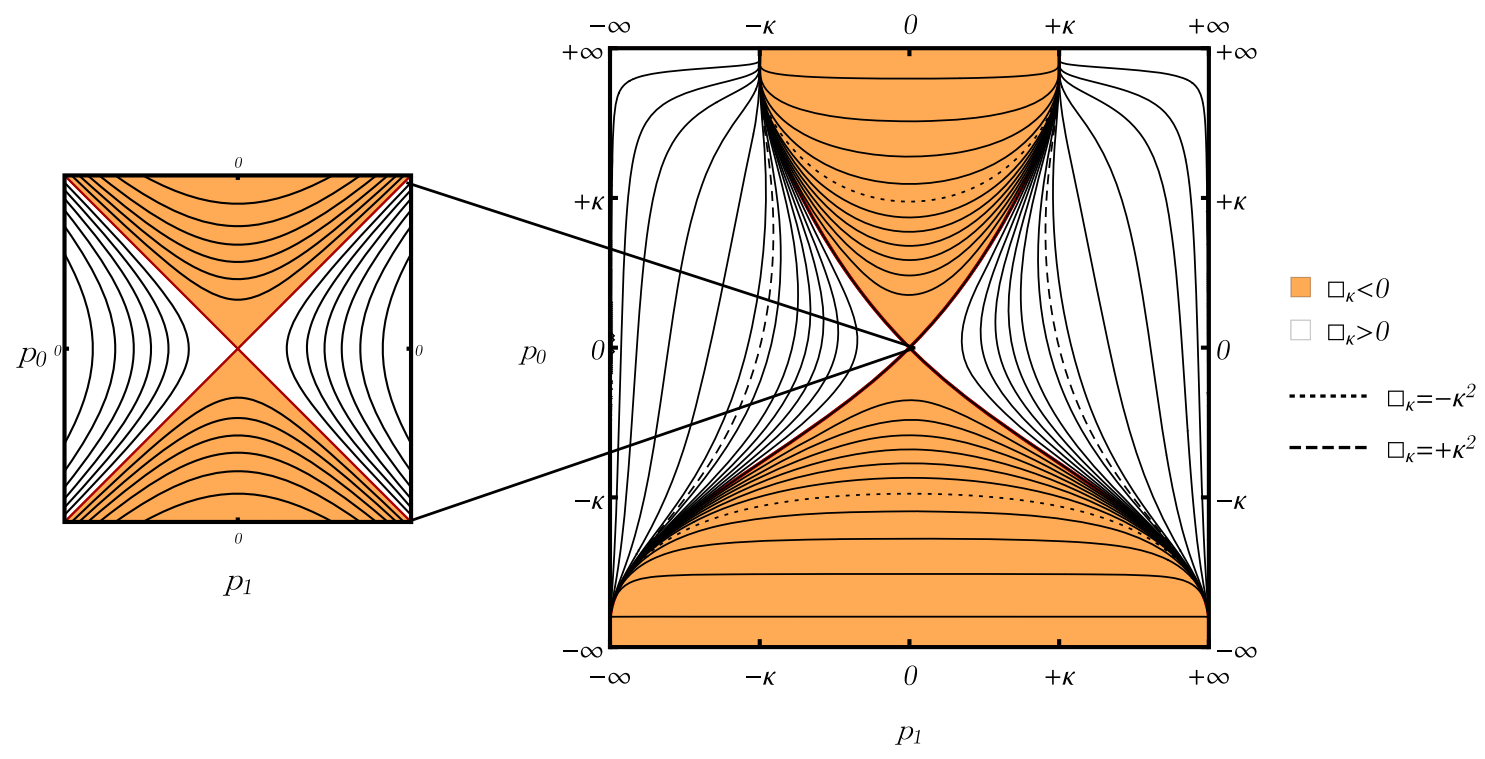

FIG. 1. The $\square_{\kappa}=$ const curves in momentum space in $1+1$ dimensions. Both the vertical $\left(p_{0}\right)$ and the horizontal $\left(p_{1}\right)$ axes have been compactified by taking the arctan of the variable. In a sufficiently small neighborhood of the origin $p_{\mu}=0$, the diagram is indistinguishable from the mass-shell hyperboloids of the momentum space of waves on the commutative Minkowski spacetime. This diagram is a compactified version of the diagram that first appeared in Fig. 2 of [10]. The orange region corresponds to negative $\square_{\kappa}$, which in the $\kappa \rightarrow \infty$ limit coincides with waves with positive squared mass. The white region, instead, tends to tachyonic (negative squared mass) waves in the $\kappa \rightarrow \infty$ limit. Notice how the "future-directed" $\left(p_{0}>0\right)$ and "past-directed" $\left(p_{0}<0\right)$ mass shells are differently shaped. In particular, the future-directed one is spatially bounded $\left|p_{1}\right|<\kappa$ while the past-directed one is not. To give a sense of scale, we plotted differently the on-shell curves with $\square_{\kappa}= \pm \kappa^{2}$.

The coproducts, antipode, and counit are deformations of the relations $\Delta[t]=t \otimes \mathbb{1}+\mathbb{1} \otimes t, S[t]=-t$ and $\varepsilon[t]=0$ which identify the Lie-algebra generator $t$ as a differential operator. In particular $\Delta[t]$ encodes the Leibniz rule for acting on products of functions, through the relation $t \triangleright(f g)=\mu \circ[\Delta[t] \triangleright(f \otimes g)]=(t \triangleright f) g+f(t \triangleright g)$. In the case of $U_{\kappa}[\mathfrak{g}]$ the coproduct above implies that only $P_{0}$ and $R_{i}$ will respect the Leibniz rule when acting on noncommutative products of functions. $P_{j}$ and $N_{j}$ will behave differently, e.g., $P_{j} \triangleright(\phi \psi)=\left(P_{j} \triangleright \phi\right) \psi+$ $\left(e^{-P_{0} / \kappa} \triangleright \phi\right)\left(P_{j} \triangleright \psi\right)$.

The commutators (19) leave invariant the following function of the momentum generators:

$$
\square_{\kappa}=-4 \kappa^{2} \sinh ^{2}\left(\frac{P_{0}}{2 \kappa}\right)+e^{\frac{P_{0}}{\kappa}}|\mathbf{P}|^{2} .
$$

$\square_{\kappa}$ is a high-energy deformation of the quadratic Casimir of the Poincaré algebra. In fact, expanding in powers of $\kappa^{-1}$,

$$
\square_{\kappa}=-P_{0}^{2}+|\mathbf{P}|^{2}+\frac{1}{\kappa} P_{0}|\mathbf{P}|^{2}+\mathcal{O}\left(\kappa^{-2}\right),
$$

which is indistinguishable from $-P_{0}^{2}+|\mathbf{P}|^{2}$ for $P_{0} \ll \kappa$.

\section{The $\kappa$-momentum space}

The time-to-the-right-ordered plane waves $e^{i p_{i} x^{i}} e^{i p_{0} x^{0}}$ are eigenfunctions of the momentum operators $P_{\mu}$ :

$$
P_{\mu} \triangleright e^{i p_{i} x^{i}} e^{i p_{0} x^{0}}=p_{\mu} e^{i p_{i} x^{i}} e^{i p_{0} x^{0}} .
$$

Applying the $\kappa$-deformed Casimir operator (23) to a plane wave,

$\square_{\kappa} \triangleright e^{i p_{i} x^{i}} e^{i p_{0} x^{0}}=\left(-4 \kappa^{2} \sinh ^{2}\left(\frac{p_{0}}{2 \kappa}\right)+e^{\frac{p_{0}}{\kappa}}|\mathbf{k}|^{2}\right) e^{i p_{i} x^{i}} e^{i p_{0} x^{0}}$,

we can introduce a notion of an "on-shell" wave, which satisfies a $\kappa$-deformed version of the Klein-Gordon equation:

$$
-4 \kappa^{2} \sinh ^{2}\left(\frac{p_{0}}{2 \kappa}\right)+e^{\frac{p_{0}}{\kappa}}|\mathbf{k}|^{2}=\text { const. }
$$

Now consider the on-shell curves, in the space of momentum eigenvalues $p_{\mu} \in \mathbb{R}^{4}$, associated with constant values of $\square_{\kappa}$. In Fig. 1 we see that these curves are a high-energy deformation of the on-shell curves of Minkowski space.

We can study the action of Lorentz transformations on momentum space. In $1+1 \mathrm{D}$, boost transformations of Eq. (16) can be written as (for the proof, see Appendix A) 

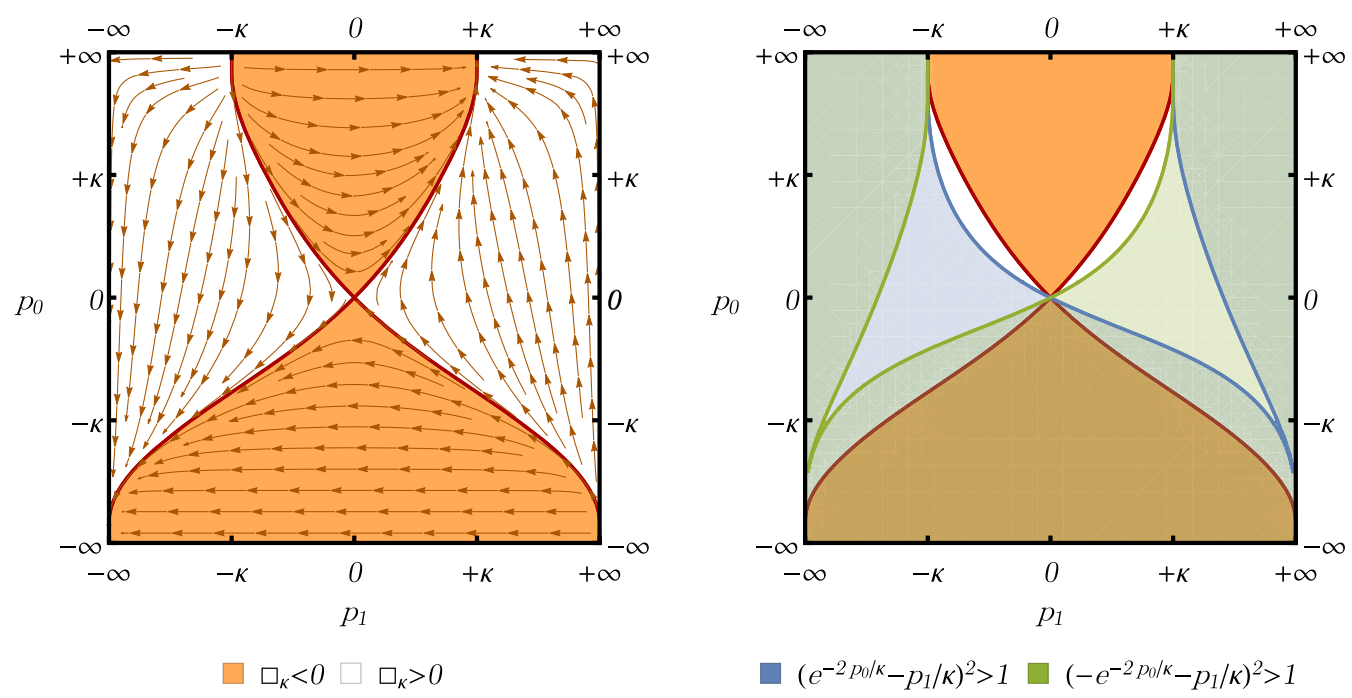

FIG. 2. Left: Lorentz vector field on momentum space. Right: regions where a critical rapidity exists. In the superposition between the blue and the green regions, there exist two values of the rapidity that make the boosted momenta diverge.

$$
\begin{aligned}
& \lambda_{0}[\xi, p]=p_{0}+\kappa \log \left[\left(\cosh \frac{\xi}{2}+\frac{p_{1}}{\kappa} \sinh \frac{\xi}{2}\right)^{2}-e^{-2 p_{0} / \kappa} \sinh ^{2} \frac{\xi}{2}\right] \\
& \lambda_{1}[\xi, p]=\kappa \frac{\left(\cosh \frac{\xi}{2}+\frac{p_{1}}{\kappa} \sinh \frac{\xi}{2}\right)\left(\sinh \frac{\xi}{2}+\frac{p_{1}}{\kappa} \cosh \frac{\xi}{2}\right)-e^{-2 p_{0} / \kappa} \cosh \frac{\xi}{2} \sinh \frac{\xi}{2}}{\left(\cosh \frac{\xi}{2}+\frac{p_{1}}{\kappa} \sinh \frac{\xi}{2}\right)^{2}-e^{-2 p_{0} / \kappa} \sinh ^{2} \frac{\xi}{2}}
\end{aligned}
$$

We plot the flux of the above transformation (i.e., the vector field $\frac{\partial \lambda_{\mu}}{\partial \xi} \frac{\partial}{\partial p_{\mu}}$ ) in Fig. 2 (on the left).

Notice that both components of Eq. (28) have a divergence at

$$
\left(\cosh \frac{\xi}{2}+\frac{p_{1}}{\kappa} \sinh \frac{\xi}{2}\right)^{2}-e^{-2 p_{0} / \kappa} \sinh ^{2} \frac{\xi}{2}=0,
$$

which means

$$
\operatorname{coth} \frac{\xi}{2}= \pm e^{2 p_{0} / \kappa}-\frac{p_{1}}{\kappa} .
$$

Now, the image of coth is $(-\infty,-1) \cup(1,+\infty)$, and therefore the above equation admits a solution in $\xi$ only wherever $\left| \pm e^{2 p_{0} / \kappa}-\frac{p_{1}}{\kappa}\right|>1$. This completely excludes the positive-frequency mass shell, and part of the $\square_{\kappa}>0$ white region of Fig. 1. We plot the two regions $\left|e^{2 p_{0} / \kappa}-\frac{p_{1}}{\kappa}\right|>1$ and $\left|-e^{2 p_{0} / \kappa}-\frac{p_{1}}{\kappa}\right|>1$ in Fig. 2 (on the right). The existence of this "critical rapidity" was first noticed by Majid [10]. As can be seen in Fig. 2, the positive-frequency $\square_{\kappa}<0$ shell does not have a critical rapidity: in there, any finite value of $\xi$ corresponds to a finite boosted momentum. All the other regions of momentum space, however, have this issue, and the rapidities can only take either a finite interval or an interval bounded from above or below, because with a finite value of the rapidity one is boosted to the boundary of momentum space. This is in principle a serious issue which might spoil any theory based on this symmetry group of the equivalence between inertial observers. Tackling this issue will be an important part of our results.

As we remarked in (13), changing the ordering coincides with a nonlinear redefinition of the eigenvalue $k_{\mu}$, e.g., $k_{i} \rightarrow e^{\frac{k_{0}}{\kappa}} k_{i}, k_{0} \rightarrow k_{0}$ to go from the right-ordered to the leftordered plane waves. This, in turn, means that the leftordered plane waves are eigenfunctions of the operators $P_{i}^{l}=e^{\frac{P_{0}}{\kappa}} P_{i}$, which are just a different basis for the $U_{\kappa}[\mathfrak{g}]$ algebra. In Fig. 3 we plot the on-shell curves in the Weylordered and the time-to-the-left-ordered coordinates. We can see that most qualitative features of these diagrams depend on the ordering choice, and therefore on the Hopf algebra basis.

\section{E. The geometry of momentum space}

More than one author could not resist the temptation to interpret expressions like (24) literally, as relations between the physical (observable) energy and momentum carried by a wave in a noncommutative spacetime. Then one would be led to calculate things like the group velocity of the wave as the slope of the on-shell curves, $\partial p_{0} / \partial p_{1}$, and deduce, e.g., that the positive-frequency waves of Fig. 1 have an infinite group velocity as the spatial momentum approaches 

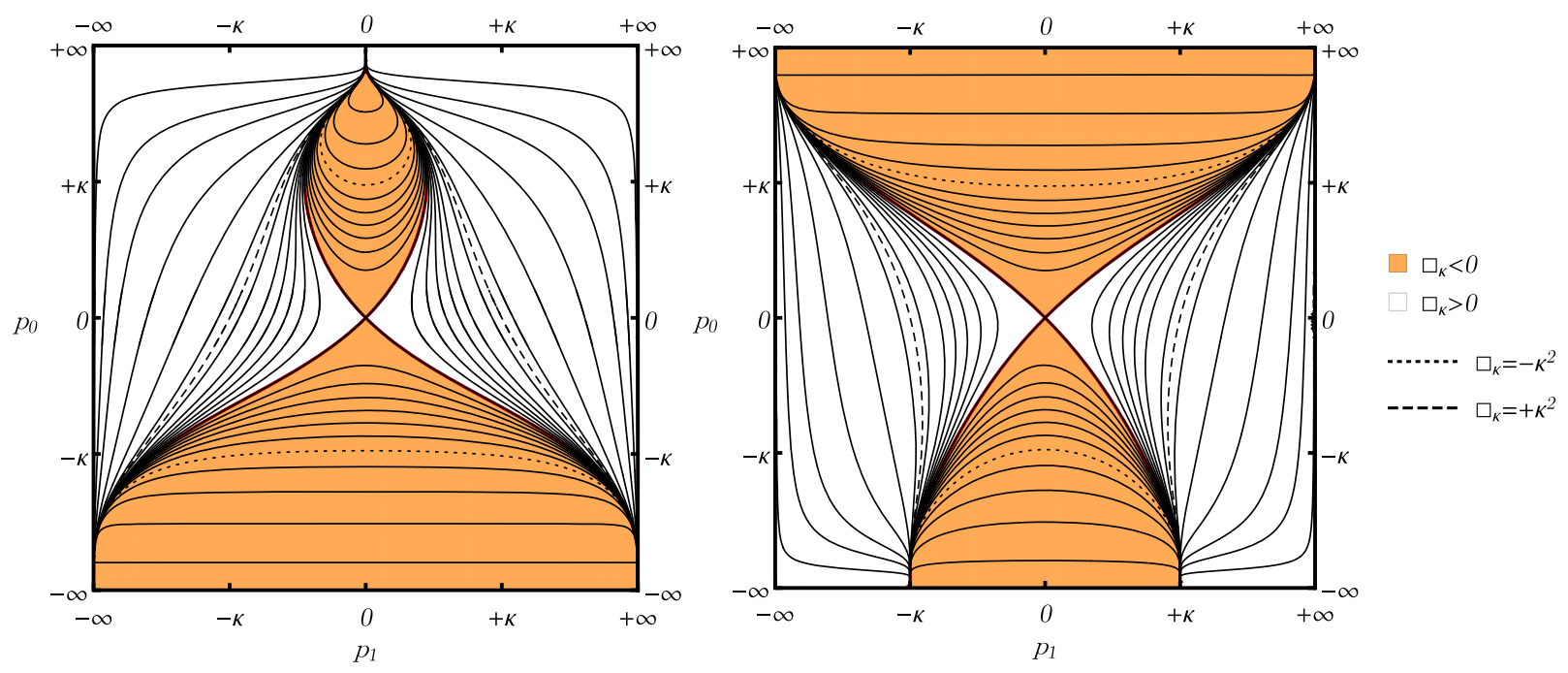

FIG. 3. The $\square_{\kappa}=$ const curves in Weyl-ordered coordinates (left), and time-to-the-left-ordered coordinates (right).

$\left|p_{1}\right| \rightarrow \kappa$. The group velocity of negative-frequency waves instead would become infinite only as $\left|p_{1}\right| \rightarrow \infty$. However, if we adopted the convention of ordering our waves with the time to the left, positive- and negative-frequency waves would swap their behaviors, as suggested by the diagram on the right-hand side of Fig. 3. Even more strange would be the behavior of the Weyl-ordered waves: the left-hand side of Fig. 3 suggests that positive-frequency waves have a multivalued dispersion relation, that associates two different energies to each spatial momentum (and the group velocity would diverge at a value of $\left|p_{1}\right|$ that is even smaller than $\kappa$ ).

It would of course be preferable to avoid the conclusion that physics depends on the convention we adopt to order our operators. Considering different ordering choices, however, suggests the way out of this problem: changing ordering corresponds to making a general coordinate transformation on our momentum space. Then we should be looking for statements that are coordinate independent: those that regard the pseudo-Riemannian geometry of momentum space.

Kowalski-Glikman was the first to observe that the momentum space of $\kappa$-Minkowski has the geometry of a de Sitter manifold [31]. Here we would like to repeat the argument presented in [30], which starts with the observation that there exists a change of basis:

$$
\eta_{0}=\kappa \sinh \frac{P_{0}}{\kappa}+\frac{1}{2 \kappa} e^{\frac{P_{0}}{\kappa}}|\mathbf{P}|^{2}, \quad \eta_{i}=e^{\frac{P_{0}}{\kappa}} P_{i},
$$

such that $\eta_{\mu}$ closes a Poincaré algebra with the Lorentz generators $M_{\mu \nu}$ :

$$
\left[M_{\mu \nu}, \eta_{\rho}\right]=i \eta_{\mu \rho} \eta_{\nu}-i \eta_{\nu \rho} \eta_{\mu}
$$

as can be explicitly deduced from the definition of $\eta_{\mu}$ and the commutation relations (19). The generators $\eta_{\mu}$ transform classically under Lorentz transformations. However they do not close a Hopf algebra: their coproducts are

$\Delta\left[\eta_{0}\right]=\eta_{0} \otimes e^{P_{0} / \kappa}+e^{-P_{0} / \kappa} \otimes \eta_{0}+\frac{1}{\kappa} e^{-P_{0} / \kappa} \eta_{i} \otimes \eta_{i}$,

$\Delta\left[\eta_{i}\right]=\eta_{i} \otimes e^{P_{0} / \kappa}+\mathbb{1} \otimes \eta_{i}$,

$S\left[\eta_{0}\right]=-\eta_{0}+e^{-P_{0} / \kappa}|\eta|^{2}, \quad S\left[\eta_{i}\right]=-e^{-P_{0} / \kappa} \eta_{i}$,

but we cannot express $e^{P_{0} / \kappa}$ uniquely in terms of $\eta_{0}, \eta_{1}$. The reason is that Eq. (31) as a transformation $\left(P_{0}, P_{i}\right) \rightarrow$ $\left(\eta_{0}, \eta_{i}\right)$ is not injective, because it admits two inversions:

$$
\begin{aligned}
e^{\frac{P_{0}}{\kappa}} & =\eta_{0} \pm \sqrt{\eta_{0}^{2}-|\boldsymbol{\eta}|^{2}-\kappa^{2}}, \\
P_{i} & =\eta_{i} \frac{\kappa\left(\eta_{0} \mp \sqrt{\eta_{0}^{2}-|\boldsymbol{\eta}|^{2}-\kappa^{2}}\right)}{|\boldsymbol{\eta}|^{2}-\kappa^{2}},
\end{aligned}
$$

so the coalgebra does not close. If we now introduce a new algebra element,

$$
\eta_{4}=\kappa \cosh \frac{P_{0}}{\kappa}-\frac{1}{2 \kappa} e^{\frac{P_{0}}{\kappa}}|\mathbf{P}|^{2}
$$

the coordinate transformation $\left(P_{0}, P_{i}\right) \rightarrow\left(\eta_{0}, \eta_{i}, \eta_{4}\right)$ becomes injective (although it is not surjective, because the target space is 5-dimensional)

$$
e^{\frac{P_{0}}{\kappa}}=\frac{\eta_{0}+\eta_{4}}{\kappa}, \quad P_{i}=\eta_{i} \frac{\kappa\left(\eta_{0}-\eta_{4}\right)}{|\boldsymbol{\eta}|^{2}-\kappa^{2}},
$$

and then the coproducts close on the $\left(\eta_{0}, \eta_{i}, \eta_{4}\right)$ basis: 

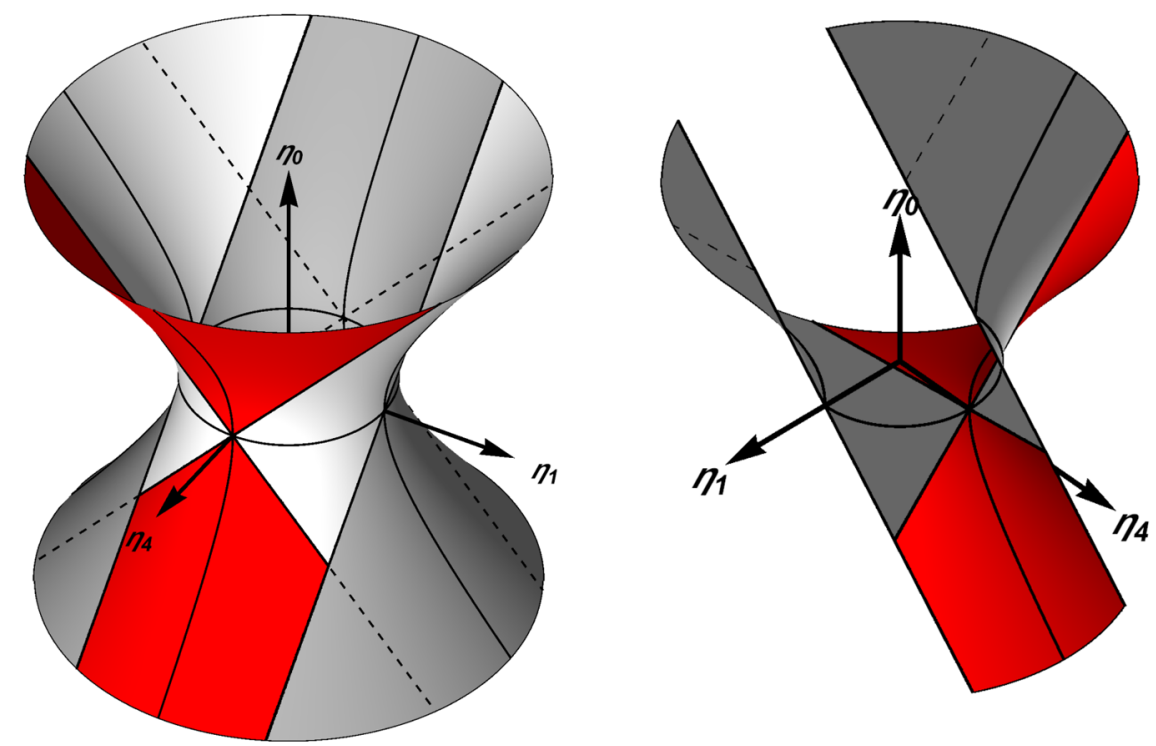

FIG. 4. Left: $\kappa$-Minkowski's de Sitter momentum space in embedding coordinates $\eta_{a}$. The dark gray area is not covered by the bicross-product coordinates. The red and white areas correspond, respectively, to $\square_{\kappa}<0$ and $\square_{\kappa}>0$. Right: part of de Sitter space that is covered by bi-cross-product coordinates, from a different perspective.

$\Delta\left[\eta_{0}\right]=\eta_{0} \otimes\left(\eta_{0}+\eta_{4}\right)+\frac{\kappa}{\eta_{0}+\eta_{4}} \otimes \eta_{0}+\frac{\eta_{i}}{\eta_{0}+\eta_{4}} \otimes \eta_{i}$,

$\Delta\left[\eta_{i}\right]=\frac{1}{\kappa} \eta_{i} \otimes\left(\eta_{0}+\eta_{4}\right)+\mathbb{1} \otimes \eta_{i}$,

$\Delta\left[\eta_{4}\right]=\eta_{4} \otimes\left(\eta_{0}+\eta_{4}\right)-\frac{\kappa}{\eta_{0}+\eta_{4}} \otimes \eta_{0}-\frac{\eta_{i}}{\eta_{0}+\eta_{4}} \otimes \eta_{i}$,

as well as the antipodes:

$S\left[\eta_{0}\right]=-\eta_{0}+\frac{|\eta|^{2}}{\eta_{0}+\eta_{4}}, \quad S\left[\eta_{i}\right]=-\frac{\kappa \eta_{i}}{\eta_{0}+\eta_{4}}, \quad S\left[\eta_{4}\right]=\eta_{4}$.

Notice that the definitions of $\eta_{a}=\eta_{a}\left(P_{0}, \mathbf{P}\right), a=$ $0, \ldots, 4$ are such that the five $\eta_{a}$ are not independent: they satisfy an algebraic relation

$$
\eta_{0}^{2}-|\boldsymbol{\eta}|^{2}-\eta_{4}^{2}=-\kappa^{2}
$$

which is the equation for the 4D de Sitter hyperboloid in 5-dimensional embedding coordinates. Together with the counits,

$$
\varepsilon\left[\eta_{0}\right]=0, \quad \varepsilon\left[\eta_{i}\right]=0, \quad \varepsilon\left[\eta_{4}\right]=\kappa,
$$

the coproducts (38) and antipodes (39) satisfy the Hopf algebra axiom (5) only on shell, that is, after imposing Eq. (40).

We conclude that $\eta_{a}=\eta_{a}\left(P_{0}, \mathbf{P}\right): \mathbb{R}^{4} \rightarrow d S_{4}$ are a coordinatization of de Sitter space. Differently ordered bases just correspond to different ways of coordinatizing de Sitter space with $\mathbb{R}^{4}$. In other words, different bases are related by diffeomorphisms. The metric induced on $d S$ by the flat ambient metric,

$$
\begin{aligned}
\mathrm{d} s^{2} & =-\mathrm{d} \eta_{0}^{2}+\mathrm{d} \eta_{1}^{2}+\mathrm{d} \eta_{2}^{2}+\mathrm{d} \eta_{3}^{2}+\mathrm{d} \eta_{4}^{2} \\
& =-\mathrm{d} P_{0}^{2}+e^{2 P_{0} / \kappa}\left(\mathrm{d} P_{1}^{2}+\mathrm{d} P_{2}^{2}+\mathrm{d} P_{3}^{2}\right),
\end{aligned}
$$

is, in right-ordered bicrossproduct coordinates, the de Sitter metric in comoving coordinates:

$$
g_{\mu \nu}=\operatorname{diag}\left(-1, e^{2 P_{0} / \kappa}, e^{2 P_{0} / \kappa}, e^{2 P_{0} / \kappa}\right),
$$

so we may conclude that right-ordered coordinates are comoving coordinates on momentum space.

Notice now that the coordinates $\eta_{a}=\eta_{a}\left(P_{0}, \mathbf{P}\right)$ only cover half of de Sitter space. In fact from Eq. (37) we see that, for $P_{0}$ real, $\eta_{0}+\eta_{4}=\kappa e^{\frac{P_{0}}{\kappa}}>0$. The region $\eta_{0}+\eta_{4}$ cuts the de Sitter hyperboloid in the way shown in Fig. 4.

Equation (32) implies that the Lorentz transformations act in an undeformed way on the $\eta_{\mu}$ coordinates. The $\eta_{4}$ coordinate, on the other hand, is Lorentz invariant; in fact, it is a function of the Casimir (23):

$$
\eta_{4}=\kappa-\frac{\square_{\kappa}}{2 \kappa} .
$$

Then the Lorentz group acts on the de Sitter momentum space as the group of isometries that stabilize the "origin" point $^{6} \eta_{4}=\kappa, \eta_{\mu}=0$ (see Fig. 5). It is obvious that these

\footnotetext{
${ }^{6}$ Notice how the coordinates of the origin point are given by the counits of $\eta_{a}: \varepsilon\left(\eta_{4}\right)=\kappa, \varepsilon\left(\eta_{\mu}\right)=0$.
} 

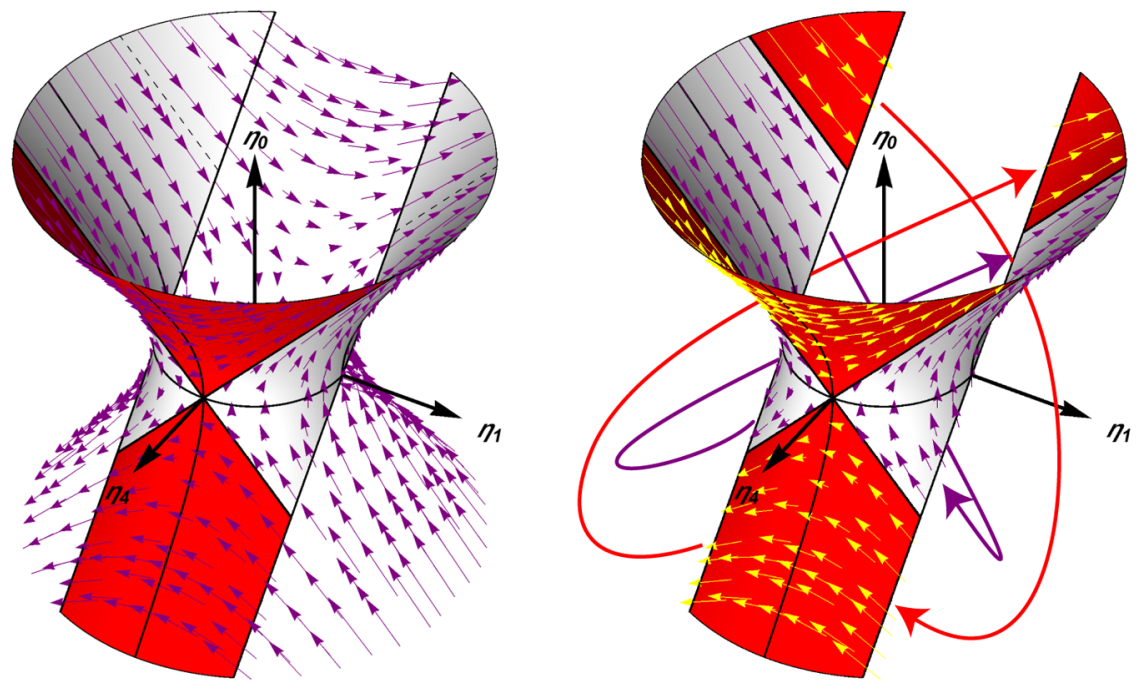

FIG. 5. Left: Lorentz flow on de Sitter momentum space. It is apparent that a finite boost can bring a point outside of the region covered by the bi-cross-product coordinates. Right: Elliptic boundary conditions on the patch of $d S$ momentum space covered by the bi-cross-product coordinates, and how to continue the Lorentz vector flow through the boundaries. Notice how the region that is connected by Lorentz transformations to the past light cone of the origin is now in red.

transformations do not leave the region covered by the bi-cross-product coordinates, $\eta_{0}+\eta_{4}>0$, invariant. Moreover it takes a finite rapidity to bring a point in the region $\eta_{0}+\eta_{4}>0$ outside of it, unless this point is in the future light cone of the origin - the only region which is closed under Lorentz transformations.

The region $\eta_{0}+\eta_{4}>0$ is sent to its complement by reflections $\eta_{a} \rightarrow-\eta_{a}$. Consequently, if we quotient de Sitter space by reflections, we obtain a manifold that is covered uniquely by the bi-cross-product coordinates. This

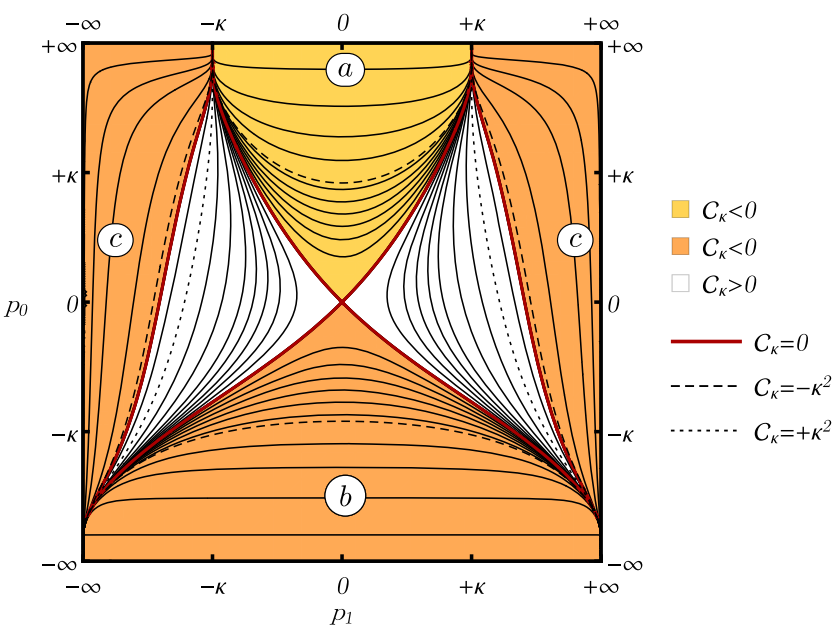

FIG. 6. Momentum space in bi-cross=product coordinates. The negative- $\mathcal{C}_{\kappa}$ region (the one with real mass) has been divided into its two connected components under Lorentz transformations: the positive-frequency one $a$ (in yellow) and the negative-frequency one, $b$ and $c$ (in orange). Moreover some special curves have been highlighted: the zero-mass one (in red), one with minimal value of $\mathcal{C}_{\kappa}=-\kappa^{2}$, and the one with $\mathcal{C}_{\kappa}=+\kappa^{2}$. manifold is a known and well-studied example of non-timeorientable solution of Einstein's equations: it is called "elliptic de Sitter spacetime" $[24,59], d S / \mathbb{Z}_{2}$. This manifold is motivated by the fact that the whole $d S$ hyperboloid is "too much" as a cosmological model. In fact, unlike what happens in Minkowski spacetime, an observer located on a timelike curve cannot be in causal contact with more than "half" of de Sitter space. The causal domain of a timelike curve is precisely a region of the same form as $\eta_{0}+\eta_{4}>0$. (All of these causal domains can be obtained by rotating $\eta_{0}+\eta_{4}>0$ around the $\eta_{0}$ axis. They are parametrized only by the endpoint of their defining worldline on the boundary of $d S$ space. $^{7}$ )

Both $d S$ and $d S / \mathbb{Z}_{2}$ locally solve Einstein's equations with a positive cosmological constant, under the assumption of spacetime homogeneity. What distinguishes them is the assumed topology. Similarly, in realizing that the $\kappa$-Poincare momentum space has a $d S$ geometry, we used the local structure of its symmetries (the $\kappa$-Poincaré algebra) while tacitly making an assumption regarding its global topology, which in truth we are free to choose. The global behavior of the Lorentz flow on momentum space reveals that the standard $d S$ topology $\mathbb{R} \times S_{3}$ is not adequate, as it leads to a singular Lorentz flow. We assume

\footnotetext{
${ }^{7}$ One can convince herself of this by observing that the past light cone of a point on a timelike worldline is included in the past light cone of any future point on the worldline. Therefore, to find the causally connected region to the whole worldline, it is sufficient to consider the past light cone of the point on the worldline at the infinite boundary of $d S$ spacetime. This is given, in the ambient space, by a half-space delimited by a $45^{\circ}$ plane which intersects the center of the $d S$ hyperboloid. One such plane is $\eta_{0}+\eta_{4}=0$.
} 
the $d S / \mathbb{Z}_{2}$ topology solves this issue, because now the Lorentz flow lines close.

Now, under the elliptic identification the Casimir $\square_{\kappa}$ is not continuous: it is negative in the orange region of Fig. 1, but it changes sign when a Lorentz transformation brings us from the "past light cone" region through its boundary into the negative- $\eta_{4}$ region. The way the elliptic identification is defined, a Lorentz transformation bringing us through the boundary of the bi-cross-product patch will preserve the absolute value of $\eta_{4}$ but flip its sign. $\square_{\kappa}$ is therefore not a good generalization of the D'Alembert operator, as it fails to be Lorentz -invariant at the global level. In particular, upon crossing the boundary of the bi-cross-product patch, $\square_{\kappa} \rightarrow 4 \kappa^{2}-\square_{\kappa}$. Then it is obvious that any function of the quantity

$$
\mathcal{C}_{\kappa}=\left(1-\frac{\square_{\kappa}}{4 \kappa^{2}}\right) \square_{\kappa}
$$

will be genuinely Lorentz invariant all along any Lorentz orbit. The above function on momentum space will be our choice for the D'Alembert operator. It is negative definite in the on-shell regions and unbounded. Moreover it reduces to $\square$ in the $\kappa \rightarrow \infty$ limit. This operator was already proposed as the generalization of the D'Alembert operator in [13] and used also in other works, like [24]. However, the authors of [13] had a different reason to introduce $\mathcal{C}_{\kappa}$ : one obtains the same operator by defining the D'Alembert operator as $\eta_{\mu} \eta^{\mu}$, where the four $\eta_{\mu}$ are those defined in (31).

\section{FREE QUANTUM $\boldsymbol{\kappa}$-KLEIN-GORDON FIELD}

Now that we know that momentum space is a pseudoRiemannian manifold, we can observe that the noncommutative functions written in Fourier transform with respect to a basis of ordered plane waves, as in (12), have Fourier coefficients $\tilde{\phi}_{r}$ or $\tilde{\phi}_{w}$ that transform, under a change of ordering (that is, a diffeomorphism of momentum space), as scalar densities. Equation (14) expresses this fact: the factors $e^{-3 q_{0} / \kappa}$ and $\frac{\left|q_{0} / \kappa\right|^{3}}{\left|e^{q_{0} / \kappa}-1\right|^{3}}$ are nothing else than the
Jacobians $\operatorname{det}\left(\partial q_{\mu} / \partial q_{\nu}^{\prime}\right)$ of the coordinate transformations $\left(q_{0}^{\prime}, \mathbf{q}^{\prime}\right)=\left(q_{0}, e^{-q_{0} / \kappa} \mathbf{q}\right)$ and, respectively, $\left(q_{0}^{\prime}, \mathbf{q}^{\prime}\right)=$ $\left(q_{0}, q_{0} \mathbf{q} /\left(\kappa e^{q_{0} / \kappa}-\kappa\right)\right)$. Then we can write the Fourier coefficients, e.g., in the right-ordered coordinates, as a scalar field on momentum space times a volume density

$$
\sqrt{-g(k)}=\sqrt{-\operatorname{det} g_{\mu \nu}(k)}=e^{3 \frac{k_{0}}{\kappa}},
$$

and a scalar field $\phi(x)$ can be written, in right-ordered momentum space coordinates:

$$
\phi(x)=\int d^{4} k \sqrt{-g(k)} \phi_{r}(k) e^{i k_{i} x^{i}} e^{i k_{0} x^{0}},
$$

where $g_{\mu \nu}$ is the $d S$ metric in comoving coordinates introduced in Eq. (43).

Our noncommutative generalization of the complex Klein-Gordon equation will be

$$
\mathcal{C}_{\kappa} \triangleright \phi=-m^{2} \phi, \quad \mathcal{C}_{\kappa} \triangleright \phi^{\dagger}=-m^{2} \phi^{\dagger},
$$

A solution to the above equations of motion can be written as

$$
\begin{aligned}
\phi(x)= & \frac{1}{2 m} \int \mathrm{d}^{4} p \sqrt{-g(p)} \sqrt{-g^{\mu \nu}(p) \frac{\partial \mathcal{C}_{\kappa}}{\partial p^{\mu}} \frac{\partial \mathcal{C}_{\kappa}}{\partial p^{\nu}}} \\
& \times \delta\left(\mathcal{C}_{\kappa}(p)+m^{2}\right) \phi_{r}(p): e^{i p_{\mu} x^{\mu}}:
\end{aligned}
$$

where the expression $\sqrt{-g^{\mu \nu}(p) \frac{\partial \mathcal{C}_{\kappa}}{\partial p^{\mu}} \frac{\partial \mathcal{C}_{\kappa}}{\partial p^{\nu}}}$ makes the integral invariant under reparametrizations of the on-shell curve, and the numerical factor $\frac{1}{2 m}$ ensures that the expression has the right commutative limit. The previous expression is invariant under diffeomorphisms in momentum space.

We can solve the on-shell condition $\mathcal{C}_{\kappa}(p)=-m^{2}$ with respect to the $p_{0}$ coordinate, and rewrite the delta function term as

$$
\begin{aligned}
\frac{1}{2 m} \sqrt{-g^{\mu \nu}(p) \frac{\partial \mathcal{C}_{\kappa}}{\partial p^{\mu}} \frac{\partial \mathcal{C}_{\kappa}}{\partial p^{\nu}}} \delta\left(\mathcal{C}_{\kappa}+m^{2}\right) & =\frac{\sqrt{\kappa^{2}+m^{2}}}{\kappa} \delta\left(\mathcal{C}_{\kappa}+m^{2}\right) \\
& =\frac{1}{2 \sqrt{m^{2}+|\mathbf{p}|^{2}}}\left[\delta\left(p_{0}-\omega^{+}(|\mathbf{p}|)\right)+\delta\left(p_{0}-\omega^{-}(|\mathbf{p}|)\right)\right],
\end{aligned}
$$

with

$$
\omega^{ \pm}(|\mathbf{p}|)=-\frac{1}{2} \kappa \log \left(1+\frac{2 m^{2}+|\mathbf{p}|^{2} \mp 2 \sqrt{\left(\kappa^{2}+m^{2}\right)\left(m^{2}+|\mathbf{p}|^{2}\right)}}{\kappa^{2}}\right),
$$

which tends to the usual $\pm \sqrt{\mathbf{p}^{2}+m^{2}}$ in the $\kappa \rightarrow \infty$ limit. Notice that the $\omega^{+}(|\mathbf{p}|)$ solutions split into two, one set defined only in the region $|\mathbf{p}|<\kappa$ and the other in $|\mathbf{p}|>\kappa$. This is because at $|\mathbf{p}|=\kappa, \omega^{-}(|\mathbf{p}|)$ has a (coordinate) singularity: 


$$
\lim _{|\mathbf{p}| \rightarrow \kappa} \omega^{+}(|\mathbf{p}|)=+\infty
$$

The two parts of $\omega^{+}(|\mathbf{p}|)$ belong to the regions $a$ (when $|\mathbf{p}|<\kappa$ ) and $c$ (when $|\mathbf{p}|>\kappa$ ) of momentum space. For this reason, when integrating Eq. (49) with respect to $p_{0}$ we write

$$
\begin{aligned}
\phi(x)= & \int_{|\mathbf{p}|<\kappa} \frac{\mathrm{d}^{3} p e^{\frac{3 \omega^{+}(\mid \mathbf{p})}{\kappa}}}{2 \sqrt{m^{2}+|\mathbf{p}|^{2}}} \phi_{a}(\mathbf{p}) e^{i \mathbf{p} \cdot \mathbf{x}} e^{i \omega^{+}(|\mathbf{p}|) x^{0}}+\int_{\mathbb{R}^{3}} \frac{\mathrm{d}^{3} \mathbf{p} e^{\frac{3 \omega^{-}(\mid \mathbf{p})}{\kappa}}}{2 \sqrt{m^{2}+|\mathbf{p}|^{2}}} \phi_{b}(\mathbf{p}) e^{i \mathbf{p} \cdot \mathbf{x}} e^{i \omega^{-}(|\mathbf{p}|) x^{0}} \\
& +\int_{|\mathbf{p}|>\kappa} \frac{\mathrm{d}^{3} \mathbf{p} e^{\frac{3 \omega^{+}(\mathbf{p} \mid)}{\kappa}}}{2 \sqrt{m^{2}+|\mathbf{p}|^{2}}} \phi_{c}(\mathbf{p}) e^{i \mathbf{p} \cdot \mathbf{x}} e^{i \omega^{+}(|\mathbf{p}|) x^{0}}
\end{aligned}
$$

The coefficients $\phi_{a}(\mathbf{p})$ are closed under Lorentz flow, while the coefficients $\phi_{b}(\mathbf{p})$ and $\phi_{c}(\mathbf{p})$ flow into each other.

The delta function (49) should not be recasted in terms of an integral over a contour on the complex plane of $p_{0}$ (as was done in early works like $[11,13])$. In fact, in doing so, one necessarily breaks the invariance under diffeomorphisms of momentum space, which has been one of the guiding principles of our current analysis. A manifestation of this problem is the fact that the function $\left(\mathcal{C}_{\kappa}(p)+m^{2}\right)$ has infinitely many zeroes in the complex plane of $p_{0}$ :

$$
p_{0}=\omega^{ \pm}(|\mathbf{p}|)+i \pi \kappa n, \quad n \in \mathbb{Z} .
$$

This is due to the fact that $\mathcal{C}_{\kappa}(p)$ depends on $p_{0}$ only through the function $e^{\frac{p_{0}}{2 \kappa}}$ and therefore it is periodic in the imaginary direction, with period $\pi$. But a simple change of variable, e.g., $q=e^{\frac{p_{0}}{2 \kappa}}$, removes all of the nonreal zeroes from the complex plane of $q$. It is clear then that complexifying one coordinate is a nondiffeomorphism-invariant operation.
In $[11,13]$ the complex zeroes of $\mathcal{C}_{\kappa}(p)$ were interpreted as poles of the Green function, and a summation over this infinite tower of poles was proposed. We believe that such a proposal violates one of the basic symmetries of the theory (namely, the independence on the coordinate system in momentum space), and therefore it is not tenable. Fortunately one is not obligated to use residues and contour integrals for what we are concerned with in this paper, and we will be able to use more geometric alternatives, which are explicitly invariant under general changes of coordinates in momentum space.

A classical scalar field is an element of the algebra $\mathcal{A}$. In the noninteracting case, scalar fields are quantized by simply replacing the coefficients $a(\mathbf{p})$ and $b(\mathbf{p})$ with operators on a Hilbert space $\mathcal{H}$ (Fock space). The natural generalization of this to the noncommutative case is to take quantum fields to be elements of $\mathcal{A} \otimes \mathcal{H}$, and replace $\phi_{a}(\mathbf{p}), \phi_{b}(\mathbf{p})$ and $\phi_{c}(\mathbf{p})$ with operators on $\mathcal{H}$. Then this will be our definition of a noncommutative quantum scalar field:

$$
\begin{aligned}
\hat{\phi}(x)= & \int_{|\mathbf{p}|<\kappa} \frac{\mathrm{d}^{3} p e^{\frac{\left.3 \omega^{+}(\mid \mathbf{p})\right)}{\kappa}}}{2 \sqrt{m^{2}+|\mathbf{p}|^{2}}} \hat{a}(\mathbf{p}) e^{i \mathbf{p} \cdot \mathbf{x}} e^{i \omega^{+}(|\mathbf{p}|) x^{0}}+\int_{\mathbb{R}^{3}} \frac{\mathrm{d}^{3} p e^{\frac{3 \omega^{-}(\mathbf{p} \mid)}{\kappa}}}{2 \sqrt{m^{2}+|\mathbf{p}|^{2}}} \hat{b}^{\dagger}(\mathbf{p}) e^{i \mathbf{p} \cdot \mathbf{x}} e^{i \omega^{-}(|\mathbf{p}|) x^{0}} \\
& +\int_{|\mathbf{p}|>\kappa} \frac{\mathrm{d}^{3} p e^{\frac{3 \omega^{+}(\mathbf{p} \mid)}{\kappa}}}{2 \sqrt{m^{2}+|\mathbf{p}|^{2}}} \hat{c}^{\dagger}(\mathbf{p}) e^{i \mathbf{p} \cdot \mathbf{x}} e^{i \omega^{+}(|\mathbf{p}|) x^{0}} .
\end{aligned}
$$

\section{A. Hermitian conjugacy}

$\mathcal{C}_{\kappa} \triangleright \phi=-m^{2} \phi$ implies $\mathcal{C}_{\kappa} \triangleright \phi^{\dagger}=-m^{2} \phi^{\dagger}$, because the Hermitian conjugate acts on (49) by replacing : $e^{i \mathbf{p}_{\mu} x^{\mu}}:$ with $: e^{i S\left(\mathbf{p}_{\mu}\right) x^{\mu}}:$, and the Klein-Gordon operator is invariant under antipode: $\mathcal{C}_{\kappa}(S(\mathbf{p}))=\mathcal{C}_{\kappa}(\mathbf{p})$. To find how Hermitian conjugacy acts on creation and annihilation operators, we first write

$$
\begin{aligned}
\hat{\phi}^{\dagger}(x)= & \int_{|\mathbf{p}|<\kappa} \frac{\mathrm{d}^{3} p e^{\frac{\left.3 \omega^{+}(\mathbf{p})\right)}{\kappa}}}{2 \sqrt{m^{2}+|\mathbf{p}|^{2}}} \hat{a}^{\dagger}(\mathbf{p}) e^{i S_{+}(\mathbf{p}) \cdot \mathbf{x}} e^{-i \omega^{+}(|\mathbf{p}|) x^{0}}+\int_{\mathbb{R}^{3}} \frac{\mathrm{d}^{3} p e^{\frac{\left.3 \omega^{-}(\mathbf{p})\right)}{\kappa}}}{2 \sqrt{m^{2}+|\mathbf{p}|^{2}}} \hat{b}(\mathbf{p}) e^{i S_{-}(\mathbf{p}) \cdot \mathbf{x}} e^{-i \omega^{-}(|\mathbf{p}|) x^{0}} \\
& +\int_{|\mathbf{p}|>\kappa} \frac{\mathrm{d}^{3} p e^{\frac{\left.3 \omega^{+}(\mathbf{p})\right)}{\kappa}}}{2 \sqrt{m^{2}+|\mathbf{p}|^{2}}} \hat{c}(\mathbf{p}) e^{i S_{+}(\mathbf{p}) \cdot \mathbf{x}} e^{-i \omega^{+}(|\mathbf{p}|) x^{0}}
\end{aligned}
$$


where

$$
\mathbf{S}_{ \pm}(\mathbf{p})=-e^{\frac{\omega^{ \pm}(\mathbf{p})}{\kappa}} \mathbf{p}
$$

Now we would like, in all of the three integrals in (56), to make the following change of variables:

$$
\mathbf{S}_{ \pm}(\mathbf{p})=\mathbf{q}
$$

The following relations:

$$
\mathbf{S}_{-}\left(\mathbf{S}_{+}(\mathbf{p})\right)=\mathbf{p} \quad \text { if }|\mathbf{p}|<\kappa, \quad \mathbf{S}_{+}\left(\mathbf{S}_{-}(\mathbf{p})\right)=\mathbf{p} \forall \mathbf{p} \in \mathbb{R}^{3}, \quad \mathbf{S}_{+}\left(\mathbf{S}_{+}(\mathbf{p})\right)=\mathbf{p} \quad \text { if }|\mathbf{p}|>\kappa,
$$

which are useful, respectively, in region $a, b$, and $c$ (See Fig. 6), allow us to invert $\mathbf{S}_{ \pm}(\mathbf{p})=\mathbf{q}$ for $\mathbf{p}$ :

$$
\mathbf{S}_{+}(\mathbf{p})=\mathbf{q} \Rightarrow\left\{\begin{array}{lll}
|\mathbf{p}|<\kappa & \mathbf{p}=\mathbf{S}_{-}(\mathbf{q}), & \mathbf{q} \in \mathbb{R}^{3}, \\
|\mathbf{p}|>\kappa & \mathbf{p}=\mathbf{S}_{+}(\mathbf{q}), & |\mathbf{q}|>\kappa,
\end{array} \quad \mathbf{S}_{-}(\mathbf{p})=\mathbf{q} \Rightarrow \mathbf{p}=\mathbf{S}_{+}(\mathbf{q}), \quad|\mathbf{q}|<\kappa\right.
$$

So in region $a$ we have to make the substitution $\mathbf{p}=\mathbf{S}_{-}(\mathbf{q})$, where now the integration domain for $\mathbf{q}$ is all of $\mathbb{R}^{3}$. In region $b$ we replace $\mathbf{p}=\mathbf{S}_{+}(\mathbf{q})$, while integrating $\mathbf{q}$ only in the region $|\mathbf{q}|<\kappa$. Finally, in $c$ too the transformation is $\mathbf{p}=\mathbf{S}_{+}(\mathbf{q})$, but the integration region is $|\mathbf{q}|>\kappa$. These transformations act on the frequencies in the following way:

$$
\omega^{-}\left(\mathbf{S}_{+}(\mathbf{q})\right)=-\omega^{+}(\mathbf{q}) \quad \text { if }|\mathbf{q}|<\kappa, \quad \omega^{+}\left(\mathbf{S}_{-}(\mathbf{q})\right)=-\omega^{-}(\mathbf{q}) \quad \forall \mathbf{q} \in \mathbb{R}^{3}, \quad \omega^{+}\left(\mathbf{S}_{+}(\mathbf{q})\right)=-\omega^{+}(\mathbf{q}) \quad \text { if }|\mathbf{q}|>\kappa .
$$

Again, the three relations above are useful, respectively, in region $a, b$, and $c$. Finally, the integration measure transforms in the following way:

$$
\frac{\mathrm{d}^{3} p}{2 \sqrt{m^{2}+|\mathbf{p}|^{2}}} \underset{\mathbf{p} \rightarrow \mathbf{S}_{ \pm}(\mathbf{q})}{\longrightarrow} \frac{\mathrm{d}^{3} q e^{\frac{-3 \omega^{ \pm}(|\mathbf{q}|)}{\kappa}}}{2 \sqrt{m^{2}+|\mathbf{q}|^{2}}}
$$

so, applying the coordinate transformations to the three integrals in (56), the expression for the Hermitian conjugate scalar field becomes

$$
\begin{aligned}
\hat{\phi}^{\dagger}(x)= & \int_{\mathbb{R}^{3}} \frac{\mathrm{d}^{3} q}{2 \sqrt{m^{2}+|\mathbf{q}|^{2}}} \hat{a}^{\dagger}\left(\mathbf{S}_{-}(\mathbf{q})\right) e^{i \mathbf{q} \cdot \mathbf{x}} e^{i \omega^{-}(|\mathbf{q}|) x^{0}}+\int_{|\mathbf{q}|<\kappa} \frac{\mathrm{d}^{3} q}{2 \sqrt{m^{2}+|\mathbf{q}|^{2}}} \hat{b}\left(\mathbf{S}_{+}(\mathbf{q})\right) e^{i \mathbf{q} \cdot \mathbf{x}} e^{i \omega^{+}(|\mathbf{q}|) x^{0}} \\
& +\int_{|\mathbf{q}|>\kappa} \frac{\mathrm{d}^{3} q}{2 \sqrt{m^{2}+|\mathbf{q}|^{2}}} \hat{c}\left(\mathbf{S}_{+}(\mathbf{q})\right) e^{i \mathbf{q} \cdot \mathbf{x}} e^{i \omega^{+}(|\mathbf{q}|) x^{0}} .
\end{aligned}
$$

\section{PAULI-JORDAN FUNCTION}

Scalar field theory quantization on $\kappa$-Minkowski was studied by several authors in the past. Among others, we believe the most remarkable approaches are the ones pursued in $[13,22,24,26]$. Each of those results contributed to our present understanding of field theory on $\kappa$-Minkowski. For example [13] was the first to consider the Green functions and the Pauli-Jordan function. Reference [24] exploited the de Sitter geometry of momentum space to define field theory in a Lorentz invariant way, and was the first to observe that the half-cover of de Sitter space offered by the bi-cross-product coordinates is sent to its complement by the elliptic identification map.
Early studies like [11,13] lacked the present understanding of the geometry of momentum space. This led to choices that we do not consider physical, e.g., in [13] the positive- and negative-frequency modes in the Pauli-Jordan function are distinguished by the sign of $p_{0}$, a choice that is not Lorentz covariant in our setting. In [11] on the other hand, the tower of complex solutions of the $\kappa$-deformed on-shell relation we discussed in Sec. III was considered a physical feature. This, as we argued in the preceding sections, is incompatible with momentum-space diffeomorphism invariance.

More recent studies, e.g., [24,26] take into account the de Sitter geometry of momentum space; however, they made use of Hamiltonian/canonical methods which are not 
explicitly spacetime covariant. Expressions involving onlyspace integrals are hard to make sense in $\kappa$-Minkowski, turning out to be basis dependent. In general one can note that $\kappa$-Minkowski commutation rules imply uncertainty relations of the kind:

$$
\delta t \delta x \sim \frac{1}{2 \kappa}\langle x\rangle,
$$

thus choosing a state on the algebra (9) such that $\delta t=0$ necessarily sets $\langle x\rangle=0$. As a consequence, defining equaltime canonical commutation rules such as

$$
[\dot{\phi}(0, \mathbf{x}), \phi(0, \mathbf{y})]=-i \delta^{3}(\mathbf{x}-\mathbf{y})
$$

is an ill-defined procedure.

An approach that is devoid of any of the problems listed above is [22,23] and following works [21,27,29]. These papers use a covariant symplectic form to define the canonical structure of the theory. This, being defined on the space of solutions, does not require a hypersurface of simultaneity or space-only integrals. The problem of the nonclosure of the region " $b$ " under Lorentz transformations is solved, in [22], by restricting the Hilbert space to the " $a$ " region, and demanding reality conditions on the field. This was extended to a complex field in [26], where the nonclosure of region $b$ under Lorentz transformations was solved by defining the Lorentz transformations in region $b$ as the image, under antipode, of the Lorentz transformations on region $a$ (which, we recall, leaves region $a$ closed).

In the present paper, we will follow a different approach, motivated by the request of invariance under coordinate transformations in momentum space. This immediately excludes the possibility of using Hamiltonian/canonical quantization methods, which rely on splitting time and 3-dimensional hypersurfaces of simultaneity. We have to rely on a covariant quantization scheme. In order to implement our momentum-space general coordinate invariance, we find the best-suited approach is to define the Pauli-Jordan function, an approach already explored in [13] (without however attempting to implement coordinate invariance).

In the commutative case, the covariant scalar field commutators $[\hat{\phi}(x), \hat{\phi}(y)]=i \Delta_{\mathrm{PJ}}(x, y)$ are given by

$$
\begin{aligned}
& i \Delta_{\mathrm{PJ}}(x, y)=i \Delta_{\mathrm{PJ}}(x-y) \\
& \quad=\int \frac{\mathrm{d}^{3} p}{2 \sqrt{\mathbf{p}^{2}+m^{2}}}\left(e^{i p \cdot(x-y)}-e^{-i p \cdot(x-y)}\right) \\
& \quad=\int \mathrm{d}^{4} p \operatorname{sign}\left(p_{0}\right) \delta\left(p^{2}-m^{2}\right) e^{i p \cdot(x-y)},
\end{aligned}
$$

and the $\Delta_{\mathrm{PJ}}$ function satisfies

$$
\left(\square_{x}+m^{2}\right) \Delta_{\mathrm{PJ}}(x, y)=\left(\square_{y}+m^{2}\right) \Delta_{\mathrm{PJ}}(x, y)=0 .
$$

Moreover $\Delta_{\mathrm{PJ}}$ is antisymmetric in its two variables, $\Delta_{\mathrm{PJ}}(y, x)=-\Delta_{\mathrm{PJ}}(x, y)$, and it is zero outside of the light cone:

$$
\Delta_{\mathrm{PJ}}(x, y)=0 \quad \text { if }(x-y)^{2}>0 .
$$

This last property is the most interesting one from the physical point of view, because it allows us to introduce a notion of light cone in a purely field-theoretical fashion.

We want to generalize $\Delta_{\mathrm{PJ}}$ to $\kappa$-Minkowski. First of all we need a generalization of the notion of bilocal function. The only such generalization we are aware of was introduced in [60]: a function of two points is assumed to be an element of $\mathcal{A} \otimes \mathcal{A}$. The basis elements of the algebra $\mathcal{A} \otimes \mathcal{A}$ generalize the notion of two independent points $z$ and $y$ :

$$
z^{\mu}=x^{\mu} \otimes \mathbb{1}, \quad y^{\mu}=\mathbb{1} \otimes x^{\mu} .
$$

With this construction we can generalize the properties (67) when $\Delta_{\mathrm{PJ}}(z, y) \in \mathcal{A} \otimes \mathcal{A}$ :

$$
\begin{aligned}
& \left(\left(\mathcal{C}_{\kappa}+m^{2}\right) \otimes \mathrm{id}\right) \triangleright \Delta_{\mathrm{PJ}}(z, y) \\
& \quad=\left(\mathrm{id} \otimes\left(\mathcal{C}_{\kappa}+m^{2}\right)\right) \triangleright \Delta_{\mathrm{PJ}}(z, y)=0 .
\end{aligned}
$$

The following expression satisfies the condition above and generalizes the Pauli-Jordan function in a way that is diffeomorphism invariant on momentum space:

$$
\begin{aligned}
i \Delta_{\mathrm{PJ}}(z, y)= & \frac{1}{2 m} \int \mathrm{d}^{4} p \sqrt{-g(p)} \varepsilon(p) \sqrt{-g^{\mu \nu}(p) \frac{\partial \mathcal{C}_{\kappa}}{\partial p^{\mu}} \frac{\partial \mathcal{C}_{\kappa}}{\partial p^{\nu}}} \\
& \times \delta\left(\mathcal{C}_{\kappa}+m^{2}\right): e^{i p_{\mu} z^{\mu}} e^{i S(p)_{\mu} y^{\mu}}:
\end{aligned}
$$

where : : refers to time-to-the-right ordering, and

$$
\varepsilon(p)= \begin{cases}+1 & \text { in region } a \\ -1 & \text { in region } b \\ -1 & \text { in region } c\end{cases}
$$

The form of the $\varepsilon$ function has been chosen in order to be invariant under the Lorentz flow, which connects regions $b$ and $c$. The function $\Delta_{\mathrm{PJ}}$ introduced here is also invariant under $\kappa$-Poincare transformations, understood in the following sense: $\quad z^{\mu} \rightarrow \Lambda^{\mu}{ }_{\nu} \otimes z^{\nu}+a^{\mu} \otimes \mathbb{1} \otimes \mathbb{1}, \quad y^{\mu} \rightarrow \Lambda^{\mu}{ }_{\nu} \otimes$ $y^{\nu}+a^{\mu} \otimes \mathbb{1} \otimes \mathbb{1}$, that is

$z^{\mu}=x^{\mu} \otimes \mathbb{1} \rightarrow \Lambda^{\mu}{ }_{\nu} \otimes x^{\nu} \otimes \mathbb{1}+a^{\mu} \otimes \mathbb{1} \otimes \mathbb{1}$,

$y^{\mu}=\mathbb{1} \otimes x^{\mu} \rightarrow \Lambda_{\nu}^{\mu} \otimes \mathbb{1} \otimes x^{\nu} \otimes \mathbb{1}+a^{\mu} \otimes \mathbb{1} \otimes \mathbb{1}$.

We can show that the exponentials $: e^{i p_{\mu} z^{\mu}} e^{i S(p)_{\mu} y^{\mu}}:$ transform in the following way:

$$
\begin{aligned}
& e^{i p_{i} z^{i}} e^{i p_{0} z^{0}} e^{i S(p)_{i} y^{i}} e^{i S(p)_{0} y^{0}} \\
& \quad \rightarrow e^{i \lambda_{i}[\xi, p] \otimes z^{i}} e^{i \lambda_{0}[\xi, p] \otimes z^{0}} e^{i S\left[\lambda_{i}[\xi, p]\right] \otimes y^{i}} e^{i S\left[\lambda_{0}[\xi, p]\right] \otimes y^{0}},
\end{aligned}
$$


where $\lambda_{\mu}[\xi, p]$ is the $3+1$-D generalization of the Lorentz transformation of momenta (28). The expression (71) is invariant under Lorentz transformations, so changing variables $p_{\mu} \rightarrow \lambda_{\mu}[\xi, p]$ allows us to prove that

$$
\begin{aligned}
& \Delta_{\mathrm{PJ}}(\Lambda \otimes z+a \otimes \mathbb{1} \otimes \mathbb{1}, \Lambda \otimes y+a \otimes \mathbb{1} \otimes \mathbb{1}) \\
& \quad=\mathbb{1} \otimes \Delta_{\mathrm{PJ}}(z, y),
\end{aligned}
$$

i.e., our noncommutative Pauli-Jordan function is $\kappa$ Poincaré invariant.

Solving the delta function in (71) we get

$$
\begin{aligned}
i \Delta_{\mathrm{PJ}}(z, y)= & \int_{|\mathbf{P}|<\kappa} \mathrm{d}^{3} p \frac{e^{3 \frac{\omega}{\alpha}^{+}} e^{i \mathbf{p} \cdot \mathbf{z}-i e^{\frac{\omega^{+}}{\kappa}} \mathbf{p} \cdot \mathbf{y}} e^{i \omega^{+}\left(z^{0}-y^{0}\right)}}{2 \sqrt{\left(m^{2}+|\mathbf{p}|^{2}\right)}} \\
& -\int_{\mathbb{R}^{3}} \mathrm{~d}^{3} p \frac{e^{3 \frac{\omega^{-}}{\kappa}} e^{i \mathbf{p} \cdot \mathbf{z}} e^{-i e^{\frac{\omega^{-}}{\kappa}} \mathbf{p} \cdot \mathbf{y}} e^{i \omega^{-}\left(z^{0}-y^{0}\right)}}{2 \sqrt{\left(m^{2}+|\mathbf{p}|^{2}\right)}} \\
& -\int_{|\mathbf{P}|>\kappa} \mathrm{d}^{3} p \frac{e^{3 \frac{\omega^{+}}{\kappa}} e^{i \mathbf{p} \cdot \mathbf{z}} e^{-i e^{\frac{\omega^{+}}{\kappa}} \mathbf{p} \cdot \mathbf{y}} e^{i \omega^{+}\left(z^{0}-y^{0}\right)}}{2 \sqrt{\left(m^{2}+|\mathbf{p}|^{2}\right)}} .
\end{aligned}
$$

The Pauli-Jordan-like function we defined is Poincaré invariant but it is not Hermitian nor antisymmetric under exchange of $z$ and $y$. However it satisfies the following conjugacy relation:

$$
\Delta_{\mathrm{PJ}}^{\dagger}(z, y)=\Delta_{\mathrm{PJ}}(y, z)
$$

because

$$
\left(: e^{i p_{\mu} z^{\mu}} e^{i S(p)_{\mu} y^{\mu}}:\right)^{\dagger}=: e^{i S(p)_{\mu} z^{\mu}} e^{i p_{\mu} y^{\mu}}:=: e^{i p_{\mu} y^{\mu}} e^{i S(p)_{\mu} z^{\mu}}: .
$$

This property is exactly what we expect from a field commutator:

$$
\left(\left[\hat{\phi}(z), \hat{\phi}^{\dagger}(y)\right]\right)^{\dagger}=\left[\hat{\phi}(y), \hat{\phi}^{\dagger}(z)\right] .
$$

\section{A. Covariant commutation relations}

Consider now the following commutation relations:

$$
\begin{aligned}
{\left[\hat{\phi}(z), \hat{\phi}^{\dagger}(y)\right] } & =i \Delta_{\mathrm{PJ}}(z, y), \\
{[\hat{\phi}(z), \hat{\phi}(y)] } & =\left[\hat{\phi}^{\dagger}(z), \hat{\phi}^{\dagger}(y)\right]=0,
\end{aligned}
$$

where of course we interpret $\hat{\phi}(z)$ as $\hat{\phi}(x) \otimes \mathbb{1}$ and $\hat{\phi}(y)$ as $\mathbb{1} \otimes \hat{\phi}(x)$. We can deduce the corresponding commutation relations for the creation and annihilation operators, $\hat{a}(\mathbf{p})$, $\hat{b}(\mathbf{p}), \hat{c}(\mathbf{p}), \hat{a}^{\dagger}(\mathbf{p}), \hat{b}^{\dagger}(\mathbf{p}), \hat{c}^{\dagger}(\mathbf{p})$, by replacing the expression (55) for $\hat{\phi}(z)$ and (63) for $\hat{\phi}^{\dagger}(y)$ into (80). A detailed calculation is presented in Appendix B. The final result is

$$
\begin{aligned}
& {\left[\hat{a}(\mathbf{p}), \hat{a}^{\dagger}(\mathbf{k})\right]=2 e^{-\frac{3 \omega^{+}(\mathbf{k})}{\kappa}} \sqrt{m^{2}+\left|\mathbf{S}_{+}(\mathbf{k})\right|^{2}} \delta^{(3)}[\mathbf{p}-\mathbf{k}],} \\
& {\left[\hat{b}(\mathbf{p}), \hat{b}^{\dagger}(\mathbf{k})\right]=2 e^{-\frac{3 \omega^{-}(\mathbf{k})}{\kappa}} \sqrt{m^{2}+\left|\mathbf{S}_{-}(\mathbf{k})\right|^{2}} \delta^{(3)}[\mathbf{p}-\mathbf{k}],} \\
& {\left[\hat{c}(\mathbf{p}), \hat{c}^{\dagger}(\mathbf{k})\right]=2 e^{-\frac{3 \omega^{+}(\mathbf{k})}{\kappa}} \sqrt{m^{2}+\left|\mathbf{S}_{+}(\mathbf{k})\right|^{2}} \delta^{(3)}[\mathbf{p}-\mathbf{k}],}
\end{aligned}
$$

with all the other commutators vanishing.

Except for a momentum-dependent weight that is necessary to make it Lorentz invariant, the algebra above is a standard creation and annihilation operator algebra. One can check that the algebra (81) is covariant under $\kappa$ deformed Lorentz transformations (28). In fact the delta function transforms like the inverse of $\mathrm{d}^{3} p$, and $\mathrm{d}^{3} p / \sqrt{m^{2}+\left|S_{ \pm}(\mathbf{p})\right|^{2}}$ transforms like the inverse of the volume element $\sqrt{-g(p)}=e^{-\frac{3 \omega^{ \pm}(\mathbf{P})}{\kappa}}$. Therefore, if $\hat{a}(\mathbf{p})$, $\hat{b}(\mathbf{p}), \hat{c}(\mathbf{p}), \hat{a}^{\dagger}(\mathbf{k}), \hat{b}^{\dagger}(\mathbf{k})$, and $\hat{c}^{\dagger}(\mathbf{k})$ transform like scalar fields on momentum space, the algebra (81) is left invariant.

The algebra (81) suggests a natural form for the number operator:

$$
\begin{aligned}
\hat{N}= & \int_{|\mathbf{p}|<\kappa} \frac{\mathrm{d}^{3} p e^{\frac{3 \omega^{+}(\mathbf{p})}{\kappa}}}{2 \sqrt{m^{2}+\left|\mathbf{S}_{+}(\mathbf{p})\right|^{2}}} \hat{a}^{\dagger}(\mathbf{p}) \hat{a}(\mathbf{p}) \\
& +\int_{\mathbb{R}^{3}} \frac{\mathrm{d}^{3} p e^{\frac{3 \omega^{-}(\mathbf{p})}{\kappa}}}{2 \sqrt{m^{2}+\left|\mathbf{S}_{-}(\mathbf{p})\right|^{2}}} \hat{b}^{\dagger}(\mathbf{p}) \hat{b}(\mathbf{p}) \\
& +\int_{|\mathbf{p}|>\kappa} \frac{\mathrm{d}^{3} p e^{\frac{3 \omega^{+}(|\mathbf{q}|)}{\kappa}}}{2 \sqrt{m^{2}+\left|\mathbf{S}_{+}(\mathbf{p})\right|^{2}}} \hat{c}^{\dagger}(\mathbf{p}) \hat{c}(\mathbf{p}) ;
\end{aligned}
$$

its commutation relations with the creation and annihilation operators are perfectly standard:

$$
\begin{array}{lll}
{\left[\hat{N}, \hat{a}^{\dagger}(\mathbf{p})\right]=\hat{a}^{\dagger}(\mathbf{p}),} & {[\hat{N}, \hat{a}(\mathbf{p})]=-\hat{a}(\mathbf{p}),} \\
{\left[\hat{N}, \hat{b}^{\dagger}(\mathbf{p})\right]=\hat{b}^{\dagger}(\mathbf{p}),} & {[\hat{N}, \hat{b}(\mathbf{p})]=-\hat{b}(\mathbf{p}),} \\
{\left[\hat{N}, \hat{c}^{\dagger}(\mathbf{p})\right]=\hat{c}^{\dagger}(\mathbf{p}),} & {[\hat{N}, \hat{c}(\mathbf{p})]=-\hat{c}(\mathbf{p}),}
\end{array}
$$

and moreover $\hat{N}$ is explicitly Hermitian: $\hat{N}^{\dagger}=\hat{N}$. This implies that we can define the Fock space exactly like in the commutative case.

In the rest of the present paper we are interested in the consequence for causality of the Pauli-Jordan function, so we will not dwell on the consequences of the algebra (81). It is worth mentioning, however, that the construction of the Fock space implementing the right statistics, with (anti) symmetrized multiparticle states, is a particularly complex challenge in $\kappa$-Minkowski, and several papers discussed the issue, e.g., [61-64]. The issue was initially noticed in [12], and the first paper to propose a solution is the already mentioned [22], where a deformed creation and annihilation operator algebra was introduced, in which commuting two operators changes the momentum of the particles they 
create/annihilate. Such commutation rules are capable of reproducing the $\kappa$-Poincaré coproduct rules. However, if a standard/undeformed (anti)symmetrization rule is assumed when defining multiparticle states, these will not have a well-defined total momentum. A deformed statistics is necessary to define multiparticle states which are eigenstates of the total momentum. The (anti)symmetrization proposed in [22] however is not covariant, and cannot be applied to massive fields. These further issues were addressed in [27], where the difficulty of satisfying Lorentz covariance in such a $\kappa$-deformed Fock space is shown to reduce to the problem of finding closed form, allorder expression for the $\mathrm{R}$ matrix of $\kappa$-Poincaré, which is still lacking. In the case of deformations of the Poincare algebra which do admit a closed-form $\mathrm{R}$ matrix, however, the problem is solvable: e.g., in the case of the Lorentz double in $2+1$ dimensions [65], where the deformed Fock space is related to the phenomenon of "flux metamorphosis" of non-Abelian anyons.

\section{MINIMAL-UNCERTAINTY STATES ON THE $\kappa$-MINKOWSKI ALGEBRA}

We now turn to developing the machinery that will allow us to extract physical predictions from the nonommutative Pauli-Jordan function we introduced in Sec. IV. $\Delta_{\mathrm{PJ}}(z, y)$ in fact is not a traditional function of two variables. It is rather an element of the non-Abelian algebra $\mathcal{A} \otimes \mathcal{A}$, and as such does not admit a single numerical value. By introducing a representation of $\mathcal{A} \otimes \mathcal{A}, \Delta_{\mathrm{PJ}}(z, y)$ can be made into an operator on a Hilbert space $\mathcal{H}$, and then one could invoke Born's rule to argue that a measurement involving $\Delta_{\mathrm{PJ}}(z, y)$ will deliver one of its eigenvalues with probability given by the squared norm of the value of the wave function on that eigenvalue. Of course we are talking about the state of the background quantum geometry, and invoking Born's rule in this case is likely unwarranted, as we lack the whole interpretational edifice of standard quantum mechanics, which is based on a vast empirical basis. This situation is, however, the daily bread of the quantum gravity researcher, and we have to make do with the mathematical structures at our disposal, without the power of experimental guidance, for the moment. Therefore we resort to the structure that is more likely to provide a translation from a quantum operator, $\Delta_{\mathrm{PJ}}(z, y)$, into definite outcomes: the expectation value on a state of the Hilbert space $\mathcal{H}$. The logic is the following: we suppose that the underlying quantum geometry is in some unknown state that cannot be determined within our theory, and the observables that depend on $\Delta_{\mathrm{PJ}}(z, y)^{8}$ will on average give outcomes that are compatible with a commutative Pauli-Jordan function of value $\left\langle\Delta_{\mathrm{PJ}}(z, y)\right\rangle$. The first goal is then to introduce a representation of our noncommutative algebra (9).

\footnotetext{
${ }^{8}$ Which could be, e.g., the arrival time of a particle in a detector (see below).
}

In [66] the irreducible representations of the $\kappa$ Minkowski algebra $\mathcal{A}$ are constructed, ${ }^{9}$ and they involve (for simplicity let us consider the 1+1-dimensional case) representing $\hat{x}^{0}$ as a derivative operator, ${ }^{10}$ and $\hat{x}^{0}=-\frac{i}{\kappa} \frac{d}{d x}$ and $\hat{x}^{1}$ as (plus or minus) the exponential of a multiplicative operator, $\hat{x}^{1}= \pm \frac{e^{x}}{\kappa}$ (notice that the placement of the constant $\kappa$ is unambiguously fixed by the request that $\hat{x}^{\mu}$ have dimensions of length while the "pregeometric" variable $x$ is dimensionless). These two are the nondegenerate representations, and one must complete them with a degenerate representation in which $\hat{x}^{1}$ is in an eigenstate with eigenvalue zero, and $\hat{x}^{0}$ has a real spectrum. These three representations can be put together by representing instead $\hat{x}^{1}$ as a multiplicative operator (in this case we use the variable $q$ ) and $\hat{x}^{0}$ as a dilatation operator:

$$
\hat{x}^{0}=\frac{i}{\kappa} q \frac{\mathrm{d}}{\mathrm{d} q}, \quad \hat{x}^{1}=\frac{q}{\kappa}, \quad q \in \mathbb{R} .
$$

In this way we are simultaneously considering the positive, negative, and zero parts of the spectrum of $\hat{x}^{1}$. Indeed on any test function $f$ the commutation relations (9) are respected:

$$
\begin{aligned}
{\left[\hat{x}^{0}, \hat{x}^{1}\right] \triangleright f } & =\left[\frac{i}{\kappa} q \frac{\mathrm{d}}{\mathrm{d} q}, \frac{q}{\kappa}\right] f=\frac{i}{\kappa} q \frac{\mathrm{d}}{\mathrm{d} q}\left(\frac{q}{\kappa} f\right)-q \frac{i}{\kappa} \frac{q}{\kappa} \frac{\mathrm{d}}{\mathrm{d} q} f \\
& =i \frac{q}{\kappa^{2}} f=\frac{i}{\kappa} \hat{x}^{1} \triangleright f .
\end{aligned}
$$

The representation we have introduced, however, has a problem: if the Hilbert space of functions over which this representation acts is $\mathcal{L}^{2}(\mathbb{R})$ with inner product

$$
\left(\psi_{1}, \psi_{2}\right)=\int_{\mathbb{R}} \mathrm{d} q \psi_{1}^{*}(q) \psi_{2}(q), \quad \psi \in \mathcal{L}^{2},
$$

then $\hat{x}^{0}$ is not Hermitian. This can be fixed by shifting the representation as

$$
\frac{i}{\kappa} q \frac{\mathrm{d}}{\mathrm{d} q} \rightarrow \frac{i}{\kappa} q \frac{\mathrm{d}}{\mathrm{d} q}+\frac{i}{2 \kappa}
$$

which can always be done since our representation is defined up to an additive constant. Now

$$
\begin{aligned}
\left(\psi_{1}, \hat{x}^{0} \triangleright \psi_{2}\right) & =\int_{\mathbb{R}} \mathrm{d} q \psi_{1}^{*}(q)\left(\frac{i}{\kappa} q \frac{\mathrm{d}}{\mathrm{d} q}+\frac{i}{2 \kappa}\right) \psi_{2}(q) \\
& =\int_{\mathbb{R}} \mathrm{d} q\left(\left(\frac{i}{\kappa} q \frac{\mathrm{d}}{\mathrm{d} q}+\frac{i}{2 \kappa}\right) \psi_{1}(q)\right) \psi_{2}(q) \\
& =\left(\hat{x}^{0} \triangleright \psi_{1}, \psi_{2}\right),
\end{aligned}
$$

\footnotetext{
${ }^{9}$ Larger realizations have been considered in the literature, e.g., involving deformed phase spaces $[67,68]$.

${ }^{10}$ From now on noncommuting coordinates will be represented with a hat.
} 
as can be easily seen. The representation we introduced can be easily generalized to $3+1$ dimensions as

$\hat{x}^{0}=\frac{i}{\kappa} \sum_{i=1}^{3} q_{i} \frac{\mathrm{d}}{\mathrm{d} q_{i}}+\frac{3 i}{2 \kappa}, \quad \hat{x}^{i}=\frac{q^{i}}{\kappa} \quad q_{i} \in \mathbb{R}^{3}$,

but for the rest of the paper we will only consider, for simplicity, the $1+1$ dimensional case without losing anything essential.

\section{A. Semiclassical states}

As we said before, we think of the Hilbert space $\mathcal{H}$ as the set of states of the underlying quantum geometry. $\kappa$-Minkowski is supposed to be an effective description of matter fields propagating on a quantum gravity background. By its nature, an effective theory cannot calculate everything it depends on within its own framework: some input is needed. In our case this input is represented by the element of $\mathcal{H}$ which represents the quantum state of our noncommutative coordinates. Of the infinitely many such states present in $\mathcal{H}$, many represent very nonclassical situations: one could have a superposition of macroscopically distant points (i.e., the wave function of $\hat{x}^{1}$ is peaked around points that are far away). Or the uncertainty on one of the coordinates could be enormous. If the underlying quantum theory of gravity that is supposed to admit $\kappa$ Minkowski as "ground state" produces such queer states, then we could probably rule it out with the observation that we experience, within a very small error margin, classical Minkowski spacetime as the geometric background on which known physics unfolds. Then we can, on physical ground, rule out most states in $\mathcal{H}$ as unphysical and focus on those that resemble more closely a classical geometry. We then formulate the following semiclassicality conditions for states in $\mathcal{H}$ : (1) the wave function needs to be localized, in the sense that the amplitudes need to fall off fast at a few variances away from the expectation values; (2) none of the variances of the time and spatial coordinates should be too large. This can be achieved by requiring that the squared sum of the variances $\left(\delta x^{0}\right)^{2}+\left(\delta x^{1}\right)^{2}$ is near its theoretical minimum. These variances are constrained by the uncertainty relations:

$$
\delta x^{0} \delta x^{1} \geq \frac{\left\langle x^{1}\right\rangle}{2 \kappa}
$$

It is clear that the values of $\delta x^{0}$ and $\delta x^{1}$ that minimize the squared sum $\left(\delta x^{0}\right)^{2}+\left(\delta x^{1}\right)^{2}$ are those such that ${ }^{11}$

\footnotetext{
${ }^{11}$ Notice that measurability limits of the form (91) have been already conjectured in [69], based on independent physical arguments that did not involve at all commutation relations of the form of $\kappa$-Minkowski.
}

$$
\delta x^{0} \sim \delta x^{1} \sim \sqrt{\frac{\left\langle x^{1}\right\rangle}{2 \kappa}}
$$

Within the above constraint, we still have at our disposal a vast class of wave functions that have good semiclassical properties. Only a more fundamental theory can single out a particular function, so, if we are working within an effective field theory framework, we should stay agnostic with regards to the particular choice of function, and study the behavior of our observables on all semiclassical wave functions. Universal features, i.e., features that are independent of the choice of function, can be deemed "emergent" properties of the theory, and can be legitimately claimed to be physical. In the following, we will consider Gaussian wave functions, which allow us to perform almost all calculations analytically, and impose the semiclassicality conditions within this restricted class of functions.

Consider a Gaussian state $|\psi\rangle$ with wave function

$\psi\left(q ;\left\langle x^{0}\right\rangle,\left\langle x^{1}\right\rangle\right) \equiv \psi(q)=\left(\frac{2}{\pi \sigma^{2}}\right)^{\frac{1}{4}} e^{-\frac{\left(q-\kappa\left\langle x^{1}\right\rangle\right)^{2}}{\sigma^{2}}-i \frac{\left\langle x^{0}\right\rangle}{\left.x^{1}\right\rangle} q}$.

$\left\langle x^{0}\right\rangle$ and $\left\langle x^{1}\right\rangle$ here are numerical parameters. Its $\mathcal{L}^{2}$ norm is 1 and the expectation values $\left\langle\psi\left|\hat{x}^{0}\right| \psi\right\rangle$ and $\left\langle\psi\left|\hat{x}^{1}\right| \psi\right\rangle$ are

$$
\begin{aligned}
& \left\langle\hat{x}^{0}\right\rangle=\int_{\mathbb{R}} \mathrm{d} q \psi^{*}(q)\left(\frac{i}{\kappa} q \frac{\mathrm{d}}{\mathrm{d} q}+\frac{i}{2 \kappa}\right) \psi(q)=\left\langle x^{0}\right\rangle, \\
& \left\langle\hat{x}^{1}\right\rangle=\int_{\mathbb{R}} \mathrm{d} q \psi^{*}(q) \frac{q}{\kappa} \psi(q)=\left\langle x^{1}\right\rangle .
\end{aligned}
$$

The variances are

$$
\begin{aligned}
\left(\delta \hat{x}^{0}\right)^{2} & =\left\langle\left(\hat{x}^{0}\right)^{2}\right\rangle-\left\langle\hat{x}^{0}\right\rangle^{2} \\
& =\left(\frac{\left\langle x^{1}\right\rangle}{\sigma}\right)^{2}+\frac{1}{4 \kappa^{2}}\left(2+\left(\frac{\left\langle x^{0}\right\rangle \sigma}{\left\langle x^{1}\right\rangle}\right)^{2}\right), \\
\left(\delta \hat{x}^{1}\right)^{2} & =\left\langle\left(\hat{x}^{1}\right)^{2}\right\rangle-\left\langle\hat{x}^{1}\right\rangle^{2}=\frac{\sigma^{2}}{4 \kappa^{2}} .
\end{aligned}
$$

We can find an optimal balance between the uncertainty in $\hat{x}^{0}$ and that in $\hat{x}^{1}$ by minimizing the sum $\left(\delta \hat{x}^{0}\right)^{2}+\left(\delta \hat{x}^{1}\right)^{2}$

$$
\begin{aligned}
& \frac{\mathrm{d}}{\mathrm{d} \sigma}\left(\left(\delta \hat{x}^{0}\right)^{2}+\left(\delta \hat{x}^{1}\right)^{2}\right) \\
& \quad=\frac{\mathrm{d}}{\mathrm{d} \sigma}\left(\left(\frac{\left\langle x^{1}\right\rangle}{\sigma}\right)^{2}+\frac{1}{4 \kappa^{2}}\left(2+\left(\frac{\left\langle x^{0}\right\rangle \sigma}{\left\langle x^{1}\right\rangle}\right)^{2}\right)+\frac{\sigma^{2}}{4 \kappa^{2}}\right)=0 .
\end{aligned}
$$

This equation selects a specific value of $\sigma$ :

$$
\sigma^{4}=\frac{4 \kappa^{2}\left\langle x^{1}\right\rangle^{4}}{\left\langle x^{0}\right\rangle^{2}+\left\langle x^{1}\right\rangle^{2}}
$$


Close to the classical light cone, i.e., when $\left\langle\hat{x}^{0}\right\rangle \sim\left\langle\hat{x}^{1}\right\rangle$, we have $\sigma^{2} \sim 2 \kappa\left\langle x^{1}\right\rangle$, and the uncertainties are $\left(\delta \hat{x}^{0}\right)^{2} \sim \frac{3\left\langle x^{1}\right\rangle}{2 \sqrt{2} \kappa}$, $\left(\delta \hat{x}^{1}\right)^{2} \sim \frac{\left\langle x^{1}\right\rangle}{2 \sqrt{2} \kappa}$.

Let us look into some real-world numbers, and imagine we are considering an astrophysical event, e.g., a gamma ray burst which is highly localized in space and time, compared to the space and time distances at which the event is located (gamma ray bursts can be located as far back in time as several billion years, and their temporal resolution can be of the order of the second). Assuming that the deformation parameter $\kappa$ is equal to the Planck energy, $\kappa \sim E_{p} \sim 10^{28} \mathrm{eV}$, then its inverse is (with the appropriate powers of $\hbar$ and $c$, which we omit) the Planck length, $\frac{1}{\kappa} \sim L_{p} \sim 10^{-35} \mathrm{~m}$. Now consider a point near the classical light cone, at 2 billion light years from the origin, i.e., the expectation value of $\hat{x}^{1}$ is $\left\langle\hat{x}^{1}\right\rangle \sim 2 \times 10^{9} \mathrm{ly}$, or, in terms of the Planck length, $\left\langle\hat{x}^{1}\right\rangle \sim 10^{60} L_{p}$. Our semiclassical Gaussian state in this case would have

$$
\begin{aligned}
& \delta \hat{x}^{1} \sim \sqrt{\frac{\left\langle x^{1}\right\rangle L_{p}}{2 \sqrt{2}}} \sim 6 \times 10^{-6} \mathrm{~m}, \\
& \delta \hat{x}^{0} \sim \sqrt{3 \frac{\left\langle x^{1}\right\rangle L_{p}}{2 \sqrt{2}}} \sim 3 \times 10^{-14} \mathrm{~s} .
\end{aligned}
$$

So we can prepare a semiclassical state of one point centered on $\left\langle\hat{x}^{1}\right\rangle \sim 2$ billion light years, $\left\langle\hat{x}^{0}\right\rangle \sim 2$ billion years, and $\delta \hat{x}^{0} \sim 30$ femtoseconds, $\delta \hat{x}^{1} \sim 6 \mu \mathrm{m}$. This state has a microscopic space and time uncertainty while the expectation values are of the order of billion (light) years.

Now, if the expectation value of the Pauli-Jordan function on such a geometric state has a tail outside of the classical light cone $\left\langle\hat{x}^{1}\right\rangle=\left\langle\hat{x}^{0}\right\rangle$ that is exponentially suppressed over a range of the order of the 10 femtoseconds (or tens of $\mu \mathrm{m}$ ), we can say that all the fuzziness of the light cone is due to the intrinsic uncertainty of the geometric state, and there is no effect due to propagation over cosmological distances. Instead, if it is suppressed over a much greater range (e.g., over time intervals of the order of $1 \mathrm{sec}$, as is necessary in order to reveal such effects with astrophysical sources like gamma ray bursts [43]), we can say that we expect photons or neutrinos from distant localized sources to arrive with a measurable uncertainty in the time of arrival, whose origin is to be traced back to the quantum fluctuations of the underlying spacetime. We now turn to evaluating the Pauli-Jordan function on a semiclassical Gaussian state of two coordinates $\hat{y}^{\mu}$ and $\hat{z}^{\mu}$, which will allow us to study its dependence on the four free parameters of the state: the expectation values $\left\langle y^{0}\right\rangle,\left\langle z^{0}\right\rangle$, $\left\langle y^{1}\right\rangle$, and $\left\langle z^{1}\right\rangle$.

\section{B. Expectation value of the Pauli-Jordan function around the light cone}

We now consider a representation of the $(1+1$ dimensional version of the) tensor product algebra (9):

$\begin{array}{rlrl}\hat{z}^{0} & =\frac{i}{\kappa} q_{z} \frac{\mathrm{d}}{\mathrm{d} q_{z}}+\frac{i}{2 \kappa}, & \hat{z}^{1}=\frac{q_{z}}{\kappa}, & q_{z} \in \mathbb{R}, \\ \hat{y}^{0}=\frac{i}{\kappa} q_{y} \frac{\mathrm{d}}{\mathrm{d} q_{y}}+\frac{i}{2 \kappa}, & \hat{y}^{1}=\frac{q_{y}}{\kappa}, & q_{y} \in \mathbb{R} .\end{array}$

We are interested in calculating matrix elements such as $\left\langle\psi_{z}, \psi_{y}\left|e^{i k \hat{z}} e^{i \omega^{+}(k) \hat{z}^{0}}\right| \psi_{z}, \psi_{y}\right\rangle$, where

$$
\begin{aligned}
& \Delta_{\mathrm{PJ}}(\hat{z}, \hat{y})=\int_{|p|<\kappa} \mathrm{d} p \frac{e^{\frac{\omega^{+}}{\kappa}} e^{i p \hat{z}-i e^{\frac{\omega^{+}}{\kappa}} p \hat{y}} e^{i \omega^{+}\left(\hat{z}^{0}-\hat{y}^{0}\right)}}{2 \sqrt{m^{2}+p^{2}}} \\
& -\int \mathrm{d} p \frac{e^{\frac{\omega^{-}}{\kappa}} e^{i p \hat{z}} e^{-i e^{\frac{\omega^{-}}{\kappa}} p \hat{y}} e^{i \omega^{-}\left(\hat{z}^{0}-\hat{y}^{0}\right)}}{2 \sqrt{m^{2}+p^{2}}} \\
& -\int_{|p|>\kappa} \mathrm{d} p \frac{e^{\omega^{\omega^{+}}} e^{i p \hat{z}} e^{-i e^{\frac{\omega^{+}}{\kappa}} p \hat{y}} e^{i \omega^{+}\left(\hat{z}^{0}-\hat{y}^{0}\right)}}{2 \sqrt{m^{2}+p^{2}}} \\
& =: \Delta_{a}(\hat{z}, \hat{y})+\Delta_{b}(\hat{z}, \hat{y})+\Delta_{c}(\hat{z}, \hat{y}),
\end{aligned}
$$

and the states $\left|\psi_{z}, \psi_{y}\right\rangle$ are products of Gaussian states:

$$
\left|\psi_{z}, \psi_{y}\right\rangle=\psi\left(q_{z} ;\left\langle z^{0}\right\rangle,\left\langle z^{1}\right\rangle\right) \psi\left(q_{y} ;\left\langle y^{0}\right\rangle,\left\langle y^{1}\right\rangle\right) .
$$

Notice that the $\hat{z}^{0}$ operator is just the dilatation operator plus a phase shift:

$e^{i \omega^{+}(p) \hat{z}^{0}} \triangleright \psi\left(q_{z}\right)=e^{i \omega^{+}(p)\left(\frac{i}{\kappa} q_{z} \frac{\mathrm{d}}{\mathrm{d} q_{z}}+\frac{i}{2 \kappa}\right)} \psi\left(q_{z}\right)=e^{-\frac{\omega^{+}(p)}{2 \kappa}} \psi\left(e^{-\frac{\omega^{+}}{\kappa}} q_{z}\right)$,

while $e^{i p \hat{z}^{1}} \triangleright \psi\left(q_{z}\right)=e^{i k q_{z}} \psi\left(q_{z}\right)$. In order to calculate, for instance, $\left\langle\psi_{z}, \psi_{y}\left|\Delta_{a}(\hat{z}, \hat{y})\right| \psi_{z}, \psi_{y}\right\rangle$ we need

$$
\begin{aligned}
f_{z}(p) & :=\left\langle\psi_{z}\left|e^{i p \hat{z}^{1}} e^{i \omega^{+}(p) \hat{z}^{0}}\right| \psi_{z}\right\rangle \\
& =\int_{\mathbb{R}} \mathrm{d} q_{z} \psi^{*}\left(q_{z}\right) e^{i p q_{z}} e^{-\frac{\omega^{+}(p)}{2 \kappa}} \psi\left(e^{-\frac{\omega^{+}}{\kappa}} q_{z}\right),
\end{aligned}
$$

and

$$
\begin{aligned}
f_{y}(p) & :=\left\langle\psi_{y}\left|e^{-i p \hat{y} 1} e^{\frac{\omega^{+}(p)}{\kappa}} e^{-i \omega^{+}(p) \hat{y}^{0}}\right| \psi_{y}\right\rangle \\
& =\int_{\mathbb{R}} \mathrm{d} q_{y} \psi^{*}\left(q_{y}\right) e^{-i p q_{y} e^{\frac{\omega^{+}(p)}{\kappa}}} e^{\frac{\omega^{+}(p)}{2 \kappa}} \psi\left(e^{\frac{\omega^{+}}{\kappa}} q_{y}\right),
\end{aligned}
$$

which are Gaussian integrals and can be readily calculated analytically, with parameters $\left\langle z^{0}\right\rangle,\left\langle z^{1}\right\rangle,\left\langle y^{0}\right\rangle,\left\langle y^{1}\right\rangle, m, \kappa$ (and, if we do not want to specialize immediately to semiclassical Gaussian states, also the variances $\sigma_{z}$ and $\sigma_{y}$ ). After that one can evaluate numerically 


$$
\left\langle\psi_{z}, \psi_{y}\left|\Delta_{\mathrm{PJ}}(\hat{z}, \hat{y})\right| \psi_{z}, \psi_{y}\right\rangle=\int_{-\kappa}^{\kappa} \mathrm{d} p \frac{e^{\frac{\omega^{+}}{\kappa}}}{2 \sqrt{p^{2}+m^{2}}} f_{z}(p) f_{y}(p),
$$

where the $f$ are some functions of momenta and parameters. An analog procedure allows us to calculate the expectation values of $\Delta_{b}(\hat{z}, \hat{y})$ and $\Delta_{c}(\hat{z}, \hat{y})$ on $\left|\psi_{z}, \psi_{y}\right\rangle$.

Our calculation reveals that $\Delta_{\mathrm{PJ}}$ is real (or at least its imaginary part is smaller than the numerical error), just like in the commutative case. Moreover, when $\left\langle z^{\mu}\right\rangle-\left\langle y^{\nu}\right\rangle$ is spacelike the noncommutative Pauli-Jordan function is not zero; however, it falls off exponentially away from the light cone, with a characteristic length of the order of $\sqrt{L_{p}\left\langle z^{1}\right\rangle}$. We conclude that the light cone in $\kappa$-Minkowski spacetime is "blurry," and "spills out" of the classical light cone over a distance of the same order of magnitude of the minimal intrinsic uncertainty of semiclassical states on the underlying noncommutative geometry. For a pointlike source placed at a distance $\left\langle z^{1}\right\rangle$ of the order of one billion light years, one can expect photons to arrive with an uncertainty of the order of $\sqrt{L_{p}\left\langle z^{1}\right\rangle} \sim 10^{-14} s=10 \mathrm{fs}$, but not more. This is 14 orders of magnitude less than that predicted by a linear relation of the kind (1) for $E=1 \mathrm{TeV}$ particles.

\section{OUTLOOK AND CONCLUSIONS}

Our approach allowed us to define a quantum noninteracting scalar field theory on the $\kappa$-Minkowski noncommutative spacetime, in a way that is (1) completely covariant, (2) invariant under diffeomorphism of momentum space, and (3) $\kappa$-Poincaré invariant.

Point (1) refers to the fact that our quantization prescription does not depend on 3-dimensional structures like $3+1$ decompositions of spacetime. Hamiltonian formulations or symplectic structures $[22,24,26]$ are inconsistent in $\kappa$-Minkowski due to its peculiar commutators $\left[x^{0}, x^{i}\right]=\frac{i}{\kappa} x^{i}$. These imply Heisenberg-like relations $\delta x^{0} \delta x^{i} \geq \frac{1}{2 \kappa}\left\langle x^{i}\right\rangle$ that do not allow the uncertainty on the time coordinate, $\delta x^{0}$, to be infinitely small: one therefore loses the notion of a spacelike hypersurface. Covariant commutators (81) and the Pauli-Jordan function allowed us to quantize the theory in a 4-dimensional way, without being forced to make reference to any hypersurface of simultaneity (and therefore without referring to a particular inertial frame). There is already in the literature (see $[13,11])$ an attempt at introducing Pauli-Jordan function (and the related Green functions). However, at the time, the understanding of $\kappa$-Minkowski's momentum space was somewhat limited, and this led the authors of $[11,13]$ to define their Green functions as contour integrals on the complexification of a particular timelike "energy" coordinate on momentum space. Complexifying this coordinate breaks diffeomorphism invariance and introduces an infinite tower of poles of the Green functions. The authors interpreted this as a physical feature of the theory. With hindsight, we can exclude that these features have physical meaning, because they depend on the choice of a particular set of coordinates on momentum space. This brings us to point (2), invariance under diffeomorphisms of momentum space. One of our basic principles is that only statements that are coordinate independent on momentum space should be physical. Without such an assumption, the nonlinear structures introduced by spacetime noncommutativity would make the theory not predictable. One could draw all sorts of mutually incompatible conclusions just by choosing different coordinate systems on momentum space. As observed in Sec. II [e.g., Eq. (14)] each ordering prescription for $\kappa$-Minkowski plane waves corresponds to a different coordinate system on momentum space. Moreover each of these coordinate system corresponds to a choice of basis for momentum generators in the $\kappa$-Poincaré algebra. Just like in a Lie algebra one is free to make linear redefinitions of the generators (without changing the algebra itself); in a Hopf algebra this freedom extends to any nonlinear redefinition of the generators. If we are to use Hopf algebras to describe the symmetries of a noncommutative spacetime, we should not break their basic invariance properties when building physical models on such a framework. Point (3) is of course the reasonable request that all of the equations of the theory, in particular the commutation relations, be left invariant by $\kappa$-Poincaré transformations. There is one subtlety in this point, something that in the commutative case one does not need to worry about. In the commutative case one can check independently the invariance under translations and Lorentz transformations; this ensures invariance under the whole Poincare group. In the noncommutative case one should look for invariance under a complete $\kappa$-Poincaré transformation of the form (10). Invariance under such a transformation does not imply invariance under a "pure" Lorentz transformation of the form $x^{\mu} \rightarrow \Lambda^{\mu}{ }_{\mu} \otimes x^{\nu}$. For example, to prove the $\kappa$-Poincare invariance of the PauliJordan function (75) we have to use the commutation relations $\left[a^{\mu}, \Lambda^{\rho}{ }_{\sigma}\right]$ [see Eq. (6)]. Setting $a^{\mu}$ to zero would not deliver the desired result: the Pauli-Jordan function is not invariant under "pure-Lorentz" transformations. This is an aspect of the so-called "no pure boost" property of the $\kappa$-Poincaré group (first noticed in [25]). The commutators (6) imply uncertainties between $\Lambda^{\mu}{ }_{\nu}$ and $a^{\mu}$ such that we cannot set $\delta a^{\mu}=0$, not without making either $\delta \Lambda^{\mu}{ }_{\nu}$ divergent or by setting the expectation $\left\langle\Lambda^{\mu}{ }_{\nu}\right\rangle=\delta^{\mu}{ }_{\nu}$ (at least for some components of $\Lambda_{\nu}^{\mu}$ ). This means that we cannot fix the translation parameters to be zero with total certainty, and still be free to choose the Lorentz parameters arbitrarily.

The most advanced theoretical result that was enabled by our approach is the definition of the creation and annihilation operators algebra (81), and the introduction of a number operator $\hat{N}$ with standard commutation rules (83). 
The number operator is Hermitian, and therefore we can construct a Fock space in the standard way, with a vacuum that is annihilated by all of the annihilation operators. The $n$-particle states are eigenstates of $\hat{N}$ with eigenvalue $n$, and are obtained by the successive application of creation operators.

The next step of our analysis would be to introduce an electric charge operator (recall that our scalar field is complex) and C, P, and T conjugation operators, and then to study how they are represented on the creation and annihilation operator algebra. In the commutative case the charge operator is just a number operator with a minus sign in front of the integral on negative-frequency states. By analogy it seems natural to introduce a noncommutative charge operator as the same expression as (82), with a minus in front of the second and third integral. Such an expression is Hermitian and $\kappa$-Poincaré invariant. However in this case we anticipate a difficulty with the definition of charge conjugation: if the antipode map is used to define the charge conjugation operator, then there is an issue with Lorentz covariance. In fact the antipode maps region $a$ to region $b$, and maps region $c$ onto itself. But Lorentz transformations connect regions $b$ and $c$ and this could lead to a non-Lorentz-invariant charge conjugation operator. These issues deserve a focused analysis, which we postpone to future studies.

On the level of physical interpretation and, possibly, phenomenology, our study focused on a prescription to extract observable features from our noncommutative Pauli-Jordan function. In particular we were interested in understanding how to generalize to our noncommutative setting the well-known result that the Pauli-Jordan function defines a light cone. In ordinary QFT this function is zero on spacelike intervals. To achieve this result we had to introduce a representation of the noncommutative algebra $\mathcal{A}$ of $\kappa$-Minkowski coordinates (9), and a Hilbert space $\mathcal{H}$ on which this representation acts. The Pauli-Jordan function is a function of two variables (i.e., an element of $\mathcal{A} \otimes \mathcal{A}$ ), and therefore we need to consider the tensor product Hilbert space $\mathcal{H} \otimes \mathcal{H}$. There are many states on this Hilbert space, and our theory is not able to make any prediction regarding which of those will be physically realized. We then make an ansatz: our field theory on $\kappa$ Minkowski is supposed to be an effective description of matter on a quantum gravity background, once the gravitational degrees of freedom are integrated away (in analogy to what happens in $2+1$ dimensions [1,2]). Then the particular state of the quantum geometry can only be determined by the underlying quantum gravity theory, which we do not have access to. The best we can do is to make an educated guess regarding the properties of the states that are physically realized. The one we made is based on the insight that such states will have to be "as classical as possible," resembling commutative, classical Minkowski geometry as much as possible. This excludes those states in $\mathcal{H}$ in which the uncertainty of one of the coordinates is macroscopically large, for instance. We therefore introduce a notion of "semiclassical" states which minimize the squared sum of the uncertainties of the coordinates, i.e., $\left(\delta \hat{x}^{0}\right)^{2}+\left(\delta \hat{x}^{1}\right)^{2}$ is minimal. Among those states (which are still uncountably many) we may pick one and calculate the expectation value of the Pauli-Jordan function $\left\langle\Delta_{\mathrm{PJ}}(\hat{z}, \hat{y})\right\rangle$. The result is a commutative function that depends on the state, and we are interested in how it varies when the expectation value of the coordinates of the two points, $\left\langle\hat{z}^{\mu}\right\rangle$ and $\left\langle\hat{y}^{\mu}\right\rangle$, are varied. These expectation values now are commutative functions and define a classical lightcone (i.e., when $\left\langle\hat{z}^{\mu}\right\rangle-\left\langle\hat{y}^{\mu}\right\rangle$ is lightlike). One can check whether $\left\langle\Delta_{\mathrm{PJ}}(\hat{z}, \hat{y})\right\rangle$ vanishes outside of this classical light cone, or it possesses a "tail." In the massless case it is easy to integrate the commutative PauliJordan function for a massless scalar field [Eq. (66)]-in the $1+1$ dimensional case-and get

$$
\begin{aligned}
\Delta_{\mathrm{PJ}}(z, y)= & \frac{\pi}{2} \operatorname{sign}\left[\left(z^{0}-y^{0}\right)-\left(z^{1}-y^{1}\right)\right] \\
& -\frac{\pi}{2} \operatorname{sign}\left[\left(z^{0}-y^{0}\right)+\left(z^{1}-y^{1}\right)\right] .
\end{aligned}
$$

We plot this function in the plane $\left(z^{1}-y^{1}\right)$ vs $\left(z^{0}-y^{0}\right)$ in Fig. 9. Its main feature is that it is zero on lightlike intervals,

$$
\kappa\left\langle z^{0}\right\rangle=10^{3}
$$

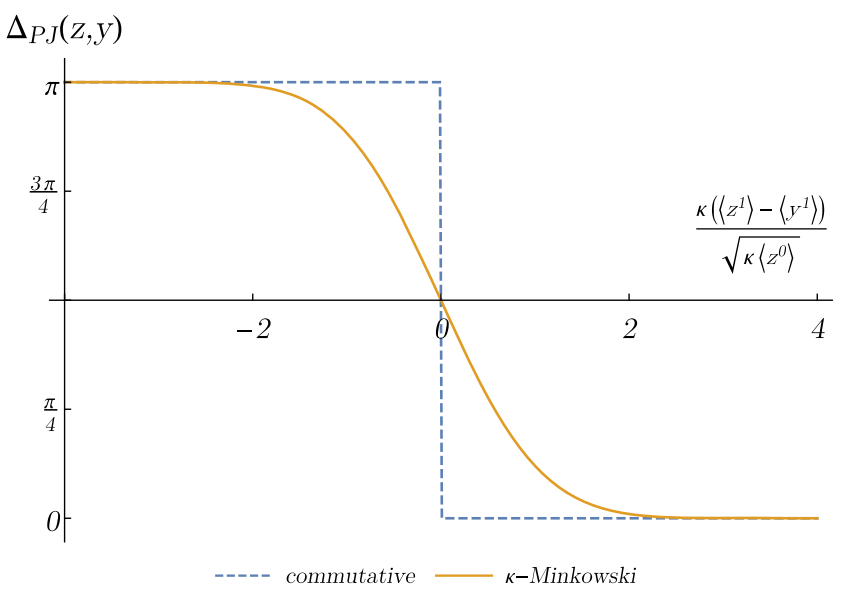

FIG. 7. Pauli-Jordan function for Minkowski (dashed line) and $\kappa$-Minkowski (solid line) spacetimes. The $\langle y\rangle$ coordinates are fixed to a smaller value $\left\langle y^{0}\right\rangle \sim\left\langle y^{1}\right\rangle \sim \frac{1}{100}\left\langle z^{0}\right\rangle$, while the $\left\langle z^{0}\right\rangle$ coordinate is fixed to a large value (in this case $10^{3}$ in units of $\left.\kappa^{-1}\right)$. The $\left\langle z^{1}\right\rangle$ coordinate varies on the horizontal axis around the light-cone value $\left\langle z^{1}\right\rangle=\left\langle z^{0}\right\rangle$, over an interval of a few units of $\sqrt{\left\langle z_{0}\right\rangle / \kappa}$. In this simulation we are looking at unrealistically small distances (1000 Planck lengths), but realistic values (i.e., $10^{9}$ light years $=10^{60}$ Planck lengths) are impossible to work with on the calculator. Our strategy is therefore to start with small distances, and then see how the result evolves as we increase the distances by several orders of magnitude at the time (see Fig. 8 below). 

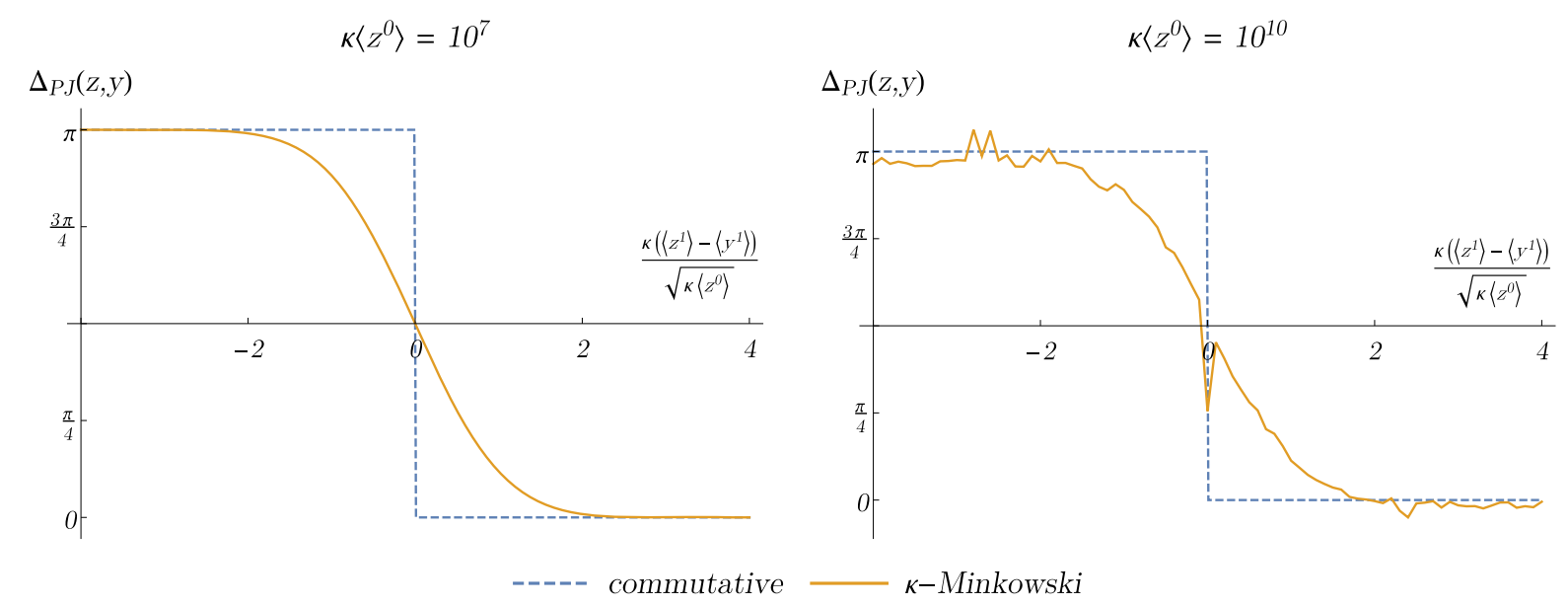

FIG. 8. The Pauli-Jordan function calculated at $10^{7}$ and $10^{10}$ Planck units of distance from $\left\langle y^{\mu}\right\rangle$. The horizontal scale is units of $\sqrt{\left\langle z^{0}\right\rangle / \kappa}$. We see that in this scale the function has exactly the same shape, independently of the value of $\kappa\left\langle z^{0}\right\rangle$ (the right-hand-side plot suffers from significant numerical errors, but it shows, near to the limits of our numerical precision, that the shape of the function is still the same). It is therefore safe to assume that the shape of the Pauli-Jordan function will be the same also for $\kappa\left\langle z^{0}\right\rangle \sim 10^{60} L_{p} \sim 1$ billion light years.

while it is $\pm \pi$ inside the light cone ( $+\pi$ in the future light cone and $-\pi$ in the past one). The noncommutative generalization of this function is the expectation value $\left\langle\Delta_{\mathrm{PJ}}(\hat{z}, \hat{y})\right\rangle$, which we plot in Fig. 10 (our numerical calculation revealed that $\left\langle\Delta_{\mathrm{PJ}}(\hat{z}, \hat{y})\right\rangle$ is real within the numerical error). The plots show how the border between the region where $\left\langle\Delta_{\mathrm{PJ}}(\hat{z}, \hat{y})\right\rangle$ is a nonzero constant and the region where it is zero gets blurred. The size of the region over which this blurring occurs depends on the distance from the origin: in the leftmost plot of Fig. 9 we are focusing on a region of size $20 \times 20$ Planck units $\kappa^{-1}(20$ Planck lengths $\times 20$ Planck times), and the blurring zone

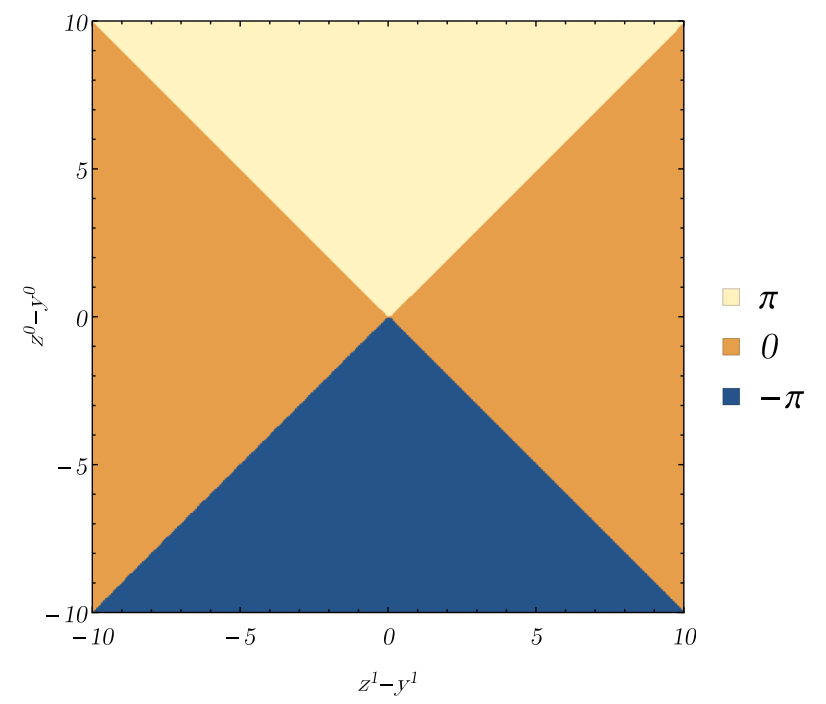

FIG. 9. Contour plot of the Pauli-Jordan function in the plane of space intervals $z^{1}-y^{1}$ vs time intervals $z^{0}-y^{0}$, for a commutative massless scalar field (units are arbitrary, as the plot is scale invariant). extends over several Planck units. The other two diagrams are zoomed out over, respectively, a $200 \times 200$ and a $2000 \times 2000$ Planck units region. The blurring zone in those two cases extends over order $\sim 10$ and order $\sim 30 \sim$ $\sqrt{1000}$ Planck units. We see that the blurring range goes like the square root of the distance from the origin in Planck units, or, in other words, as the geometric mean $\sqrt{L \kappa^{-1}}$ between the distance from the origin $L$ and the Planck scale $\kappa^{-1}$. This can be seen clearly in Figs. 7 and 8, where we plot a constant $-\left(\left\langle z^{0}\right\rangle-\left\langle y^{0}\right\rangle\right)$ slice of $\left\langle\Delta_{\mathrm{PJ}}(\hat{z}, \hat{y})\right\rangle$, centred upon the classical light cone, $\left(\left\langle z^{1}\right\rangle-\left\langle y^{1}\right\rangle\right)=\left(\left\langle z^{0}\right\rangle-\left\langle y^{0}\right\rangle\right)$, and rescaled by $\sqrt{\left\langle z^{0}\right\rangle \kappa^{-1}}$. The three diagrams represent three choices of $\sqrt{\left\langle z^{0}\right\rangle \kappa^{-1}}$ or $\sqrt{L \kappa^{-1}}: 10^{3}, 10^{7}$ and $10^{10}$, and it becomes apparent how, in these scales, the three slices of the Pauli-Jordan function appear exactly identical. Therefore, we can conclude that the blurring radius is always of order $\sqrt{L \kappa^{-1}}$.

To obtain our result we chose a particular example of "semiclassical" wave function: a Gaussian (92). This allows us to integrate analytically all of the functions that appear in $\left\langle\Delta_{\mathrm{PJ}}(\hat{z}, \hat{y})\right\rangle$, except the very last one, and permits us to produce particularly clean numerical results. The Gaussian wave function is characterized only by two parameters: the expectation values of the time and space coordinates (the normalization being fixed to one and the variance being fixed by the semiclassicality condition). It is with respect to these two variables that we study the variation of $\left\langle\Delta_{\mathrm{PJ}}(\hat{z}, \hat{y})\right\rangle$. In general, a wave function satisfying the semiclassicality condition will be much more complicated than (92), and will depend on a potentially infinite set of parameters. We conjecture that the dependence of $\left\langle\Delta_{\mathrm{PJ}}(\hat{z}, \hat{y})\right\rangle$ on all of these additional parameters will be weak, and the essential features of the function will 

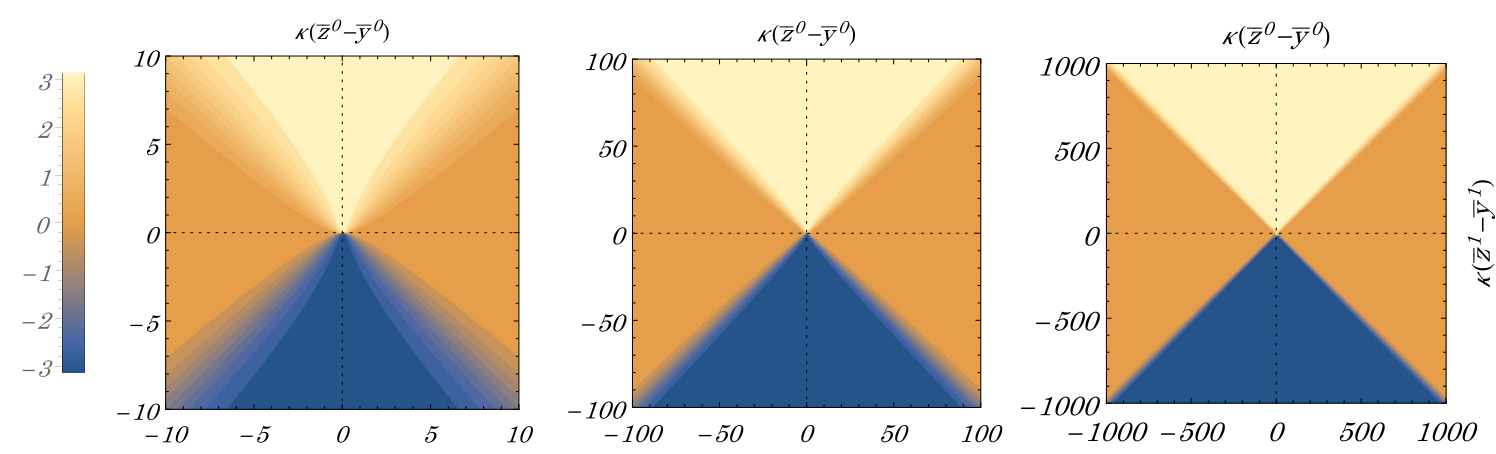

FIG. 10. Same diagram as Fig. 9 in the noncommutative case. The diagram on the left is zoomed in at the origin, and shows a region of $20 \times 20$ Planck units $\kappa^{-1}$. The central diagram shows $200 \times 200$ Planck units, while the one on the left shows $2000 \times 2000$.

only depend on the expectation values $\left\langle\hat{z}^{\mu}\right\rangle$ and $\left\langle\hat{y}^{\mu}\right\rangle$. We provide evidence to support this conjecture in [70], where we calculate the expectation value of the Pauli-Jordan function on three additional choices of wavefunction. We observe that the result depends only on the expectation values $\left\langle z^{\mu}\right\rangle$ and $\left\langle y^{\mu}\right\rangle$, and not on the details of the test wave functions, provided they satisfy the semiclassicality condition. This point will be further illustrated in [70].

For what concerns phenomenology, the shape of our Pauli-Jordan function leads us to the conclusion that there is an in-principle-observable violation of the classical light cone. The Pauli-Jordan function spills out of the classical light cone by an amount that depends on the geometric mean of the distance from the source and the Planck scale. This suggests that, on a $\kappa$-Minkowski background, a perfectly localized signal emitted at a distance $L$ will be detected with an uncertainty in time and space of the order of $\sqrt{L \kappa^{-1}}$. Real-life numbers are quite small: for $L \sim 1$ billion lightyears we get an uncertainty in the time of arrival of the order of 10 femtoseconds. If we had a sufficiently bright ultrashort pulse source (i.e., a femtosecond laser) placed at one billion years from Earth, we would be able to detect the effect as a broadening of the pulse duration upon detection. Similarly, a distant source that is sufficiently localized in space (i.e., a micrometer-scale source one billion lightyears away) would allow detection of our effect with present-day technology. Of course we are not aware of such precisely localized sources in our Universe, and the proposed effect remains well beyond the reach of current experiments. Our analysis, however, is useful to settle a question that was debated since the introduction of the $\kappa$-Poincaré group: does $\kappa$-Poincaré predict in vacuo dispersion of particles that can be detected with gamma ray burst observations? The commutator between quantum fields at spacelike separated points becomes zero already at a distance of a few units of $\sqrt{L \kappa^{-1}}$ from the classical light cone, which for $L \sim 1$ billion years and $\kappa^{-1} \sim L_{p}$ is $\sim 10$ fs. This is a time interval that is 14 orders of magnitude smaller than $1 \mathrm{~s}$. So at a distance of $1 \mathrm{sec}$ from the light cone there can be no signal transmission. We indeed have an effect of in vacuo dispersion of the kind that is considered by the literature on quantum-gravity phenomenology with gamma ray burst $[43,44]$, but the effect is enormously smaller than what would be required to be detected using gamma ray bursts.

Our conclusion is that gamma ray bursts are not capable of constraining models of quantum fields propagating on a $\kappa$-Poincaré background. We nonetheless want to stress that we find a distance-dependent effect. This means that for macroscopic distances the size of the nonlocal blurring is greatly boosted, which gives hope to detecting the effect by some other means. Contrast this result with previous claims [71], which attribute an invariably Planckian size to the nonlocal effect in $\kappa$-Minkowski, independently of the distances involved. Such effects would be in principle impossible to measure, and our results improve the situation dramatically for phenomenology (see also the comments in [70]).

The present work opens several lines of future investigation. QFT on a $\kappa$-Minkowski background should be further developed. We already remarked that the next natural step is to discuss discrete symmetries $(\mathrm{C}, \mathrm{P}$, and $\mathrm{T})$. Then an important issue is to understand conserved quantities, which provide the building blocks for asymptotic observables. The theory at that point would be developed enough to introduce interactions and develop dynamically nontrivial models. For what regards phenomenology, the two most urgent extensions of our work are on one hand the exploration of the dependence of the light cone on the choice of state of the quantum geometry. As we remarked in Sec. VA a feature of our effective theories can be deemed observable only if it is robust under change of the choice of state. On the other hand, the Pauli-Jordan function is suited to discuss causality issues (i.e., to tell whether two events can be causally connected, and, in our case, to exclude an apparently superluminal propagation of a certain magnitude). To make actual predictions regarding the waveform of signals propagating in a vacuum we need the two-point function. This can be calculated using the techniques developed in our paper, and will be the subject of future works. 


\section{ACKNOWLEDGMENTS}

F. M. has received funding from the European Union's research and innovation program under a Marie Skłodowska-Curie grant through the INdAM-COFUND2012 program of the Italian Institute of High Mathematics (INdAM).

\section{APPENDIX A: FINITE LORENTZ TRANSFORMATIONS IN MOMENTUM SPACE (IN 1 + 1 DIMENSIONS)}

In this Appendix, we want to derive Eq. (28), which we used first in Eq. (16). This is the expression for the finite Lorentz transformations on momenta that is implied by the (noncommutative) way in which $\kappa$-Poincaré coordinates transform under the left coaction of the $\kappa$-Poincare group:

$$
\begin{aligned}
\Delta_{L}\left[x^{\mu}\right] & =\Lambda_{\nu}^{\mu} \otimes x^{\nu}+a^{\mu} \otimes \mathbb{1} \Rightarrow \Delta_{L}\left[e^{i p_{i} x^{i}} e^{i p_{0} x^{0}}\right] \\
& =e^{i \lambda_{i}[p, \Lambda] \otimes x^{i}} e^{i \lambda_{0}[p, \Lambda] \otimes x^{0}} e^{i p_{i} a^{i} \otimes \mathbb{1}} e^{i p_{0} a^{0} \otimes \mathbb{1}} .
\end{aligned}
$$

In $1+1$ dimensions, we can write the $\kappa$-Poincaré group commutation relations (6) [in the timelike case (7)] as

$$
\begin{aligned}
{\left[a^{0}, a^{1}\right] } & =\frac{i}{\kappa} a^{1}, \quad\left[a^{0}, \xi\right]=\frac{i}{\kappa} \sinh \xi \\
{\left[a^{1}, \xi\right] } & =\frac{i}{\kappa}(\cosh \xi-1)
\end{aligned}
$$

where $\xi$ is the rapdity, $\cosh \xi \Lambda_{0}^{0}=\Lambda^{1}{ }_{1}, \sinh \xi \Lambda^{0}{ }_{1}=\Lambda^{1}{ }_{0}$. The commutation relations above can also be obtained from the request that the coaction $\Delta_{L}$ leaves the $\kappa$-Minkowski commutation relations invariant, i.e.,

$$
\begin{aligned}
& {\left[\cosh \xi x^{0}+\sinh \xi x^{1}+a^{0}, \cosh \xi x^{1}+\sinh \xi x^{0}+a^{1} \mathbb{1}\right]} \\
& \quad=\frac{i}{\kappa}\left(\cosh \xi x^{1}+\sinh \xi x^{0}+a^{1}\right),
\end{aligned}
$$

where, for simplicity, we omitted the tensor product $\otimes$ between group elements and coordinates, and we understand $x^{\mu}, \xi$, and $a^{\mu}$ as belonging to the same algebra, in which $x^{\mu}$ commute with $\xi$ and $a^{\mu}$ (this is just the standard construction of a tensor product algebra).

To find the explicit expression of $\lambda_{\mu}[p, \Lambda]$, we need to first calculate the adjoint action of $e^{i p_{0} a^{0}}$ and $e^{i p_{1} a^{1}}$ on $\xi$. To do so, we notice that the following nonlinear changes of variable

$$
\eta=\log \left(\tanh \frac{\xi}{2}\right), \quad \rho=-\operatorname{coth} \frac{\xi}{2}
$$

make the commutator with $a^{0}$, respectively, $a^{1}$, canonical:

$$
\left[a^{0}, \eta\right]=\frac{i}{\kappa}, \quad\left[a^{1}, \rho\right]=\frac{i}{\kappa} .
$$

Then the adjoint action of $e^{i p_{0} a^{0}}$ and $e^{i p_{1} a^{1}}$ on these algebra elements is a translation

$e^{i p_{0} a^{0}} \eta e^{-i p_{0} a^{0}}=\eta-\frac{p_{0}}{\kappa}, \quad e^{i p_{1} a^{1}} \rho e^{-i p_{1} a^{1}}=\rho-\frac{p_{1}}{\kappa}$

using the homomorphism property of the adjoint action and the inverses of the relations (A4):

$$
\begin{aligned}
& e^{i p_{0} a^{0}} \xi e^{-i p_{0} a^{0}}=2 \operatorname{arctanh}\left(e^{-\frac{p_{0}}{\kappa}} \tanh \frac{\xi}{2}\right), \\
& e^{i p_{1} a^{1}} \xi e^{-i p_{1} a^{1}}=2 \operatorname{arccoth}\left(\frac{p_{1}}{\kappa}+\operatorname{coth} \frac{\xi}{2}\right) .
\end{aligned}
$$

The homomorphism property implies that the above rules can be applied to an arbitrary function of $\xi$; in particular the following holds:

$$
\begin{aligned}
& e^{i p_{0} a^{0}} f(\xi)=f(\tilde{\xi}) e^{i p_{0} a^{0}}, \quad \tilde{\xi}=2 \operatorname{arctanh}\left(e^{-\frac{p_{0}}{\kappa}} \tanh \frac{\xi}{2}\right), \\
& e^{i p_{1} a^{1}} f(\xi)=f(\hat{\xi}) e^{i p_{1} a^{1}}, \quad \hat{\xi}=2 \operatorname{arccoth}\left(\frac{p_{1}}{\kappa}+\operatorname{coth} \frac{\xi}{2}\right) .
\end{aligned}
$$

Now we are interested in the adjoint action of (an arbitrary function of) $\xi$ on an ordered exponential of $a^{\mu}$. Consider the following induction chain:

$$
\begin{aligned}
e^{f(\xi)} a^{0}= & \left(a^{0}-\frac{i}{\kappa} \sinh \xi f^{\prime}(\xi)\right) e^{f(\xi)}, \\
e^{f(\xi)}\left(a^{0}\right)^{2}= & \left(a^{0}-\frac{i}{\kappa} \sinh \xi f^{\prime}(\xi)\right)^{2} e^{f(\xi)}, \\
& \vdots \\
e^{f(\xi)}\left(a^{0}\right)^{n}= & \left(a^{0}-\frac{i}{\kappa} \sinh \xi f^{\prime}(\xi)\right)^{n} e^{f(\xi)} .
\end{aligned}
$$

Therefore

$$
e^{f(\xi)} e^{i p_{0} a^{0}}=e^{i p_{0}\left(a^{0}-\frac{i}{\kappa} \sinh \xi f^{\prime}(\xi)\right)} e^{f(\xi)},
$$

which can be written as an ad-action of $f(\xi)$ on $e^{i p_{0} a^{0}}$ :

$$
e^{i p_{0} a^{0}+\frac{p_{0}}{\kappa} \sinh \xi f^{\prime}(\xi)}=e^{f(\xi)} e^{i p_{0} a^{0}} e^{-f(\xi)},
$$

and, similarly, in the case of $e^{i p_{1} a^{1}}$ :

$$
e^{i p_{1} a^{1}+\frac{p_{1}}{\kappa}(\cosh \xi-1) f^{\prime}(\xi)}=e^{f(\xi)} e^{i p_{1} a^{1}} e^{-f(\xi)} .
$$

The last two relations are true if $f(\xi)$ is a pure function of $\xi$. We are interested in the case in which it is a linear combination of $x^{0}$ and $x^{1}$ which, despite commuting 
with $\xi$, do not commute with each other. We can apply the rule unchanged when the exponent contains $x^{0}$ or $x^{1}$ alone (because they commute with $\xi$ and $a^{\mu}$, so it is convenient to consider the following ordered plane wave:

$$
e^{i k_{1}(\xi) x^{1}} e^{i k_{0}(\xi) x^{0}}
$$

where the coefficients $k_{\mu}(\xi)$ are arbitrary functions of $\xi$. Computing the commutator with $a^{0}$

$$
\begin{aligned}
e^{i k_{1}(\xi) x^{1}} e^{i k_{0}(\xi) x^{0}} a^{0} & =e^{i k_{1}(\xi) x^{1}}\left(a^{0}+\frac{1}{\kappa} \sinh \xi k_{0}{ }^{\prime}(\xi) x^{0}\right) e^{i k_{0}(\xi) x^{0}} \\
& =\left[a^{0}+\frac{1}{\kappa} \sinh \xi\left(k_{0}{ }^{\prime}(\xi) x^{0}+k_{1}{ }^{\prime}(\xi) x^{1}+\frac{1}{\kappa} k_{0}{ }^{\prime}(\xi) k_{1}(\xi) x^{1}\right)\right] e^{i k_{1}(\xi) x^{1}} e^{i k_{0}(\xi) x^{0}},
\end{aligned}
$$

where the last addend in the square brackets is a consequence of

$$
e^{i k_{1}(\xi) x^{1}} x^{0}=\left(x_{0}+\frac{1}{\kappa} k_{1}(\xi) x^{1}\right) e^{i k_{1}(\xi) x^{1}}
$$

which is easy proved using the commutation relations $\left[x^{0}, x^{1}\right]=\frac{i}{\kappa} x^{1}$. A similar relation is satisfied by $a^{1}$ :

$$
\begin{aligned}
e^{i k_{1}(\xi) x^{1}} e^{i k_{0}(\xi) x^{0}} a^{1} & =e^{i k_{1}(\xi) x^{1}}\left(a^{1}+\frac{1}{\kappa}(\cosh \xi-1) k_{0}{ }^{\prime}(\xi) x^{0}\right) e^{i k_{0}(\xi) x^{0}} \\
& =\left[a^{1}+\frac{1}{\kappa}(\cosh \xi-1)\left(k_{0}{ }^{\prime}(\xi) x^{0}+k_{1}{ }^{\prime}(\xi) x^{1}+\frac{1}{\kappa} k_{0}{ }^{\prime}(\xi) k_{1}(\xi) x^{1}\right)\right] e^{i k_{1}(\xi) x^{1}} e^{i k_{0}(\xi) x^{0}} .
\end{aligned}
$$

These relation hold too on powers of $a^{0}$ or $a^{1}$, and consequently also on exponentials:

$$
\begin{aligned}
& e^{i k_{1}(\xi) x^{1}} e^{i k_{0}(\xi) x^{0}} e^{i p_{1} a^{1}}=e^{i p_{1}\left[a^{1}+\frac{1}{\kappa}(\cosh \xi-1)\left(k_{0}{ }^{\prime}(\xi) x^{0}+k_{1}{ }^{\prime}(\xi) x^{1}+\frac{1}{\kappa} k_{0}{ }^{\prime}(\xi) k_{1}(\xi) x^{1}\right)\right]} e^{i k_{1}(\xi) x^{1}} e^{i k_{0}(\xi) x^{0}}, \\
& e^{i q_{1}(\xi) x^{1}} e^{i q_{0}(\xi) x^{0}} e^{i p_{0} a^{0}}=e^{i p_{0}\left[a^{0}+\frac{1}{\kappa} \sinh \xi\left(q_{0}{ }^{\prime}(\xi) x^{0}+q_{1}{ }^{\prime}(\xi) x^{1}+\frac{1}{\kappa} q_{0}{ }^{\prime}(\xi) q_{1}(\xi) x^{1}\right)\right]} e^{i q_{1}(\xi) x^{1}} e^{i q_{0}(\xi) x^{0}} .
\end{aligned}
$$

If we multiply both sides from the right by $e^{-i k_{0}(\xi) x^{0}} e^{-i k_{1}(\xi) x^{1}}$ and reorder the exponentials using (A8) we get two BCH formulas for our algebra:

$$
\begin{aligned}
& e^{i p_{1}\left[a^{1}+\frac{1}{\kappa}(\cosh \xi-1)\left(k_{0}{ }^{\prime} x^{0}+k_{1} x^{1}+\frac{1}{\kappa} k_{0}{ }^{\prime} k_{1} x^{1}\right)\right]}=e^{i k_{1} x^{1}} e^{i k_{0} x^{0}} e^{i p_{1} a^{1}} e^{-i k_{0} x^{0}} e^{-i k_{1} x^{1}} \\
& =e^{i k_{1} x^{1}} e^{i\left(k_{0}-\hat{k}_{0}\right) x^{0}} e^{-i \hat{k}_{1} x^{1}} e^{i p_{1} a^{1}} \\
& =e^{i\left(k_{1}-e^{-\left(k_{0}-\hat{k}_{0}\right) / \kappa} \hat{k}_{1}\right) x^{1}} e^{i\left(k_{0}-\hat{k}_{0}\right) x^{0}} e^{i p_{1} a^{1}}, \\
& e^{i p_{0}\left[a^{0}+\frac{1}{\kappa} \sinh \xi\left(q_{0}^{\prime} x^{0}+q_{1}{ }^{\prime} x^{1}+\frac{1}{\kappa} q_{0}{ }^{\prime} q_{1} x^{1}\right)\right]}=e^{i q_{1} x^{1}} e^{i q_{0} x^{0}} e^{i p_{0} a^{0}} e^{-i q_{0} x^{0}} e^{-i q_{1} x^{1}} \\
& =e^{i q_{1} x^{1}} e^{i\left(q_{0}-\tilde{q}_{0}\right) x^{0}} e^{-i \tilde{q}_{1} x^{1}} e^{i p_{0} a^{0}} \\
& =e^{i\left(q_{1}-e^{-\left(q_{0}-\tilde{q}_{0}\right) / \kappa} \tilde{q}_{1}\right) x^{1}} e^{i\left(q_{0}-\tilde{q}_{0}\right) x^{0}} e^{i p_{0} a^{0}},
\end{aligned}
$$

where we used the relation

$$
e^{a x^{0}} e^{b x^{1}}=e^{e^{i a / x} b x^{1}} e^{a x^{0}}
$$

and the notation $\hat{k}_{\mu}=k_{\mu}(\hat{\xi}), \tilde{k}_{\mu}=k_{\mu}(\tilde{\xi})$ is pretty self-explanatory. Multiplying the first equation to the second from the left:

$$
\begin{aligned}
& e^{i p_{1}\left[a^{1}+\frac{1}{\kappa}(\cosh \xi-1)\left(k_{0}{ }^{\prime} x^{0}+k_{1}{ }^{1} x^{1}+\frac{1}{\kappa} k_{0}{ }^{\prime} k_{1} x^{1}\right)\right]} e^{i p_{0}\left[a^{0}+\frac{1}{\kappa} \sinh \xi\left(q_{0}{ }^{\prime} x^{0}+q_{1}{ }^{1} x^{1}+\frac{1}{\kappa} q_{0}{ }^{\prime} q_{1} x^{1}\right)\right]} \\
& =e^{i\left(k_{1}-e^{-\left(k_{0}-\hat{k}_{0}\right) / \kappa} \hat{k}_{1}\right) x^{1}} e^{i\left(k_{0}-\hat{k}_{0}\right) x^{0}} e^{i p_{1} a^{1}} e^{i\left(q_{1}-e^{-\left(q_{0}-\tilde{q}_{0}\right) / \kappa} \tilde{q}_{1}\right) x^{1}} e^{i\left(q_{0}-\tilde{q}_{0}\right) x^{0}} e^{i p_{0} a^{0}} \\
& =e^{i\left(k_{1}-e^{-\left(k_{0}-\hat{k}_{0}\right) / \kappa} \hat{k}_{1}\right) x^{1}} e^{i\left(k_{0}-\hat{k}_{0}\right) x^{0}} e^{i\left(\hat{q}_{1}-e^{-\left(\hat{q}_{0}-\hat{q}_{0}\right) / \kappa} \hat{\tilde{q}}_{1}\right) x^{1}} e^{i\left(\hat{q}_{0}-\hat{\hat{q}}_{0}\right) x^{0}} e^{i p_{1} a^{1}} e^{i p_{0} a^{0}} \\
& =e^{i\left[k_{1}-e^{-\left(k_{0}-\hat{k}_{0}\right) / \kappa} \hat{k}_{1}+e^{-\left(k_{0}-\hat{k}_{0}\right) / \kappa}\left(\hat{q}_{1}-e^{-\left(\hat{q}_{0}-\hat{q}_{0}\right) / \kappa} \hat{\hat{q}}_{1}\right)\right] x^{1}} e^{i\left(k_{0}-\hat{k}_{0}+\hat{q}_{0}-\hat{q}_{0}\right) x^{0}} e^{i p_{1} a^{1}} e^{i p_{0} a^{0}} .
\end{aligned}
$$


The final step is to equate the left-hand ides of (A20) with

$$
\Delta_{L}\left[e^{i p_{1} x^{1}} e^{i p_{0} x^{1}}\right]=e^{i p_{1}\left(x^{1} \cosh \xi+x^{0} \sinh \xi\right)+i p_{1} a^{1}} e^{i p_{0}\left(x^{0} \cosh \xi+x^{1} \sinh \xi\right)+i p_{0} a^{0}} .
$$

This can be achieved by fixing the functions $k_{\mu}(\xi), q_{\mu}(\xi)$ through the following differential equations:

$$
\left\{\begin{array} { l } 
{ \frac { 1 } { \kappa } ( \operatorname { c o s h } \xi - 1 ) k _ { 0 } { } ^ { \prime } = \operatorname { s i n h } \xi , } \\
{ \frac { 1 } { \kappa } ( \operatorname { c o s h } \xi - 1 ) ( k _ { 1 } { } ^ { \prime } + \frac { 1 } { \kappa } k _ { 0 } { } ^ { \prime } k _ { 1 } ) = \operatorname { c o s h } \xi , }
\end{array} \quad \left\{\begin{array}{l}
\frac{1}{\kappa} \sinh \xi q_{0}{ }^{\prime}=\cosh \xi, \\
\frac{1}{\kappa} \sinh \xi\left(q_{1}{ }^{\prime}+\frac{1}{\kappa} q_{0}{ }^{\prime} q_{1}\right)=\sinh \xi .
\end{array}\right.\right.
$$

These are easily solved:

$$
\left\{\begin{array} { l } 
{ k _ { 0 } = \kappa [ 2 \operatorname { l o g } ( \operatorname { s i n h } \frac { \xi } { 2 } ) + c _ { 1 } ] , } \\
{ k _ { 1 } = \kappa [ \frac { \operatorname { s i n h } \xi + c _ { 2 } } { \operatorname { c o s h } \xi - 1 } ] }
\end{array} \quad \left\{\begin{array}{l}
q_{0}=\kappa\left[\log (\sinh \xi)+c_{3}\right] \\
q_{1}=\kappa\left[\frac{\cosh \xi+c_{4}}{\sinh \xi}\right]
\end{array}\right.\right.
$$

So the conclusion is the formula

$$
\Delta_{L}\left[e^{i p_{1} x^{1}} e^{i p_{0} x^{1}}\right]=e^{i \lambda_{1}[\xi, p] x^{1}} e^{i \lambda_{0}[\xi, p] x^{0}} e^{i p_{1} a^{1}} e^{i p_{0} a^{0}}
$$

where

$$
\left\{\begin{array}{l}
\lambda_{1}[\xi, p]=k_{1}-e^{-\left(k_{0}-\hat{k}_{0}\right) / \kappa} \hat{k}_{1}+e^{-\left(k_{0}-\hat{k}_{0}\right) / \kappa}\left(\hat{q}_{1}-e^{-\left(\hat{q}_{0}-\hat{\tilde{q}}_{0}\right) / \kappa} \hat{\tilde{q}}_{1}\right), \\
\lambda_{0}[\xi, p]=k_{0}-\hat{k}_{0}+\hat{q}_{0}-\hat{\tilde{q}}_{0},
\end{array}\right.
$$

in which

$$
\left\{\begin{array}{l}
\hat{k}_{\mu}=k_{\mu}[\hat{\xi}]=k_{\mu}\left[2 \operatorname{arccoth}\left(\frac{p_{1}}{\kappa}+\operatorname{coth} \frac{\xi}{2}\right)\right], \\
\tilde{k}_{\mu}=k_{\mu}[\tilde{\xi}]=k_{\mu}\left[2 \operatorname { a r c t a n h } \left(e^{\left.\left.-\frac{p_{0}}{\kappa} \tanh \frac{\xi}{2}\right)\right],}\right.\right. \\
\hat{\tilde{k}}_{\mu}=k_{\mu}\left[2 \operatorname{arctanh}\left(e^{-\frac{p_{0}}{\kappa}} \tanh \frac{\hat{\xi}}{2}\right)\right]=k_{\mu}\left[2 \operatorname{arctanh}\left(\frac{{\frac{p}{p_{1}}}^{-\frac{p_{0}}{\kappa}+\operatorname{coth} \frac{\xi}{2}}}{\xi}\right)\right]
\end{array}\right.
$$

and analogously for $q_{\mu}$. An explicit calculation reveals that

$$
\begin{aligned}
& \lambda_{0}[\xi, p]=p_{0}+\kappa \log \left[\left(\cosh \frac{\xi}{2}+\frac{p_{1}}{\kappa} \sinh \frac{\xi}{2}\right)^{2}-e^{-2 p_{0} / \kappa} \sinh ^{2} \frac{\xi}{2}\right], \\
& \lambda_{1}[\xi, p]=\kappa \frac{\left(\cosh \frac{\xi}{2}+\frac{p_{1}}{\kappa} \sinh \frac{\xi}{2}\right)\left(\sinh \frac{\xi}{2}+\frac{p_{1}}{\kappa} \cosh \frac{\xi}{2}\right)-e^{-2 p_{0} / \kappa} \cosh \frac{\xi}{2} \sinh \frac{\xi}{2}}{\left(\cosh \frac{\xi}{2}+\frac{p_{1}}{\kappa} \sinh \frac{\xi}{2}\right)^{2}-e^{-2 p_{0} / \kappa} \sinh ^{2} \frac{\xi}{2}}
\end{aligned}
$$

which is Eq. (28) which we used, initially, in Eq. (16).

The transformation rule above can be found by integrating the vector flow on momentum space that is generated by the action of infinitesimal Lorentz transformations [Eq. (19)] on the momenta. This was first done in [72]. Alternatively, one can use a matrix representation of the algebra (A2) and essentially do the same we did algebraically in this Appendix, as was done in [10].

\section{APPENDIX B: CALCULATION OF THE CREATION AND ANNIHILATION OPERATOR ALGEBRA}

It is convenient to introduce the following three new operators:

$$
\hat{d}(\mathbf{q})=e^{-\frac{3 \omega^{-}(\mathbf{q} \mid)}{\kappa}} \hat{a}^{\dagger}\left(\mathbf{S}_{-}(\mathbf{q})\right), \quad \hat{e}(\mathbf{q})=e^{-\frac{3 \omega^{+}(|\mathbf{q}|)}{\kappa}} \hat{b}\left(\mathbf{S}_{+}(\mathbf{q})\right), \quad \hat{f}(\mathbf{q})=e^{-\frac{3 \omega^{+}(\mathbf{q q} \mid)}{\kappa}} \hat{c}\left(\mathbf{S}_{+}(\mathbf{q})\right),
$$

which makes $\hat{\phi}^{\dagger}(x)$ in (63) take the same form as $\hat{\phi}(x)$ in Eq. (55). Expliciting the mode expansion of the fields in the commutation relations (80), 


$$
\begin{aligned}
{\left[\hat{\phi}(z), \hat{\phi}^{\dagger}(y)\right]=} & \int_{|\mathbf{p}|<\kappa} \frac{\mathrm{d}^{3} p e^{\frac{3 \omega^{+}(|\mathbf{p}|)}{\kappa}}}{2 \sqrt{m^{2}+|\mathbf{p}|^{2}}}\left(\int_{\mathbb{R}^{3}} \frac{\mathrm{d}^{3} q e^{\frac{3 \omega^{-}(|\mathbf{q}|)}{\kappa}}}{2 \sqrt{m^{2}+|\mathbf{q}|^{2}}}[\hat{a}(\mathbf{p}), \hat{d}(\mathbf{q})] e^{i \mathbf{p} \cdot \mathbf{z}+i \mathbf{q} \cdot \mathbf{y}} e^{i \omega^{+}(|\mathbf{p}|) z^{0}+i \omega^{-}(|\mathbf{q}|) y^{0}}\right. \\
& +\int_{|\mathbf{q}|<\kappa} \frac{\mathrm{d}^{3} q e^{\frac{3 \omega^{+}(|\mathbf{q}|)}{\kappa}}}{2 \sqrt{m^{2}+|\mathbf{q}|^{2}}}[\hat{a}(\mathbf{p}), \hat{e}(\mathbf{q})] e^{i \mathbf{p} \cdot \mathbf{z}+i \mathbf{q} \cdot \mathbf{y}} e^{i \omega^{+}(|\mathbf{p}|) z^{0}+i \omega^{+}(|\mathbf{q}|) y^{0}} \\
& \left.+\int_{|\mathbf{q}|>\kappa} \frac{\mathrm{d}^{3} q e^{\frac{3 \omega^{+}(|\mathbf{q}|)}{\kappa}}}{2 \sqrt{m^{2}+|\mathbf{q}|^{2}}}[\hat{a}(\mathbf{p}), \hat{f}(\mathbf{q})] e^{i \mathbf{p} \cdot \mathbf{z}+i \mathbf{q} \cdot \mathbf{y}} e^{i \omega^{+}(|\mathbf{p}|) z^{0}+i \omega^{+}(|\mathbf{q}|) y^{0}}\right) \\
& +\int_{\mathbb{R}^{3}} \frac{\mathrm{d}^{3} p e^{\frac{3 \omega^{-}(|\mathbf{p}|)}{\kappa}}}{2 \sqrt{m^{2}+|\mathbf{p}|^{2}}}\left(\int_{\mathbb{R}^{3}} \frac{\mathrm{d}^{3} q e^{\frac{3 \omega^{-}(|\mathbf{q}|)}{\kappa}}}{2 \sqrt{m^{2}+|\mathbf{q}|^{2}}}\left[\hat{b}^{\dagger}(\mathbf{p}), \hat{d}(\mathbf{q})\right] e^{i \mathbf{p} \cdot z+i \mathbf{q} \cdot \mathbf{y}} e^{i \omega^{-}(|\mathbf{p}|) z^{0}+i \omega^{-}(|\mathbf{q}|) y^{0}}\right. \\
& +\int_{|\mathbf{q}|<\kappa} \frac{\mathrm{d}^{3} q e^{\frac{3 \omega^{+}(|\mathbf{q}|)}{\kappa}}}{2 \sqrt{m^{2}+|\mathbf{q}|^{2}}}\left[\hat{b}^{\dagger}(\mathbf{p}), \hat{e}(\mathbf{q})\right] e^{i \mathbf{p} \cdot \mathbf{z}+i \mathbf{q} \cdot \mathbf{y}} e^{i \omega^{-}(|\mathbf{p}|) z^{0}+i \omega^{+}(|\mathbf{q}|) y^{0}} \\
& \left.+\int_{|\mathbf{q}|>\kappa} \frac{\mathrm{d}^{3} q e^{\frac{3 \omega^{+}(|\mathbf{q}|)}{\kappa}}}{2 \sqrt{m^{2}+|\mathbf{q}|^{2}}}\left[\hat{b}^{\dagger}(\mathbf{p}), \hat{f}(\mathbf{q})\right] e^{i \mathbf{p} \cdot \mathbf{z}+i \mathbf{q} \cdot \mathbf{y}} e^{i \omega^{-}(|\mathbf{p}|) z^{0}+i \omega^{+}(|\mathbf{q}|) y^{0}}\right) \\
& +\int_{|\mathbf{p}|>\kappa} \frac{\mathrm{d}^{3} p e^{\frac{3 \omega^{+}(|\mathbf{p}|)}{\kappa}}}{2 \sqrt{m^{2}+|\mathbf{p}|^{2}}}\left(\int_{\mathbb{R}^{3}} \frac{\mathrm{d}^{3} q e^{\frac{3 \omega^{-}(|\mathbf{q}|)}{\kappa}}}{2 \sqrt{m^{2}+|\mathbf{q}|^{2}}}\left[\hat{c}^{\dagger}(\mathbf{p}), \hat{d}(\mathbf{q})\right] e^{i \mathbf{p} \cdot \mathbf{z}+i \mathbf{q} \cdot \mathbf{y}} e^{i \omega^{+}(|\mathbf{p}|) z^{0}+i \omega^{-}(|\mathbf{q}|) y^{0}}\right. \\
& +\int_{|\mathbf{q}|<\kappa} \frac{\mathrm{d}^{3} q e^{\frac{3 \omega^{+}(|\mathbf{q}|)}{\kappa}}}{2 \sqrt{m^{2}+|\mathbf{q}|^{2}}}\left[\hat{c}^{\dagger}(\mathbf{p}), \hat{e}(\mathbf{q})\right] e^{i \mathbf{p} \cdot \mathbf{z}+i \mathbf{q} \cdot \mathbf{y}} e^{i \omega^{+}(|\mathbf{p}|) z^{0}+i \omega^{+}(|\mathbf{q}|) y^{0}} \\
& \left.+\int_{|\mathbf{q}|>\kappa} \frac{\mathrm{d}^{3} q e^{\frac{3 \omega^{+}(|\mathbf{q}|)}{\kappa}}}{2 \sqrt{m^{2}+|\mathbf{q}|^{2}}}\left[\hat{c}^{\dagger}(\mathbf{p}), \hat{f}(\mathbf{q})\right] e^{i \mathbf{p} \cdot \mathbf{z}+i \mathbf{q} \cdot \mathbf{y}} e^{i \omega^{+}(|\mathbf{p}|) z^{0}+i \omega^{+}(|\mathbf{q}|) y^{0}}\right) .
\end{aligned}
$$

Comparing with (76) reveals that the three terms above should be identified pairwise with the three terms in the Pauli-Jordan function:

$$
\begin{aligned}
& \left(\int_{\mathbb{R}^{3}} \frac{\mathrm{d}^{3} q e^{\frac{3 \omega^{-}(\mathbf{q})}{\kappa}}}{2 \sqrt{m^{2}+|\mathbf{q}|^{2}}}[\hat{a}(\mathbf{p}), \hat{d}(\mathbf{q})] e^{i \mathbf{p} \cdot \mathbf{z}+i \mathbf{q} \cdot \mathbf{y}} e^{i \omega^{+}(\mathbf{p}) z^{0}+i \omega^{-}(\mathbf{q}) y^{0}}\right. \\
& \quad+\int_{|\mathbf{q}|<\kappa} \frac{\mathrm{d}^{3} q e^{\frac{3 \omega^{+}(\mathbf{q})}{\kappa}}}{2 \sqrt{m^{2}+|\mathbf{q}|^{2}}}[\hat{a}(\mathbf{p}), \hat{e}(\mathbf{q})] e^{i \mathbf{p} \cdot \mathbf{z}+i \mathbf{q} \cdot \mathbf{y}} e^{i \omega \omega^{+}(\mathbf{p}) z^{0}+i \omega^{+}(\mathbf{q}) y^{0}} \\
& \left.\quad+\int_{|\mathbf{q}|>\kappa} \frac{\mathrm{d}^{3} q e^{\frac{3 \omega^{+}(\mathbf{q})}{\kappa}}}{2 \sqrt{m^{2}+|\mathbf{q}|^{2}}}[\hat{a}(\mathbf{p}), \hat{f}(\mathbf{q})] e^{i \mathbf{p} \cdot \mathbf{z}+i \mathbf{q} \cdot \mathbf{y}} e^{i \omega^{+}(\mathbf{p}) z^{0}+i \omega^{+}(\mathbf{q}) y^{0}}\right)=e^{i \mathbf{p} \cdot \mathbf{z}-i e^{\frac{\omega^{+}(\mathbf{p})}{\kappa}} \mathbf{p} \cdot \mathbf{y}} e^{i \omega^{+}(\mathbf{p})\left(z^{0}-y^{0}\right)} \\
& \left(\int_{\mathbb{R}^{3}} \frac{\mathrm{d}^{3} q e^{\frac{3 \omega^{-}(\mathbf{q})}{\kappa}}}{2 \sqrt{m^{2}+|\mathbf{q}|^{2}}}\left[\hat{b}^{\dagger}(\mathbf{p}), \hat{d}(\mathbf{q})\right] e^{i \mathbf{p} \cdot \mathbf{z}+i \mathbf{q} \cdot \mathbf{y}} e^{i \omega^{-}(\mathbf{p}) z^{0}+i \omega^{-}(\mathbf{q}) y^{0}}\right. \\
& \quad+\int_{|\mathbf{q}|<\kappa} \frac{\mathrm{d}^{3} q e^{\frac{3 \omega^{+}(\mathbf{q})}{\kappa}}}{2 \sqrt{m^{2}+|\mathbf{q}|^{2}}}\left[\hat{b}^{\dagger}(\mathbf{p}), \hat{e}(\mathbf{q})\right] e^{i \mathbf{p} \cdot \mathbf{z}+i \mathbf{q} \cdot \mathbf{y}} e^{i \omega^{-}(\mathbf{p}) z^{0}+i \omega^{+}(\mathbf{q}) y^{0}} \\
& \left.\quad+\int_{|\mathbf{q}|>\kappa} \frac{\mathrm{d}^{3} q e^{\frac{3 \omega^{+}(\mathbf{q})}{\kappa}}}{2 \sqrt{m^{2}+|\mathbf{q}|^{2}}}\left[\hat{b}^{\dagger}(\mathbf{p}), \hat{f}(\mathbf{q})\right] e^{i \mathbf{p} \cdot \mathbf{z}+i \mathbf{q} \cdot \mathbf{y}} e^{i \omega^{-}(\mathbf{p}) z^{0}+i \omega^{+}(\mathbf{q}) y^{0}}\right)=-e^{i \mathbf{p} \cdot \mathbf{z}} e^{-i e e^{\frac{\omega^{-}(\mathbf{p})}{\kappa}} \mathbf{p} \cdot \mathbf{y}} e^{i \omega^{-}(\mathbf{p})\left(z^{0}-y^{0}\right)}
\end{aligned}
$$




$$
\begin{aligned}
& \left(\int_{\mathbb{R}^{3}} \frac{\mathrm{d}^{3} q e^{\frac{3 \omega^{-}(\mathbf{q})}{\kappa}}}{2 \sqrt{m^{2}+|\mathbf{q}|^{2}}}\left[\hat{c}^{\dagger}(\mathbf{p}), \hat{d}(\mathbf{q})\right] e^{i \mathbf{p} \cdot \mathbf{z}+i \mathbf{q} \cdot \mathbf{y}} e^{i \omega^{+}(\mathbf{p}) z^{0}+i \omega^{-}(\mathbf{q}) y^{0}}\right. \\
& \quad+\int_{|\mathbf{q}|<\kappa} \frac{\mathrm{d}^{3} q e^{\frac{3 \omega^{+}(\mathbf{q})}{\kappa}}}{2 \sqrt{m^{2}+|\mathbf{q}|^{2}}}\left[\hat{c}^{\dagger}(\mathbf{p}), \hat{e}(\mathbf{q})\right] e^{i \mathbf{p} \cdot \mathbf{z}+i \mathbf{q} \cdot \mathbf{y}} e^{i \omega^{+}(\mathbf{p}) z^{0}+i \omega^{+}(\mathbf{q}) y^{0}} \\
& \left.\quad+\int_{|\mathbf{q}|>\kappa} \frac{\mathrm{d}^{3} q e^{\frac{3 \omega^{+}(\mathbf{q})}{\kappa}}}{2 \sqrt{m^{2}+|\mathbf{q}|^{2}}}\left[\hat{c}^{\dagger}(\mathbf{p}), \hat{f}(\mathbf{q})\right] e^{i \mathbf{p} \cdot \mathbf{z}+i \mathbf{q} \cdot \mathbf{y}} e^{i \omega^{+}(\mathbf{p}) z^{0}+i \omega^{+}(\mathbf{q}) y^{0}}\right)=-e^{i \mathbf{p} \cdot \mathbf{z}} e^{-i e^{\frac{\omega^{+}(\mathbf{p})}{\kappa}} \mathbf{p} \cdot \mathbf{y}} e^{i \omega^{+}(\mathbf{p})\left(z^{0}-y^{0}\right)} .
\end{aligned}
$$

Let us introduce the following ansatz:

$$
\begin{aligned}
{[\hat{a}(\mathbf{p}), \hat{d}(\mathbf{q})] } & =F_{a a} \delta^{(3)}\left[\mathbf{q}-\mathbf{S}_{+}(\mathbf{p})\right], & & {[\hat{a}(\mathbf{p}), \hat{e}(\mathbf{q})]=F_{a b} \delta^{(3)}\left[\mathbf{q}-\mathbf{S}_{+}(\mathbf{p})\right], } & & {[\hat{a}(\mathbf{p}), \hat{f}(\mathbf{q})]=F_{a c} \delta^{(3)}\left[\mathbf{q}-\mathbf{S}_{+}(\mathbf{p})\right], } \\
{\left[\hat{b}^{\dagger}(\mathbf{p}), \hat{d}(\mathbf{q})\right] } & =F_{b a} \delta^{(3)}\left[\mathbf{q}-\mathbf{S}_{-}(\mathbf{p})\right], & & {\left[\hat{b}^{\dagger}(\mathbf{p}), \hat{e}(\mathbf{q})\right]=F_{b b} \delta^{(3)}\left[\mathbf{q}-\mathbf{S}_{-}(\mathbf{p})\right], } & & {\left[\hat{b}^{\dagger}(\mathbf{p}), \hat{f}(\mathbf{q})\right]=F_{b c} \delta^{(3)}\left[\mathbf{q}-\mathbf{S}_{-}(\mathbf{p})\right], } \\
{\left[\hat{c}^{\dagger}(\mathbf{p}), \hat{d}(\mathbf{q})\right] } & =F_{c a} \delta^{(3)}\left[\mathbf{q}-\mathbf{S}_{+}(\mathbf{p})\right], & & {\left[\hat{c}^{\dagger}(\mathbf{p}), \hat{e}(\mathbf{q})\right]=F_{c b} \delta^{(3)}\left[\mathbf{q}-\mathbf{S}_{+}(\mathbf{p})\right], } & & {\left[\hat{c}^{\dagger}(\mathbf{p}), \hat{f}(\mathbf{q})\right]=F_{c c} \delta^{(3)}\left[\mathbf{q}-\mathbf{S}_{+}(\mathbf{p})\right] . }
\end{aligned}
$$

The first equation, Eq. (B3), turns into:

$$
\begin{aligned}
& \frac{e^{i \mathbf{p} \cdot \mathbf{z}+i \mathbf{S}_{+}(\mathbf{p}) \cdot \mathbf{y}} e^{i \omega^{+}(\mathbf{p}) z^{0}}}{2 \sqrt{m^{2}+\left|\mathbf{S}_{+}(\mathbf{p})\right|^{2}}}\left(e^{\frac{3 \omega^{-}(\mathbf{S}+(\mathbf{p}))}{\kappa}} F_{a a} e^{i \omega^{-}\left(\mathbf{S}_{+}(\mathbf{p})\right) y^{0}}+\Theta\left(\left|\mathbf{S}_{+}(\mathbf{p})\right|<\kappa\right) e^{\frac{3 \omega^{+}\left(\mathbf{S}_{+}(\mathbf{p})\right)}{\kappa}} F_{a b} e^{i \omega^{+}\left(\mathbf{S}_{+}(\mathbf{p})\right) y^{0}}\right. \\
& \left.\quad+\Theta\left(\left|\mathbf{S}_{+}(\mathbf{p})\right|>\kappa\right) e^{\frac{3 \omega^{+}\left(\mathbf{S}_{+}(\mathbf{p})\right)}{\kappa}} F_{a c} e^{i \omega^{+}\left(\mathbf{S}_{+}(\mathbf{p})\right) y^{0}}\right)\left.\right|_{|\mathbf{p}|<\kappa}=e^{i \mathbf{p} \cdot \mathbf{z}-i e^{\frac{\omega^{+}(\mathbf{p})}{\kappa}} \mathbf{p} \cdot \mathbf{y}} e^{i \omega^{+}(\mathbf{p})\left(z^{0}-y^{0}\right)}
\end{aligned}
$$

and using the relations (61), in particular $\omega^{-}\left(\mathbf{S}_{+}(\mathbf{p})\right)=-\omega^{+}(\mathbf{p})$ if $|\mathbf{p}|<\kappa$, we see that

$$
\left.e^{i \omega^{-}\left(\mathbf{S}_{+}(\mathbf{p})\right) y^{0}}\right|_{|\mathbf{p}|<\kappa}=e^{-i \omega^{+}(\mathbf{p}) y^{0}},\left.\quad e^{i \omega^{+}\left(\mathbf{S}_{+}(\mathbf{p})\right) y^{0}}\right|_{|\mathbf{p}|<\kappa} \neq e^{-i \omega^{+}(\mathbf{p}) y^{0}},
$$

and so only the term multiplying $F_{a a}$ is identical to the right-hand side. The second equation, Eq. (B4), is

$$
\begin{aligned}
& \frac{e^{i \mathbf{p} \cdot \mathbf{z}+i \mathbf{S}_{-}(\mathbf{p}) \cdot \mathbf{y}} e^{i \omega^{-}(\mathbf{p}) z^{0}}}{2 \sqrt{m^{2}+\left|\mathbf{S}_{-}(\mathbf{p})\right|^{2}}}\left(e^{\frac{3 \omega^{-}\left(\mathbf{S}_{-}(\mathbf{p})\right)}{\kappa}} F_{b a} e^{i \omega^{-}\left(\mathbf{S}_{-}(\mathbf{p})\right) y^{0}}+\Theta\left(\left|\mathbf{S}_{-}(\mathbf{p})\right|<\kappa\right) e^{\frac{3 \omega^{+}\left(\mathbf{S}_{-}(\mathbf{p})\right)}{\kappa}} F_{b b} e^{i \omega^{+}\left(\mathbf{S}_{-}(\mathbf{p})\right) y^{0}}\right. \\
& \left.+\Theta\left(\left|\mathbf{S}_{-}(\mathbf{p})\right|>\kappa\right) e^{\frac{3 \omega^{+}\left(\mathbf{S}_{-}(\mathbf{p})\right)}{\kappa}} F_{b c} e^{i \omega^{+}\left(\mathbf{S}_{-}(\mathbf{p})\right) y^{0}}\right)\left.\right|_{\mathbf{p} \in \mathbb{R}^{3}}=e^{i \mathbf{p} \cdot \mathbf{z}-i e^{\frac{\omega^{-}(\mathbf{p})}{\kappa}} \mathbf{p} \cdot \mathbf{y}} e^{i \omega^{-}(\mathbf{p})\left(z^{0}-y^{0}\right)},
\end{aligned}
$$

where the third term is crossed because $\left.\Theta\left(\left|\mathbf{S}_{-}(\mathbf{p})\right|>\kappa\right)\right|_{\mathbf{p} \in \mathbb{R}^{3}}=0$. Another look at relations (61), in particular $\omega^{+}\left(\mathbf{S}_{-}(\mathbf{p})\right)=-\omega^{-}(\mathbf{p})$ if $\mathbf{p} \in \mathbb{R}^{3}$, reveals that:

$$
\left.e^{i \omega^{-}\left(\mathbf{S}_{-}(\mathbf{p})\right) y^{0}}\right|_{\mathbf{p} \in \mathbb{R}^{3}} \neq e^{-i \omega^{-}(\mathbf{p}) y^{0}},\left.\quad e^{i \omega^{+}\left(\mathbf{S}_{-}(\mathbf{p})\right) y^{0}}\right|_{\mathbf{p} \in \mathbb{R}^{3}}=e^{-i \omega^{-}(\mathbf{p}) y^{0}},
$$

so only the $F_{b b}$ term survives. Finally consider Eq. (B5):

$$
\begin{aligned}
& \frac{e^{i \mathbf{p} \cdot \mathbf{z}+i \mathbf{S}_{+}(\mathbf{p}) \cdot \mathbf{y}} e^{i \omega^{+}(\mathbf{p}) z^{0}}}{2 \sqrt{m^{2}+\left|\mathbf{S}_{+}(\mathbf{p})\right|^{2}}}\left(e^{\frac{3 \omega^{-}\left(\mathbf{S}_{+}(\mathbf{p})\right)}{\kappa}} F_{c a} e^{i \omega^{-}\left(\mathbf{S}_{+}(\mathbf{p})\right) y^{0}}+\Theta\left(\left|\mathbf{S}_{+}(\mathbf{p})\right|<\kappa\right) e^{\frac{3 \omega^{+}\left(\mathbf{S}_{+}(\mathbf{p})\right)}{\kappa}} F_{c b} e^{i \omega^{+}\left(\mathbf{S}_{+}(\mathbf{p})\right) y^{0}}\right. \\
& \left.+\Theta\left(\left|\mathbf{S}_{+}(\mathbf{p})\right|>\kappa\right) e^{\frac{3 \omega^{+}\left(\mathbf{S}_{+}(\mathbf{p})\right)}{\kappa}} F_{c c} e^{i \omega^{+}\left(\mathbf{S}_{+}(\mathbf{p})\right) y^{0}}\right)\left.\right|_{|\mathbf{p}|>\kappa}=e^{i \mathbf{p} \cdot \mathbf{z}-i e^{\frac{\omega^{+}(\mathbf{p})}{\kappa}} \mathbf{p} \cdot \mathbf{y}} e^{i \omega^{+}(\mathbf{p})\left(z^{0}-y^{0}\right)},
\end{aligned}
$$

with relations (61), in particular $\omega^{+}\left(\mathbf{S}_{+}(\mathbf{p})\right)=-\omega^{+}(\mathbf{p})$ if $|\mathbf{p}|>\kappa$ :

$$
\left.e^{i \omega^{-}\left(\mathbf{S}_{+}(\mathbf{p})\right) y^{0}}\right|_{|\mathbf{p}|>\kappa} \neq e^{-i \omega^{+}(\mathbf{p}) y^{0}},\left.\quad e^{i \omega^{+}\left(\mathbf{S}_{+}(\mathbf{p})\right) y^{0}}\right|_{|\mathbf{p}|>\kappa}=e^{-i \omega^{+}(\mathbf{p}) y^{0}} .
$$

We see that only the $F_{c c}$ term survives. The three surviving coefficients then are constrained to be 


$$
F_{a a}=2 \sqrt{m^{2}+\left|\mathbf{S}_{+}(\mathbf{p})\right|^{2}} e^{\frac{3 \omega^{+}(\mathbf{p})}{\kappa}}, \quad F_{b b}=-2 \sqrt{m^{2}+\left|\mathbf{S}_{-}(\mathbf{p})\right|^{2}} e^{\frac{3 \omega^{-}(\mathbf{p})}{k}}, \quad F_{c c}=-2 \sqrt{m^{2}+\left|\mathbf{S}_{+}(\mathbf{p})\right|^{2}} e^{\frac{3 \omega^{+}(\mathbf{p})}{\kappa}},
$$

and the commutation relations are therefore,

$$
\begin{array}{r}
{[\hat{a}(\mathbf{p}), \hat{d}(\mathbf{q})]=2 \sqrt{m^{2}+\left|\mathbf{S}_{+}(\mathbf{p})\right|^{2}} e^{\frac{3 \omega^{+}(\mathbf{p})}{\kappa}} \delta^{(3)}\left[\mathbf{q}-\mathbf{S}_{+}(\mathbf{p})\right],} \\
{\left[\hat{b}^{\dagger}(\mathbf{p}), \hat{e}(\mathbf{q})\right]=-2 \sqrt{m^{2}+\left|\mathbf{S}_{-}(\mathbf{p})\right|^{2}} e^{\frac{3 \omega^{-}(\mathbf{p})}{\kappa}} \delta^{(3)}\left[\mathbf{q}-\mathbf{S}_{-}(\mathbf{p})\right],} \\
{\left[\hat{c}^{\dagger}(\mathbf{p}), \hat{f}(\mathbf{q})\right]=-2 \sqrt{m^{2}+\left|\mathbf{S}_{+}(\mathbf{p})\right|^{2}} e^{\frac{3 \omega^{+}(\mathbf{p})}{\kappa}} \delta^{(3)}\left[\mathbf{q}-\mathbf{S}_{+}(\mathbf{p})\right] .}
\end{array}
$$

We can change variables in the delta functions on the right-hand sides, and isolate $\mathbf{p}$. Then the delta functions produce the determinant of a Jacobian that cancels with the exponential terms multiplying them:

$$
\begin{array}{r}
\text { if }|\mathbf{p}|<\kappa \quad e^{\frac{3 \omega^{+}(\mathbf{p})}{\kappa}} \delta^{(3)}\left[\mathbf{q}-\mathbf{S}_{+}(\mathbf{p})\right]=\frac{e^{\frac{3 \omega^{+}(\mathbf{p})}{\kappa}}}{\left|\operatorname{det} \frac{\partial \mathbf{S}_{+}(\mathbf{p})}{\partial \mathbf{p}}\right|} \delta^{(3)}\left[\mathbf{p}-\mathbf{S}_{-}(\mathbf{q})\right]=\delta^{(3)}\left[\mathbf{p}-\mathbf{S}_{-}(\mathbf{q})\right], \\
\forall \mathbf{p} \quad e^{\frac{3 \omega^{-(\mathbf{p})}}{\kappa}} \delta^{(3)}\left[\mathbf{q}-\mathbf{S}_{-}(\mathbf{p})\right]=\frac{e^{\frac{3 \omega^{-}(\mathbf{p})}{\kappa}}}{\left|\operatorname{det} \frac{\partial \mathbf{S}_{-}(\mathbf{p})}{\partial \mathbf{p}}\right|} \delta^{(3)}\left[\mathbf{p}-\mathbf{S}_{+}(\mathbf{q})\right]=\delta^{(3)}\left[\mathbf{p}-\mathbf{S}_{+}(\mathbf{q})\right], \\
\text { if }|\mathbf{p}|>\kappa \quad e^{\frac{3 \omega^{+}(\mathbf{p})}{\kappa}} \delta^{(3)}\left[\mathbf{q}-\mathbf{S}_{+}(\mathbf{p})\right]=\frac{e^{\frac{3 \omega^{+}(\mathbf{p})}{\kappa}}}{\left|\operatorname{det} \frac{\partial \mathbf{S}_{+}(\mathbf{p})}{\partial \mathbf{p}}\right|} \delta^{(3)}\left[\mathbf{p}-\mathbf{S}_{+}(\mathbf{q})\right]=\delta^{(3)}\left[\mathbf{p}-\mathbf{S}_{+}(\mathbf{q})\right],
\end{array}
$$

then the commutation relations take a particularly simple form:

$$
\begin{gathered}
{[\hat{a}(\mathbf{p}), \hat{d}(\mathbf{q})]=2 \sqrt{m^{2}+|\mathbf{q}|^{2}} \delta^{(3)}\left[\mathbf{p}-\mathbf{S}_{-}(\mathbf{q})\right],} \\
{\left[\hat{b}^{\dagger}(\mathbf{p}), \hat{e}(\mathbf{q})\right]=-2 \sqrt{m^{2}+|\mathbf{q}|^{2}} \delta^{(3)}\left[\mathbf{p}-\mathbf{S}_{+}(\mathbf{q})\right],} \\
{\left[\hat{c}^{\dagger}(\mathbf{p}), \hat{f}(\mathbf{q})\right]=-2 \sqrt{m^{2}+|\mathbf{q}|^{2}} \delta^{(3)}\left[\mathbf{p}-\mathbf{S}_{+}(\mathbf{q})\right] .}
\end{gathered}
$$

Now use the definition (B1)

$$
\begin{aligned}
& {\left[\hat{a}(\mathbf{p}), \hat{a}^{\dagger}\left(\mathbf{S}_{-}(\mathbf{q})\right)\right]=2 e^{\frac{3 \omega^{-(|q| q)}}{\kappa}} \sqrt{m^{2}+|\mathbf{q}|^{2}} \delta^{(3)}\left[\mathbf{p}-\mathbf{S}_{-}(\mathbf{q})\right],} \\
& {\left[\hat{b}^{\dagger}(\mathbf{p}), \hat{b}\left(\mathbf{S}_{+}(\mathbf{q})\right)\right]=-2 e^{\frac{3 \omega^{+}(|\mathbf{q}|)}{\kappa}} \sqrt{m^{2}+|\mathbf{q}|^{2}} \delta^{(3)}\left[\mathbf{p}-\mathbf{S}_{+}(\mathbf{q})\right],} \\
& {\left[\hat{c}^{\dagger}(\mathbf{p}), \hat{c}\left(\mathbf{S}_{+}(\mathbf{q})\right)\right]=-2 e^{\frac{3 \omega^{+}(|\mathbf{q}|)}{\kappa}} \sqrt{m^{2}+|\mathbf{q}|^{2}} \delta^{(3)}\left[\mathbf{p}-\mathbf{S}_{+}(\mathbf{q})\right],}
\end{aligned}
$$

and recall Eq. (59):

$$
\begin{array}{cl}
\mathbf{S}_{+}\left(\mathbf{S}_{+}(\mathbf{p})\right)=\mathbf{p} & \text { if }|\mathbf{p}|>\kappa, \quad \mathbf{S}_{-}\left(\mathbf{S}_{+}(\mathbf{p})\right)=\mathbf{p} \\
\text { if }|\mathbf{p}|<\kappa, & \mathbf{S}_{+}\left(\mathbf{S}_{-}(\mathbf{p})\right)=\mathbf{p} \quad \forall \mathbf{p} \in \mathbb{R}^{3},
\end{array}
$$

and we get $\left[\hat{a}(\mathbf{p}), \hat{a}^{\dagger}(\mathbf{k})\right]=2 e^{\frac{3 \omega^{-}\left(\mathbf{S}_{+}(\mathbf{k})\right)}{\kappa}} \sqrt{m^{2}+\left|\mathbf{S}_{+}(\mathbf{k})\right|^{2}} \delta^{(3)}[\mathbf{p}-\mathbf{k}]$,

$$
\begin{aligned}
& {\left[\hat{b}^{\dagger}(\mathbf{p}), \hat{b}(\mathbf{k})\right]=-2 e^{\frac{3 \omega^{+}\left(\mathbf{S}_{-}(\mathbf{k})\right)}{\kappa}} \sqrt{m^{2}+\left|\mathbf{S}_{-}(\mathbf{k})\right|^{2}} \delta^{(3)}[\mathbf{p}-\mathbf{k}],} \\
& {\left[\hat{c}^{\dagger}(\mathbf{p}), \hat{c}(\mathbf{k})\right]=-2 e^{\frac{3 \omega^{+}\left(\mathbf{S}_{+}(\mathbf{k})\right)}{k}} \sqrt{m^{2}+\left|\mathbf{S}_{+}(\mathbf{k})\right|^{2}} \delta^{(3)}[\mathbf{p}-\mathbf{k}] .}
\end{aligned}
$$

Finally, using one last time Eq. (61):

$$
\begin{array}{ll}
\omega^{+}\left(\mathbf{S}_{-}(\mathbf{q})\right)=-\omega^{-}(\mathbf{q}), & \text { if } \mathbf{q} \in \mathbb{R}^{3}, \\
\omega^{-}\left(\mathbf{S}_{+}(\mathbf{q})\right)=-\omega^{+}(\mathbf{q}), & \text { if }|\mathbf{q}|<\kappa, \\
\omega^{+}\left(\mathbf{S}_{+}(\mathbf{q})\right)=-\omega^{+}(\mathbf{q}), & \text { if }|\mathbf{q}|>\kappa,
\end{array}
$$

we get the final form of the commutation relations:

$$
\begin{aligned}
& {\left[\hat{a}(\mathbf{p}), \hat{a}^{\dagger}(\mathbf{k})\right]=2 e^{-\frac{3 \omega^{+}(\mathbf{k})}{\kappa}} \sqrt{m^{2}+\left|\mathbf{S}_{+}(\mathbf{k})\right|^{2}} \delta^{(3)}[\mathbf{p}-\mathbf{k}],} \\
& {\left[\hat{b}^{\dagger}(\mathbf{p}), \hat{b}(\mathbf{k})\right]=-2 e^{-\frac{3 \omega^{-}(\mathbf{k})}{\kappa}} \sqrt{m^{2}+\left|\mathbf{S}_{-}(\mathbf{k})\right|^{2}} \delta^{(3)}[\mathbf{p}-\mathbf{k}],} \\
& {\left[\hat{c}^{\dagger}(\mathbf{p}), \hat{c}(\mathbf{k})\right]=-2 e^{-\frac{3 \omega^{+}(\mathbf{k})}{\kappa}} \sqrt{m^{2}+\left|\mathbf{S}_{+}(\mathbf{k})\right|^{2}} \delta^{(3)}[\mathbf{p}-\mathbf{k}] .}
\end{aligned}
$$


[1] H.-J. Matschull and M. Welling, Quantum mechanics of a point particle in $(2+1)$-dimensional gravity, Classical Quantum Gravity 15, 2981 (1998).

[2] L. Freidel and E. R. Livine, 3D Quantum Gravity and Effective Noncommutative Quantum Field Theory, Phys. Rev. Lett. 96, 221301 (2006).

[3] F. Lizzi, The structure of spacetime and noncommutative geometry, arXiv:0811.0268.

[4] P. Aschieri, M. Dimitrijevic, P. Kulish, F. Lizzi, and J. Wess, Noncommutative spacetimes: Symmetries in noncommutative geometry and field theory, Lecture Notes in Physics (Springer-Verlag, Berlin, Heidelberg, 2009).

[5] S. L. Woronowicz, Compact matrix pseudogroups, Commun. Math. Phys. 111, 613 (1987).

[6] S. Majid, A Quantum Groups Primer, London Mathematical Society Lecture Note Series (Cambridge University Press, Cambridge, England, 2002), Vol. 292.

[7] S. Zakrzewski, Poisson structures on the Poincaré group, Commun. Math. Phys. 185, 285 (1997).

[8] F. Mercati and M. Sergola, Physical constraints on quantum deformations of spacetime symmetries, Nucl. Phys. B933, 320 (2018).

[9] J. Lukierski and H. Ruegg, Quantum $\kappa$-Poincaré in any dimension, Phys. Lett. B 329, 189 (1994).

[10] S. Majid, Algebraic approach to quantum gravity II: noncommutative spacetime, arXiv:hep-th/0604130.

[11] P. Kosiński, J. Lukierski, and P. Maślanka, Local $D=4$ field theory on $\kappa$-deformed Minkowski space, Phys. Rev. D 62, 025004 (2000).

[12] P. Kosinski, J. Lukierski, and P. Maslanka, $\kappa$ deformed Wigner construction of relativistic wave functions and free fields on $\kappa$-Minkowski space, Nucl. Phys. B, Proc. Suppl. 102, 161 (2001).

[13] P. Kosinski, P. Maslanka, J. Lukierski, and A. Sitarz, Generalized $\kappa$ deformations and deformed relativistic scalar fields on noncommutative Minkowski space, arXiv:hep-th/ 0307038 .

[14] A. Agostini, F. Lizzi, and A. Zampini, Generalized Weyl systems and kappa Minkowski space, Mod. Phys. Lett. A 17, 2105 (2002).

[15] M. Dimitrijevic, L. Jonke, L. Moller, E. Tsouchnika, J. Wess, and M. Wohlgenannt, Deformed field theory on $\kappa$ space-time, Eur. Phys. J. C 31, 129 (2003).

[16] A. Agostini, G. Amelino-Camelia, and F. d'Andrea, Hopfalgebra description of noncommutative-space-time symmetries, Int. J. Mod. Phys. A 19, 5187 (2004).

[17] A. Agostini, G. Amelino-Camelia, and M. Arzano, Dirac spinors for doubly special relativity and $\kappa$ Minkowski noncummutative space-time, Classical Quantum Gravity 21, 2179 (2004).

[18] M. Dimitrijevic, L. Jonke, and L. Moller, U(1) gauge field theory on $\kappa$-Minkowski space, J. High Energy Phys. 09 (2005) 068.

[19] A. Agostini, G. Amelino-Camelia, M. Arzano, and F. D'Andrea, A cyclic integral on $\kappa$-Minkowski noncommutative space-time, Int. J. Mod. Phys. A 21, 3133 (2006).

[20] A. Agostini, G. Amelino-Camelia, M. Arzano, A. Marciano, and R. A. Tacchi, Generalizing the Noether theorem for Hopf-algebra spacetime symmetries, Mod. Phys. Lett. A 22, 1779 (2007).
[21] M. Arzano, Quantum fields, Noether charges and $\kappa$-spacetime symmetries, Proc. Sci. QG-PH2007 (2007) 005.

[22] M. Arzano and A. Marciano, Fock space, quantum fields, and $\kappa$-Poincaré symmetries, Phys. Rev. D 76, 125005 (2007).

[23] M. Arzano and A. Marciano, Symplectic geometry and Noether charges for Hopf algebra space-time symmetries, Phys. Rev. D 75, 081701 (2007).

[24] L. Freidel, J. Kowalski-Glikman, and S. Nowak, Field theory on $\kappa$-Minkowski space revisited: Noether charges and breaking of Lorentz symmetry, Int. J. Mod. Phys. A 23, 2687 (2008).

[25] G. Amelino-Camelia, G. Gubitosi, A. Marciano, P. Martinetti, and F. Mercati, A no-pure-boost uncertainty principle from spacetime noncommutativity, Phys. Lett. B 671, 298 (2009).

[26] M. Arzano, J. Kowalski-Glikman, and A. Walkus, Lorentz invariant field theory on $\kappa$-Minkowski space, Classical Quantum Gravity 27, 025012 (2010).

[27] M. Arzano and D. Benedetti, Rainbow statistics, Int. J. Mod. Phys. A 24, 4623 (2009).

[28] M. Dimitrijevic and L. Jonke, A Twisted look on $\kappa$-Minkowski: U(1) gauge theory, J. High Energy Phys. 12 (2011) 080.

[29] M. Arzano, Anatomy of a deformed symmetry: Field quantization on curved momentum space, Phys. Rev. D 83, 025025 (2011).

[30] G. Gubitosi and F. Mercati, Relative locality in $\kappa$-Poincaré, Classical Quantum Gravity 30, 145002 (2013).

[31] J. Kowalski-Glikman and S. Nowak, Doubly special relativity and de Sitter space, Classical Quantum Gravity 20, 4799 (2003).

[32] G. Amelino-Camelia, Relativity in spacetimes with short-distance structure governed by an observerindependent (Planckian) length scale, Int. J. Mod. Phys. D 11, 35 (2002).

[33] J. Magueijo and L. Smolin, Lorentz Invariance with an Invariant Energy Scale, Phys. Rev. Lett. 88, 190403 (2002).

[34] J. Magueijo and L. Smolin, Generalized Lorentz invariance with an invariant energy scale, Phys. Rev. D 67, 044017 (2003).

[35] G. Amelino-Camelia, Quantum-spacetime phenomenology, Living Rev. Relativ. 16, 5 (2013).

[36] S. Liberati, S. Sonego, and M. Visser, Interpreting doubly special relativity as a modified theory of measurement, Phys. Rev. D 71, 045001 (2005).

[37] S. Judes and M. Visser, Conservation laws in "doubly special relativity", Phys. Rev. D 68, 045001 (2003).

[38] G. Amelino-Camelia and S. Majid, Waves on noncommutative space-time and gamma-ray bursts, Int. J. Mod. Phys. A 15, 4301 (2000).

[39] V. A. Kostelecký and J. D. Tasson, Prospects for Large Relativity Violations in Matter-Gravity Couplings, Phys. Rev. Lett. 102, 010402 (2009).

[40] J. R. Ellis, N. E. Mavromatos, D. V. Nanopoulos, A. S. Sakharov, and E. K. G. Sarkisyan, Robust limits on Lorentz violation from gamma-ray bursts, Astropart. Phys. 25, 402 (2006); Erratum, Astropart. Phys.29, 158(E) (2008). 
[41] J. R. Ellis, K. Farakos, N. E. Mavromatos, V. A. Mitsou, and D. V. Nanopoulos, Astrophysical probes of the constancy of the velocity of light, Astrophys. J. 535, 139 (2000).

[42] J. R. Ellis, N. E. Mavromatos, and A. S. Sakharov, Synchrotron radiation from the Crab Nebula discriminates between models of space-time foam, Astropart. Phys. 20, 669 (2004).

[43] G. Amelino-Camelia, J. R. Ellis, N. E. Mavromatos, D. V. Nanopoulos, and S. Sarkar, Potential sensitivity of $\gamma$-ray burster observations to wave dispersion in vacuo, Nature (London) 393, 763 (1998).

[44] A. A. Abdo et al., A limit on the variation of the speed of light arising from quantum gravity effects, Nature (London) 462, 331 (2009).

[45] G. Amelino-Camelia, L. Barcaroli, G. D’Amico, N. Loret, and $\mathrm{G}$. Rosati, IceCube and GRB neutrinos propagating in quantum spacetime, Phys. Lett. B 761, 318 (2016).

[46] J. Ellis, N.E. Mavromatos, and D. V. Nanopoulos, Comments on graviton propagation in light of GW150914, Mod. Phys. Lett. A 31, 1675001 (2016).

[47] G. Amelino-Camelia, M. Arzano, G. Gubitosi, and J. Magueijo, Rainbow gravity and scale-invariant fluctuations, Phys. Rev. D 88, 041303 (2013).

[48] J. R. Ellis, N. E. Mavromatos, and D. V. Nanopoulos, A microscopic recoil model for light cone fluctuations in quantum gravity, Phys. Rev. D 61, 027503 (1999).

[49] G. Amelino-Camelia, J. R. Ellis, N. E. Mavromatos, and D. V. Nanopoulos, Distance measurement and wave dispersion in a Liouville string approach to quantum gravity, Int. J. Mod. Phys. A 12, 607 (1997).

[50] J. R. Ellis, N. E. Mavromatos, and D. V. Nanopoulos, Derivation of a vacuum refractive index in a stringy space-time foam model, Phys. Lett. B 665, 412 (2008).

[51] V. A. Kostelecky and S. Samuel, Spontaneous breaking of Lorentz symmetry in string theory, Phys. Rev. D 39, 683 (1989).

[52] J. Magueijo and L. Smolin, String theories with deformed energy momentum relations, and a possible nontachyonic bosonic string, Phys. Rev. D 71, 026010 (2005).

[53] R. Gambini and J. Pullin, Nonstandard optics from quantum space-time, Phys. Rev. D 59, 124021 (1999).

[54] J. Alfaro, M. Reyes, H. A. Morales-Tecotl, and L. F. Urrutia, On alternative approaches to Lorentz violation invariance in loop quantum gravity inspired models, Phys. Rev. D 70, 084002 (2004).

[55] V. Chari and A. N. Pressley, A Guide to Quantum Groups (Cambridge University Press, Cambridge, England, 1995).

[56] A. Ballesteros, C. Meusburger, and P. Naranjo, AdS poisson homogeneous spaces and Drinfel'd doubles, J. Phys. A 50, 395202 (2017).
[57] F. Mercati, A perspective on the theory and phenomenology of quantum spacetime, Ph. D. thesis, Sapienza University of Rome, 2010, http://www.phys.uniroma1.it/fisica/sites/ default/files/DOTT_FISICA/MENU/03DOTTORANDI/ TesiFin23/Mercati.pdf.

[58] J. Kowalski-Glikman, A short introduction to $\kappa$-deformation, Int. J. Mod. Phys. A 32, 1730026 (2017).

[59] L. Freidel, J. Kowalski-Glikman, and S. Nowak, From noncommutative $\kappa$-Minkowski to Minkowski space-time, Phys. Lett. B 648, 70 (2007).

[60] G. Amelino-Camelia, G. Gubitosi, and F. Mercati, Discreteness of area in noncommutative space, Phys. Lett. B 676, 180 (2009).

[61] C. A. S. Young and R. Zegers, Covariant particle statistics and intertwiners of the $\kappa$-deformed Poincare algebra, Nucl. Phys. B797, 537 (2008).

[62] M. Daszkiewicz, J. Lukierski, and M. Woronowicz, Towards quantum noncommutative $\kappa$-deformed field theory, Phys. Rev. D 77, 105007 (2008).

[63] M. Daszkiewicz, J. Lukierski, and M. Woronowicz, $\kappa$-deformed oscillators, the choice of star product and free kappa-deformed quantum fields, J. Phys. A 42, 355201 (2009).

[64] T. R. Govindarajan, K. S. Gupta, E. Harikumar, S. Meljanac, and D. Meljanac, Twisted statistics in $\kappa$-Minkowski spacetime, Phys. Rev. D 77, 105010 (2008).

[65] M. Arzano, J. Kowalski-Glikman, and T. Trześniewski, Beyond Fock space in three dimensional semiclassical gravity, Classical Quantum Gravity 31, 035013 (2014).

[66] L. Dabrowski and G. Piacitelli, Canonical $k$-Minkowski spacetime, arXiv:1004.5091.

[67] G. Amelino-Camelia, N. Loret, and G. Rosati, Speed of particles and a relativity of locality in $\kappa$-Minkowski quantum spacetime, Phys. Lett. B 700, 150 (2011).

[68] S. Meljanac, D. Meljanac, F. Mercati, and D. Pikutić, Noncommutative spaces and Poincaré symmetry, Phys. Lett. B 766, 181 (2017).

[69] G. Amelino-Camelia, Limits on the measurability of space-time distances in (the semiclassical approximation of) quantum gravity, Mod. Phys. Lett. A 09, 3415 (1994).

[70] F. Mercati and M. Sergola, Light cone in a quantum spacetime (to be published).

[71] M. J. Neves, C. Farina, and M. V. Cougo-Pinto, Past and Future Blurring at Fundamental Length Scale, Phys. Rev. Lett. 105, 211601 (2010).

[72] N. Bruno, G. Amelino-Camelia, and J. Kowalski-Glikman, Deformed boost transformations that saturate at the Planck scale, Phys. Lett. B 522, 133 (2001). 